\title{
On a WKB-Theoretic Transformation for a Completely Integrable System near a Degenerate Point where Two Turning Points Coalesce
}

\author{
by
}

\author{
Sampei Hirose
}

\begin{abstract}
We discuss the exact WKB analysis for a completely integrable system in two variables with a large parameter. Using a WKB-theoretic transformation, we study the behavior of the system near a degenerate point where two turning points coalesce.

2010 Mathematics Subject Classification: Primary 34M60; Secondary 33C70, 34E20, $58 \mathrm{~A} 17$.

Keywords: exact WKB analysis, completely integrable system, Pearcey system.
\end{abstract}

\section{$\S 1$. Introduction}

In this paper, we study the exact WKB analysis for a completely integrable system (a holonomic system) in two variables with a large parameter $\eta>0$ :

$$
\begin{cases}\eta^{-1} \frac{\partial}{\partial x_{1}} \Psi=P\left(x_{1}, x_{2}, \eta\right) \Psi, & P\left(x_{1}, x_{2}, \eta\right)=\sum_{n \geq 0} \eta^{-n} P_{n}\left(x_{1}, x_{2}\right), \\ \eta^{-1} \frac{\partial}{\partial x_{2}} \Psi=Q\left(x_{1}, x_{2}, \eta\right) \Psi, & Q\left(x_{1}, x_{2}, \eta\right)=\sum_{n \geq 0} \eta^{-n} Q_{n}\left(x_{1}, x_{2}\right),\end{cases}
$$

where $P_{n}\left(x_{1}, x_{2}\right)$ and $Q_{n}\left(x_{1}, x_{2}\right)(n=0,1,2, \ldots)$ are $3 \times 3$ matrices with holomorphic entries.

As in the case of ordinary differential equations, the Stokes geometry (i.e., turning points and Stokes surfaces) is an important ingredient of the exact WKB analysis for completely integrable systems. In $[\mathrm{H}]$, we discussed the Stokes geometry for two concrete completely integrable systems: the Pearcey system and the

Communicated by H. Okamoto. Received December 27, 2012. Revised July 29, 2013.

S. Hirose: Research Institute for Mathematical Sciences, Kyoto University,

Kyoto 606-8502, Japan;

e-mail: mathsamp@kurims.kyoto-u.ac.jp

(C) 2014 Research Institute for Mathematical Sciences, Kyoto University. All rights reserved. 
$(1,4)$ hypergeometric system. For example, the Pearcey system is a completely integrable system of the form

$$
\left\{\begin{array}{l}
\eta^{-1} \frac{\partial}{\partial x_{1}} \Psi=P_{0}\left(x_{1}, x_{2}\right) \Psi \\
\eta^{-1} \frac{\partial}{\partial x_{2}} \Psi=\left\{Q_{0}\left(x_{1}, x_{2}\right)+\eta^{-1} Q_{1}\left(x_{1}, x_{2}\right)\right\} \Psi
\end{array}\right.
$$

where

$$
P_{0}=\left(\begin{array}{ccc}
0 & 1 & 0 \\
0 & 0 & 1 \\
-x_{1} / 4 & -x_{2} / 2 & 0
\end{array}\right), \quad Q_{0}=P_{0}^{2}+\frac{x_{2}}{3}, \quad Q_{1}=\frac{\partial P_{0}}{\partial x_{1}}
$$

This system is obtained from the system of partial differential equations

$$
\left\{\begin{array}{l}
\left(\frac{\partial^{3}}{\partial x_{1}^{3}}+\frac{x_{2}}{2} \eta^{2} \frac{\partial}{\partial x_{1}}+\frac{x_{1}}{4} \eta^{3}\right) \psi=0 \\
\left(\eta \frac{\partial}{\partial x_{2}}-\frac{\partial^{2}}{\partial x_{1}^{2}}\right) \psi=0
\end{array}\right.
$$

satisfied by the Pearcey integral $([\mathrm{P}])$

$$
\psi=\int \exp \left\{\eta\left(t^{4}+x_{2} t^{2}+x_{1} t\right)\right\} d t
$$

through the transformation

$$
\Psi=\exp \left(\eta \frac{x_{2}^{2}}{6}\right)\left(\begin{array}{c}
\psi \\
\eta^{-1} \frac{\partial}{\partial x_{1}} \psi \\
\eta^{-2} \frac{\partial^{2}}{\partial x_{1}^{2}} \psi
\end{array}\right)
$$

As discussed in $[\mathrm{H}]$, the point $\left(x_{1}, x_{2}\right)=(0,0)$ plays a crucial role in the global study of the Stokes geometry for the Pearcey system (1.2)-(1.3). In fact, it is a degenerate point where the characteristic equation

$$
\xi_{1}^{3}+\frac{x_{2}}{2} \xi_{1}+\frac{x_{1}}{4}=0
$$

of the first equation

$$
\left(\frac{\partial^{3}}{\partial x_{1}^{3}}+\frac{x_{2}}{2} \eta^{2} \frac{\partial}{\partial x_{1}}+\frac{x_{1}}{4} \eta^{3}\right) \psi=0
$$

of (1.4) has a triple root. Furthermore, at $\left(x_{1}, x_{2}\right)=(0,0)$ we observe a phenomenon that two turning points $x_{1}=\tau^{(1)}\left(x_{2}\right)$ and $x_{1}=\tau^{(2)}\left(x_{2}\right)$ of (1.8) of different types for $x_{2} \neq 0$ (i.e., $x_{1}=\tau^{(i)}\left(x_{2}\right)(i=1,2)$ are points where $(1.7)$ 
has a double root and, further, their types are different in the sense that pairs of roots merging at $x_{1}=\tau^{(i)}\left(x_{2}\right)$ are different while sharing only one common root) coalesce at $x_{1}=0$ when $x_{2}$ tends to 0 . This degeneracy is related to the fact that a new Stokes curve of (1.8) discussed by Berk et al. [BNR] is included in the Stokes surface for the Pearcey system and plays a central role in the global study of the Stokes geometry for the Pearcey system (1.2)-(1.3).

As shown in $[\mathrm{H}]$, similar results also hold for the $(1,4)$ hypergeometric system. Thus, in this paper, we study the behavior of a general completely integrable system (1.1) in two variables with $3 \times 3$ matrix coefficients near a degenerate point where two turning points of different types coalesce.

The main theorem of this paper is as follows: We consider a completely integrable system $(1.1)$ near $\left(x_{1}, x_{2}\right)=(0,0)$, where two turning points of different types coalesce. Here we assume that $P_{n}\left(x_{1}, x_{2}\right)$ and $Q_{n}\left(x_{1}, x_{2}\right)(n=0,1,2, \ldots)$ are $3 \times 3$ matrices with holomorphic entries in $D_{\rho_{0}}=\left\{\left(x_{1}, x_{2}\right) \in \mathbb{C}^{2} ;\left|x_{j}\right| \leq \rho_{0}\right\}$ satisfying

$$
\left\|P_{n}\right\|_{\rho_{0}, \rho_{0}},\left\|Q_{n}\right\|_{\rho_{0}, \rho_{0}} \leq C \alpha^{n} n !
$$

with some norm $\|\cdot\|_{\rho_{0}, \rho_{0}}$ (see (2.3), (2.4) for the precise definition) and some positive constants $C$ and $\alpha$. As we will see later (cf. (2.8)), we may assume $\operatorname{tr} P_{0}=$ $\operatorname{tr} Q_{0}=0$ without loss of generality. Then, letting $a_{2}(x)$ and $a_{3}(x)$ be holomorphic functions defined by $\operatorname{det}\left(\xi_{1}-P_{0}(x)\right)=\xi_{1}^{3}+a_{2}(x) \xi_{1}+a_{3}(x)$, we may assume that $a_{2}(x)$ and $a_{3}(x)$ satisfy

(i) $a_{2}(0)=a_{3}(0)=0$,

since the characteristic equation $\xi_{1}^{3}+a_{2}(x) \xi_{1}+a_{3}(x)=0$ is expected to have a triple root at a degenerate point where two turning points of different types coalesce. In addition, we suppose

(ii) $\frac{\partial a_{3}}{\partial x_{1}}(0) \neq 0$.

(iii) $\operatorname{det}\left(\begin{array}{ll}\frac{\partial a_{2}}{\partial x_{1}}(0) & \frac{\partial a_{2}}{\partial x_{2}}(0) \\ \frac{\partial a_{3}}{\partial x_{1}}(0) & \frac{\partial a_{3}}{\partial x_{2}}(0)\end{array}\right) \neq 0$.

(In fact, under these assumptions, we can show that two turning points of different types coalesce at $\left(x_{1}, x_{2}\right)=(0,0)$.) Then there exists a WKB-theoretic transformation

$$
\begin{aligned}
\Psi\left(x_{1}, x_{2}, \eta\right) & =T\left(x_{1}, x_{2}, \eta\right) \widetilde{\Psi}\left(\widetilde{x}_{1}\left(x_{1}, x_{2}\right), \widetilde{x}_{2}\left(x_{1}, x_{2}\right), \eta\right), \\
T\left(x_{1}, x_{2}, \eta\right) & =\sum_{n \geq 0} \eta^{-n} T_{n}\left(x_{1}, x_{2}\right)
\end{aligned}
$$


near $\left(x_{1}, x_{2}\right)=(0,0)$ transforming the completely integrable system (1.1) into the Pearcey system (1.2)-(1.3). That is, if $\Psi\left(x_{1}, x_{2}, \eta\right)$ is a solution of (1.1), then $\widetilde{\Psi}\left(\widetilde{x}_{1}, \widetilde{x}_{2}, \eta\right)$ defined by $(1.10)-(1.11)$ is a solution of the Pearcey system (1.2)(1.3) with the independent variable $\left(\widetilde{x}_{1}, \widetilde{x}_{2}\right)$. Here $\left(\widetilde{x}_{1}\left(x_{1}, x_{2}\right), \widetilde{x}_{2}\left(x_{1}, x_{2}\right)\right)$ is a biholomorphic coordinate transformation near $\left(x_{1}, x_{2}\right)=(0,0)$ and $T_{n}\left(x_{1}, x_{2}\right)$ $(n=0,1,2, \ldots)$ are $3 \times 3$ matrices with holomorphic entries in $D_{\rho}$ for some $0<\rho<\rho_{0}$ satisfying

$$
\left\|T_{n}\right\|_{\rho, \rho} \leq \widetilde{C} \widetilde{\alpha}^{n} n !
$$

with some positive constants $\widetilde{C}$ and $\widetilde{\alpha}$.

We explain the meaning of the assumptions (ii) and (iii). First, (ii) (together with (i)) means that $P_{0}$ and $Q_{0}$ satisfy a relation similar to the second relation of (1.3) near $\left(x_{1}, x_{2}\right)=(0,0)$. To be more specific, there exist holomorphic functions $b_{k}\left(x_{1}, x_{2}\right)(k=0,1,2)$ near $\left(x_{1}, x_{2}\right)=(0,0)$ such that

$$
Q_{0}=b_{2}\left(x_{1}, x_{2}\right) P_{0}^{2}+b_{1}\left(x_{1}, x_{2}\right) P_{0}+b_{0}\left(x_{1}, x_{2}\right)
$$

(cf. Lemma 2.4). Furthermore, by using this relation we can verify that the set of turning points for the completely integrable system (1.1) is given by the zeros of the discriminant (with respect to $\xi_{1}$ ) of the characteristic equation $\operatorname{det}\left(\xi_{1}-P_{0}\left(x_{1}, x_{2}\right)\right.$ ) of $P_{0}\left(x_{1}, x_{2}\right)$ (cf. Proposition 2.6). We can also confirm that (ii) automatically follows from (i) and (iii) by using the compatibility condition (see Proposition 2.9). Next, (iii) means that the set of turning points for (1.1) is analytically equivalent to the set of turning points for the Pearcey system $\left\{27 x_{1}^{2}+8 x_{2}^{3}=0\right\}$ near $\left(x_{1}, x_{2}\right)=(0,0)$. The claim of the main theorem is that, under these geometric assumptions, the completely integrable system (1.1) is transformed into the Pearcey system (1.2)-(1.3) by a WKB-theoretic transformation near a degenerate point where two turning points of different types coalesce. In particular, near such a point, the Stokes geometry for (1.1) is analytically equivalent to the Stokes geometry for the Pearcey system (1.2)-(1.3) by the coordinate transformation $\left(\widetilde{x}_{1}\left(x_{1}, x_{2}\right), \widetilde{x}_{2}\left(x_{1}, x_{2}\right)\right)$ in the main theorem.

The WKB-theoretic transformation (1.10)-(1.11) is, as a formal transformation, the same as the one that Wasow [W1], [W2] used to transform a system of ordinary differential equations

$$
\eta^{-1} \frac{d}{d x} \Psi=P(x, \eta) \Psi, \quad P(x, \eta)=\sum_{n \geq 0} \eta^{-n} P_{n}(x)
$$

into a canonical form. Aoki-Kawai-Takei $[\mathrm{AKT}]$ first used this type of transformation in the framework of the exact WKB analysis, that is, in connection with the Borel resummation method with respect to the large parameter $\eta$. In $[\mathrm{AKT}]$, 
they considered a WKB-theoretic transformation for a second-order linear ordinary differential equation

$$
\left(\frac{d^{2}}{d x^{2}}-\eta^{2} Q(x)\right) \psi=0
$$

near a turning point and showed that (1.15) can be transformed into the Airy equation near a simple turning point by a WKB-theoretic transformation, the transformation acts analytically on Borel transformed WKB solutions, and several properties (for example, the connection formula on Stokes curves) of the Borel sum of WKB solutions can be obtained from these facts. In this paper, motivated by [T], we employ the WKB-theoretic transformation (1.10)-(1.11) for the analysis of a completely integrable system (1.1) in two variables. In particular, the estimate (1.12) guarantees that $T\left(x_{1}, x_{2}, \eta\right)$ acts analytically on the Borel transform of a WKB solution $\widetilde{\Psi}$.

The paper is organized as follows: In Section 2, we explain the precise statement of the main theorem and discuss a generalization of the main theorem to completely integrable systems in two variables with $m \times m$ matrix coefficients. Furthermore, we give some remarks on the main theorem in Subsection 2.1. In particular, we show an important property of the set of turning points and the Stokes surface for (1.1). The lemmas proved in Subsection 2.1 play an important role throughout the paper. In Subsection 2.2, we give a proof of the generalization of the main theorem to completely integrable systems with $m \times m$ matrix coefficients by using the main theorem. Then in Section 3 we consider some examples to which the main theorem is applicable. We discuss the $(1,4)$ hypergeometric system in Subsection 3.1 and the $(2,3)$ hypergeometric system in Subsection 3.2. In Sections 4 and 5 , we give a proof of the main theorem. We construct a WKB-theoretic transformation in Section 4 and prove the estimate (1.12) for it in Section 5.

\section{§2. Main theorem}

In this section, we first consider the completely integrable system

$$
\begin{cases}\eta^{-1} \frac{\partial}{\partial x_{1}} \Psi=P(x, \eta) \Psi, & P(x, \eta)=\sum_{n \geq 0} \eta^{-n} P_{n}(x), \\ \eta^{-1} \frac{\partial}{\partial x_{2}} \Psi=Q(x, \eta) \Psi, & Q(x, \eta)=\sum_{n \geq 0} \eta^{-n} Q_{n}(x),\end{cases}
$$

where $P_{n}(x)$ and $Q_{n}(x)$ are $3 \times 3$ matrices with holomorphic entries in $D_{\rho_{0}}=\{x=$ $\left.\left(x_{1}, x_{2}\right) \in \mathbb{C}^{2} ;\left|x_{j}\right| \leq \rho_{0}\right\}$ for some $\rho_{0}>0$ and satisfy an estimate

$$
\left\|P_{n}\right\|_{\rho_{0}, \rho_{0}},\left\|Q_{n}\right\|_{\rho_{0}, \rho_{0}} \leq C \alpha^{n} n ! \quad(n \geq 0)
$$


with some positive constants $C$ and $\alpha$. Here for a matrix $A(x)=\left(a_{i j}(x)\right)$ with holomorphic entries in $D_{\rho_{0}}\left(0<\rho_{1}, \rho_{2}<\rho_{0}\right)$ we define

$$
\begin{aligned}
\|A\|_{\rho_{1}, \rho_{2}} & =\sup _{\left|x_{1}\right| \leq \rho_{1},\left|x_{2}\right| \leq \rho_{2}}\|A(x)\|, \\
\|A(x)\| & =\sum_{i, j}\left|a_{i j}(x)\right| .
\end{aligned}
$$

Note that the system satisfies the compatibility condition

$$
[P, Q]+\eta^{-1} \frac{\partial P}{\partial x_{2}}-\eta^{-1} \frac{\partial Q}{\partial x_{1}}=0
$$

As a consequence we have

$$
\begin{gathered}
{\left[P_{0}, Q_{0}\right]=0} \\
{\left[P_{1}, Q_{0}\right]+\left[P_{0}, Q_{1}\right]+\frac{\partial P_{0}}{\partial x_{2}}-\frac{\partial Q_{0}}{\partial x_{1}}=0 .}
\end{gathered}
$$

Without loss of generality we may assume that

$$
\operatorname{tr} P_{0}=\operatorname{tr} Q_{0}=0 .
$$

In fact, taking the trace of (2.5), we obtain

$$
\operatorname{tr}\left(\frac{\partial P}{\partial x_{2}}-\frac{\partial Q}{\partial x_{1}}\right)=0
$$

In particular, $\operatorname{tr}\left(P_{0}(x) d x_{1}+Q_{0}(x) d x_{2}\right)$ is a closed one-form. Then, by a gauge transformation $\Psi=\exp \left\{\eta \int^{x} \operatorname{tr}\left(P_{0}(x) d x_{1}+Q_{0}(x) d x_{2}\right) / 3\right\} \Phi,(2.1)$ is transformed into

$$
\left\{\begin{array}{l}
\eta^{-1} \frac{\partial}{\partial x_{1}} \Phi=\left(P(x, \eta)-\frac{\operatorname{tr} P_{0}(x)}{3}\right) \Phi \\
\eta^{-1} \frac{\partial}{\partial x_{2}} \Phi=\left(Q(x, \eta)-\frac{\operatorname{tr} Q_{0}(x)}{3}\right) \Phi .
\end{array}\right.
$$

Our main theorem is the following

Theorem 2.1. Let $a_{2}(x)$ and $a_{3}(x)$ be holomorphic functions defined by the relation $\operatorname{det}\left(\xi_{1}-P_{0}(x)\right)=\xi_{1}^{3}+a_{2}(x) \xi_{1}+a_{3}(x)$. Suppose that

(i) $a_{2}(0)=a_{3}(0)=0$.

(ii) $\frac{\partial a_{3}}{\partial x_{1}}(0) \neq 0$.

(iii) $\operatorname{det}\left(\begin{array}{ll}\frac{\partial a_{2}}{\partial x_{1}}(0) & \frac{\partial a_{2}}{\partial x_{2}}(0) \\ \frac{\partial a_{3}}{\partial x_{1}}(0) & \frac{\partial a_{3}}{\partial x_{2}}(0)\end{array}\right) \neq 0$. 
Then there exist a sufficiently small positive constant $0<\rho<\rho_{0}$, holomorphic functions $\widetilde{x}_{i}(x)(i=1,2)$ in $D_{\rho}$, and an infinite sequence $\left\{T_{n}(x)\right\}_{n \geq 0}$ of $3 \times 3$ matrices with the following properties:

- $\widetilde{x}_{1}(0)=\widetilde{x}_{2}(0)=0$.

- $\widetilde{x}(x)=\left(\widetilde{x}_{1}(x), \widetilde{x}_{2}(x)\right)$ is a biholomorphic map from $D_{\rho}$ to $\widetilde{x}\left(D_{\rho}\right)$.

- Every entry of $T_{n}(x)$ is holomorphic in $D_{\rho}$ and $\operatorname{det} T_{0}(x) \neq 0\left(x \in D_{\rho}\right)$.

- $T_{n}(x)(n \geq 0)$ satisfy

$$
\left\|T_{n}\right\|_{\rho, \rho} \leq \widetilde{C} \widetilde{\alpha}^{n} n !
$$

with some positive constants $\widetilde{C}$ and $\widetilde{\alpha}$.

- The formal transformation

$$
\Psi(x, \eta)=T(x, \eta) \widetilde{\Psi}(\widetilde{x}(x), \eta), \quad T(x, \eta)=\sum_{n \geq 0} \eta^{-n} T_{n}(x),
$$

transforms (2.1) into the system

$$
\left\{\begin{array}{l}
\eta^{-1} \frac{\partial}{\partial \widetilde{x}_{1}} \widetilde{\Psi}=\widetilde{P}(\widetilde{x}, \eta) \widetilde{\Psi}, \quad \widetilde{P}(\widetilde{x}, \eta)=\sum_{n \geq 0} \eta^{-n} \widetilde{P}_{n}(\widetilde{x}), \\
\eta^{-1} \frac{\partial}{\partial \widetilde{x}_{2}} \widetilde{\Psi}=\widetilde{Q}(\widetilde{x}, \eta) \widetilde{\Psi}, \quad \widetilde{Q}(\widetilde{x}, \eta)=\sum_{n \geq 0} \eta^{-n} \widetilde{Q}_{n}(\widetilde{x}),
\end{array}\right.
$$

where $\widetilde{P}_{n}(\widetilde{x})$ and $\widetilde{Q}_{n}(\widetilde{x})$ are given as follows:

$$
\begin{aligned}
& \widetilde{P}_{0}=\left(\begin{array}{ccc}
0 & 1 & 0 \\
0 & 0 & 1 \\
-\widetilde{x}_{1} / 4 & -\widetilde{x}_{2} / 2 & 0
\end{array}\right), \quad \widetilde{P}_{n}=0 \quad(n \geq 1), \\
& \widetilde{Q}_{0}=\widetilde{P}_{0}^{2}+\frac{\widetilde{x}_{2}}{3}, \quad \widetilde{Q}_{1}=\frac{\partial \widetilde{P}_{0}}{\partial \widetilde{x}_{1}}, \quad \widetilde{Q}_{n}=0 \quad(n \geq 2) .
\end{aligned}
$$

Note that (2.13) is equivalent to the Pearcey system

$$
\left\{\begin{array}{l}
\left(\frac{\partial^{3}}{\partial \widetilde{x}_{1}^{3}}+\frac{\widetilde{x}_{2}}{2} \eta^{2} \frac{\partial}{\partial \widetilde{x}_{1}}+\frac{\widetilde{x}_{1}}{4} \eta^{3}\right) \widetilde{\psi}=0 \\
\left(\eta \frac{\partial}{\partial \widetilde{x}_{2}}-\frac{\partial^{2}}{\partial \widetilde{x}_{1}^{2}}\right) \widetilde{\psi}=0
\end{array}\right.
$$

through the transformation

$$
\widetilde{\Psi}=\exp \left(\eta \frac{\widetilde{x}_{2}^{2}}{6}\right)\left(\begin{array}{c}
\widetilde{\psi} \\
\eta^{-1} \frac{\partial}{\partial \widetilde{x}_{1}} \widetilde{\psi} \\
\eta^{-2} \frac{\partial^{2}}{\partial \widetilde{x}_{1}^{2}} \widetilde{\psi}
\end{array}\right)
$$

We also call (2.13) the Pearcey system in this paper. 
We next consider a generalization of Theorem 2.1 to a completely integrable system in two variables with $m \times m$ matrix coefficients:

$$
\begin{cases}\eta^{-1} \frac{\partial}{\partial x_{1}} \Psi=P(x, \eta) \Psi, & P(x, \eta)=\sum_{n \geq 0} \eta^{-n} P_{n}(x), \\ \eta^{-1} \frac{\partial}{\partial x_{2}} \Psi=Q(x, \eta) \Psi, & Q(x, \eta)=\sum_{n \geq 0} \eta^{-n} Q_{n}(x),\end{cases}
$$

where $P_{n}(x)$ and $Q_{n}(x)$ are $m \times m$ matrices with holomorphic entries in $D_{\rho_{0}}=$ $\left\{x=\left(x_{1}, x_{2}\right) \in \mathbb{C}^{2} ;\left|x_{j}\right| \leq \rho_{0}\right\}$ for some $\rho_{0}>0$ and satisfy

$$
\left\|P_{n}\right\|_{\rho_{0}, \rho_{0}},\left\|Q_{n}\right\|_{\rho_{0}, \rho_{0}} \leq C \alpha^{n} n ! \quad(n \geq 0)
$$

with some positive constants $C$ and $\alpha$.

Theorem 2.2. Suppose that $D\left(x, \xi_{1}\right)=\operatorname{det}\left(\xi_{1}-P_{0}(x)\right)$ satisfies the following conditions:

${\left(\mathrm{i}^{\star}\right)}^{\star}$ The equation $D\left(0, \xi_{1}\right)=0$ has a triple root $\xi_{1}^{\star}$, that is,

$$
D\left(0, \xi_{1}^{\star}\right)=\frac{\partial D}{\partial \xi_{1}}\left(0, \xi_{1}^{\star}\right)=\frac{\partial^{2} D}{\partial \xi_{1}^{2}}\left(0, \xi_{1}^{\star}\right)=0, \quad \frac{\partial^{3} D}{\partial \xi_{1}^{3}}\left(0, \xi_{1}^{\star}\right) \neq 0 .
$$

$\left(\mathrm{ii}^{\star}\right) \frac{\partial D}{\partial x_{1}}\left(0, \xi_{1}^{\star}\right) \neq 0$.

$\left.\left(\right.$ iii $\left.^{\star}\right) \operatorname{det}\left(\begin{array}{cc}\frac{\partial D}{\partial x_{1}} & \frac{\partial D}{\partial x_{2}} \\ \frac{\partial^{2} D}{\partial x_{1} \partial \xi_{1}} & \frac{\partial^{2} D}{\partial x_{2} \partial \xi_{1}}\end{array}\right)\right|_{\left(x, \xi_{1}\right)=\left(0, \xi_{1}^{\star}\right)} \neq 0$.

Then there exist a sufficiently small positive constant $0<\rho<\rho_{0}$ and an infinite sequence $\left\{T_{n}^{\star}(x)\right\}_{n \geq 0}$ of $m \times m$ matrices with the following properties:

- Every entry $T_{n}^{\star}(x)$ is holomorphic in $D_{\rho}$ and $\operatorname{det} T_{0}^{\star}(x) \neq 0\left(x \in D_{\rho}\right)$.

- $T_{n}^{\star}(x)(n \geq 0)$ satisfy

$$
\left\|T_{n}^{\star}\right\|_{\rho, \rho} \leq C^{\star}\left(\alpha^{\star}\right)^{n} n !
$$

with some positive constants $C^{\star}$ and $\alpha^{\star}$.

- The formal transformation

$$
\Psi=T^{\star}(x, \eta) \Psi^{\star}, \quad T^{\star}(x, \eta)=\sum_{n \geq 0} \eta^{-n} T_{n}^{\star}(x),
$$

transforms (2.18) into the system 


$$
\begin{cases}\eta^{-1} \frac{\partial}{\partial x_{1}} \Psi^{\star}=P^{\star}(x, \eta) \Psi^{\star}, & P^{\star}(x, \eta)=\sum_{n \geq 0} \eta^{-n} P_{n}^{\star}(x), \\ \eta^{-1} \frac{\partial}{\partial x_{2}} \Psi^{\star}=Q^{\star}(x, \eta) \Psi^{\star}, & Q^{\star}(x, \eta)=\sum_{n \geq 0} \eta^{-n} Q_{n}^{\star}(x),\end{cases}
$$

where $P_{n}^{\star}(x)$ and $Q_{n}^{\star}(x)$ are block-diagonal matrices,

$$
P_{n}^{\star}(x)=\left(\begin{array}{cc}
P_{n}^{\star(1)}(x) & \\
& P_{n}^{\star(2)}(x)
\end{array}\right), \quad Q_{n}^{\star}(x)=\left(\begin{array}{cc}
Q_{n}^{\star(1)}(x) & \\
& Q_{n}^{\star(2)}(x)
\end{array}\right),
$$

$P_{n}^{\star(1)}(x), Q_{n}^{\star(1)}(x)\left(\right.$ resp., $\left.P_{n}^{\star(2)}(x), Q_{n}^{\star(2)}(x)\right)$ are $3 \times 3$ matrices (resp., $(m-3) \times$ $(m-3)$ matrices $)$, and all coefficients $P_{n}^{\star(j)}(x)$ and $Q_{n}^{\star(j)}(x)(j=1,2, n=$ $0,1,2, \ldots)$ are holomorphic in $D_{\rho}$ and satisfy

$$
\left\|P_{n}^{\star(j)}\right\|_{\rho, \rho},\left\|Q_{n}^{\star(j)}\right\|_{\rho, \rho} \leq \widetilde{C}^{\star}\left(\widetilde{\alpha}^{\star}\right)^{n} n ! \quad(j=1,2)
$$

with some positive constants $\widetilde{C}^{\star}$ and $\widetilde{\alpha}^{\star}$. Furthermore

$$
\left.\operatorname{det}\left(\xi_{1}-P_{0}^{\star(1)}(x)\right)\right|_{\left(x, \xi_{1}\right)=\left(0, \xi_{1}^{\star}\right)}=0,\left.\quad \operatorname{det}\left(\xi_{1}-P_{0}^{\star(2)}(x)\right)\right|_{\left(x, \xi_{1}\right)=\left(0, \xi_{1}^{\star}\right)} \neq 0 .
$$

- The subsystem

$$
\begin{cases}\eta^{-1} \frac{\partial}{\partial x_{1}} \Psi^{\star(1)}=P^{\star(1)}(x, \eta) \Psi^{\star(1)}, & P^{\star(1)}(x, \eta)=\sum_{n \geq 0} \eta^{-n} P_{n}^{\star(1)}(x), \\ \eta^{-1} \frac{\partial}{\partial x_{2}} \Psi^{\star(1)}=Q^{\star(1)}(x, \eta) \Psi^{\star(1)}, & Q^{\star(1)}(x, \eta)=\sum_{n \geq 0} \eta^{-n} Q_{n}^{\star(1)}(x),\end{cases}
$$

of (2.23) can be transformed into the Pearcey system (2.13) near $x=0$ in the sense of Theorem 2.1 .

\section{§2.1. Some remarks on Theorem 2.1}

In this subsection, we make some remarks on Theorem 2.1. Let us begin with the following lemmas, which play an important role throughout this paper.

Lemma 2.3. Let $a_{2}(x)$ and $a_{3}(x)$ be holomorphic functions defined by the condition $\operatorname{det}\left(\xi_{1}-P_{0}(x)\right)=\xi_{1}^{3}+a_{2}(x) \xi_{1}+a_{3}(x)$. Suppose that:

(i) $a_{2}(0)=a_{3}(0)=0$.

(ii) $\frac{\partial a_{3}}{\partial x_{1}}(0) \neq 0$.

Then there exists a $3 \times 3$ matrix $T^{\dagger}(x)$ with the following properties:

- Every entry of $T^{\dagger}(x)$ is holomorphic near $x=0$ and $\operatorname{det} T^{\dagger}(0) \neq 0$. 
- $\left(T^{\dagger}(x)\right)^{-1} P_{0}(x) T^{\dagger}(x)=\left(\begin{array}{ccc}0 & 1 & 0 \\ 0 & 0 & 1 \\ -a_{3}(x) & -a_{2}(x) & 0\end{array}\right)$.

Proof. Since $P_{0}(0)^{3}=0$ by the assumption, there exists a constant matrix $T_{1}^{\dagger} \in$ $\mathrm{GL}(3 ; \mathbb{C})$ such that $\left(T_{1}^{\dagger}\right)^{-1} P_{0}(0) T_{1}^{\dagger}$ is one of the following:

Case 1: $\left(\begin{array}{ccc}0 & 0 & 0 \\ 0 & 0 & 0 \\ 0 & 0 & 0\end{array}\right), \quad$ Case $2:\left(\begin{array}{ccc}0 & 1 & 0 \\ 0 & 0 & 0 \\ 0 & 0 & 0\end{array}\right), \quad$ Case $3:\left(\begin{array}{ccc}0 & 1 & 0 \\ 0 & 0 & 1 \\ 0 & 0 & 0\end{array}\right)$.

Case 1. Let $p_{i}(x)(i=1,2,3)$ be $\mathbb{C}^{3}$-valued functions defined by $\left(T_{1}^{\dagger}\right)^{-1} P_{0}(x) T_{1}^{\dagger}=$ $\left(p_{1}(x), p_{2}(x), p_{3}(x)\right)$. Then $p_{i}(0)=0(i=1,2,3)$ and

$$
\begin{aligned}
\frac{\partial a_{3}}{\partial x_{1}}(0) & =-\left.\frac{\partial}{\partial x_{1}} \operatorname{det} P_{0}(x)\right|_{x=0} \\
& =-\operatorname{det}\left(\frac{\partial p_{1}}{\partial x_{1}}(0), 0,0\right)-\operatorname{det}\left(0, \frac{\partial p_{2}}{\partial x_{1}}(0), 0\right)-\operatorname{det}\left(0,0, \frac{\partial p_{3}}{\partial x_{1}}(0)\right)=0 .
\end{aligned}
$$

This contradicts the assumption.

Case 2. Let $p_{i}(x)$ be as in Case 1 . Then $p_{i}(0)=0(i=1,3)$ and

$$
\begin{aligned}
\frac{\partial a_{3}}{\partial x_{1}}(0) & =-\left.\frac{\partial}{\partial x_{1}} \operatorname{det} P_{0}(x)\right|_{x=0} \\
& =-\operatorname{det}\left(\frac{\partial p_{1}}{\partial x_{1}}(0), p_{2}(0), 0\right)-\operatorname{det}\left(0, \frac{\partial p_{2}}{\partial x_{1}}(0), 0\right)-\operatorname{det}\left(0, p_{2}(0), \frac{\partial p_{3}}{\partial x_{1}}(0)\right) \\
& =0 .
\end{aligned}
$$

This contradicts the assumption.

Hence $\left(T_{1}^{\dagger}\right)^{-1} P_{0}(0) T_{1}^{\dagger}$ must be of the form of Case 3 . We next define a $3 \times 3$ matrix $T_{2}^{\dagger}(x)$ with holomorphic entries near $x=0$ as follows:

$$
T_{2}^{\dagger}(x)=\left(\begin{array}{c}
(1,0,0) \\
(1,0,0)\left(T_{1}^{\dagger}\right)^{-1} P_{0}(x) T_{1}^{\dagger} \\
(1,0,0)\left(T_{1}^{\dagger}\right)^{-1} P_{0}(x)^{2} T_{1}^{\dagger}
\end{array}\right) .
$$

Since $\left(T_{1}^{\dagger}\right)^{-1} P_{0}(0) T_{1}^{\dagger}$ is as in Case $3, T_{2}^{\dagger}(0)$ is the identity matrix. Hence $T_{2}^{\dagger}(x)$ is invertible near $x=0$. Furthermore, we have

$$
T_{2}^{\dagger}(x)\left(T_{1}^{\dagger}\right)^{-1} P_{0}(x) T_{1}^{\dagger}=\left(\begin{array}{c}
(1,0,0)\left(T_{1}^{\dagger}\right)^{-1} P_{0}(x) T_{1}^{\dagger} \\
(1,0,0)\left(T_{1}^{\dagger}\right)^{-1} P_{0}(x)^{2} T_{1}^{\dagger} \\
(1,0,0)\left(T_{1}^{\dagger}\right)^{-1} P_{0}(x)^{3} T_{1}^{\dagger}
\end{array}\right)
$$




$$
\begin{aligned}
& =\left(\begin{array}{c}
(1,0,0)\left(T_{1}^{\dagger}\right)^{-1} P_{0}(x) T_{1}^{\dagger} \\
(1,0,0)\left(T_{1}^{\dagger}\right)^{-1} P_{0}(x)^{2} T_{1}^{\dagger} \\
-a_{3}(x)(1,0,0)-a_{2}(x)(1,0,0)\left(T_{1}^{\dagger}\right)^{-1} P_{0}(x) T_{1}^{\dagger}
\end{array}\right) \\
& =\left(\begin{array}{ccc}
0 & 1 & 0 \\
0 & 0 & 1 \\
-a_{3}(x) & -a_{2}(x) & 0
\end{array}\right) T_{2}^{\dagger}(x) .
\end{aligned}
$$

Therefore $T^{\dagger}(x)=T_{1}^{\dagger} T_{2}^{\dagger}(x)^{-1}$ has the properties of Lemma 2.3.

Using this lemma, we prove

Lemma 2.4. Under the assumptions of Lemma 2.3, there exist unique holomorphic functions $b_{k}(x)(k=0,1,2)$ near $x=0$ such that

$$
Q_{0}=b_{2}(x) P_{0}^{2}+b_{1}(x) P_{0}+b_{0}(x) .
$$

Proof. Since $\left[P_{0}, Q_{0}\right]=0$ from the compatibility condition, we have

$$
\left[\left(T^{\dagger}\right)^{-1} P_{0} T^{\dagger},\left(T^{\dagger}\right)^{-1} Q_{0} T^{\dagger}\right]=\left(T^{\dagger}\right)^{-1}\left[P_{0}, Q_{0}\right] T^{\dagger}=0,
$$

where $T^{\dagger}=T^{\dagger}(x)$ is given by Lemma 2.3 . We denote by $b_{0}(x)$ (resp., $b_{1}(x), b_{2}(x)$ ) the $(1,1)$ (resp., $(1,2),(1,3)$ ) entry of $T^{\dagger}(x)^{-1} Q_{0}(x) T^{\dagger}(x)$ and consider the matrix

$$
\left(T^{\dagger}\right)^{-1}\left(Q_{0}-b_{2}(x) P_{0}^{2}-b_{1}(x) P_{0}-b_{0}(x)\right) T^{\dagger} .
$$

For this matrix, we have

$$
\left[\left(T^{\dagger}\right)^{-1} P_{0} T^{\dagger},\left(T^{\dagger}\right)^{-1}\left(Q_{0}-b_{2}(x) P_{0}^{2}-b_{1}(x) P_{0}-b_{0}(x)\right) T^{\dagger}\right]=0,
$$

and the $(1,1),(1,2)$ and $(1,3)$ entries of $(2.30)$ are all zero. Thanks to this fact and Lemma 2.3 , the $(1,1)$ (resp., $(1,2),(1,3))$ entry of the left-hand side of $(2.31)$ is the $(2,1)$ (resp., $(2,2),(2,3))$ entry of $(2.30)$, and hence the $(2,1),(2,2)$ and $(2,3)$ entries of $(2.30)$ are all zero. By the same argument, we can verify that the $(3,1),(3,2)$ and $(3,3)$ entries of $(2.30)$ are also zero. Thus we have proved that (2.30) is the zero matrix.

We next prove the uniqueness of $b_{k}(x)(k=0,1,2)$. If holomorphic functions $\widetilde{b}_{k}(x)(k=0,1,2)$ near $x=0$ satisfy

$$
\widetilde{b}_{2}(x) P_{0}^{2}+\widetilde{b}_{1}(x) P_{0}+\widetilde{b}_{0}(x)=0,
$$

then

$$
\widetilde{b}_{2}(x)\left(\left(T^{\dagger}\right)^{-1} P_{0} T^{\dagger}\right)^{2}+\widetilde{b}_{1}(x)\left(T^{\dagger}\right)^{-1} P_{0} T^{\dagger}+\widetilde{b}_{0}(x)=0 .
$$

By the choice of $T^{\dagger}(x)$, the $(1,1),(1,2)$ and $(1,3)$ entries of the left-hand side of $(2.32)$ are $\widetilde{b}_{0}(x), \widetilde{b}_{1}(x)$ and $\widetilde{b}_{2}(x)$, respectively. Therefore $\widetilde{b}_{k}(x)=0(k=0,1,2)$. This means the uniqueness of $b_{k}(x)(k=0,1,2)$. 
Note that, by the definition of $a_{2}(x)$ and $a_{3}(x), P_{0}$ satisfies the following relation:

$$
P_{0}^{3}+a_{2}(x) P_{0}+a_{3}(x)=0
$$

Similarly, for $\widetilde{P}_{0}$ we have

$$
\widetilde{P}_{0}^{3}+\frac{\widetilde{x}_{2}}{2} \widetilde{P}_{0}+\frac{\widetilde{x}_{1}}{4}=0
$$

We now discuss the Stokes geometry for the system (2.1) near $x=0$, using Theorem 2.1. First, let us recall the definition of a turning point for the system (2.1). Let $\xi_{1, i}(i=1,2,3)$ be the three roots of the algebraic equation $\operatorname{det}\left(\xi_{1}-P_{0}(x)\right)$ $=\xi_{1}^{3}+a_{2}(x) \xi_{1}+a_{3}(x)=0$. In view of Lemma $2.4, b_{2}(x) \xi_{1, i}^{2}+b_{1}(x) \xi_{1, i}+b_{0}(x)$ is a root of the algebraic equation of $\operatorname{det}\left(\xi_{2}-Q_{0}(x)\right)=0$. Hence, we label the three roots of the algebraic equation $\operatorname{det}\left(\xi_{2}-Q_{0}(x)\right)=0$ as follows:

$$
\xi_{2, i}=b_{2}(x) \xi_{1, i}^{2}+b_{1}(x) \xi_{1, i}+b_{0}(x) \quad(i=1,2,3) .
$$

Definition 2.5. A point $c \in \mathbb{C}^{2}$ is called a turning point for the system (2.1) if there exist $i, i^{\prime} \in\{1,2,3\}\left(i \neq i^{\prime}\right)$ such that

$$
\xi_{1, i}(c)=\xi_{1, i^{\prime}}(c), \quad \xi_{2, i}(c)=\xi_{2, i^{\prime}}(c)
$$

Proposition 2.6. The set of turning points of the system (2.1) is

$$
\left\{x ; 27 a_{3}(x)^{2}+4 a_{2}(x)^{3}=0\right\} .
$$

Proof. By (2.35), the condition (2.36) is equivalent to

$$
\xi_{1, i}(c)=\xi_{1, i^{\prime}}(c) .
$$

Hence a turning point for the system (2.1) is a point where the algebraic equation $\xi_{1}^{3}+a_{2}(x) \xi_{1}+a_{3}(x)=0$ has a multiple root, that is, a zero of the discriminant of $\xi_{1}^{3}+a_{2}(x) \xi_{1}+a_{3}(x)=0$. This completes the proof.

Remark 2.7. Under the assumptions of Theorem 2.1, the set of turning points (2.37) is transformed into the set of turning points of the Pearcey system $\left\{z ; 27 z_{1}^{2}+\right.$ $\left.8 z_{2}^{3}=0\right\}$ by the coordinate transformation $z(x)=\left(4 a_{3}(x), 2 a_{2}(x)\right)$ near $x=0$. In particular, the set of turning points (2.37) has a cusp at $x=0$.

We have the following relations between $a_{k}(x)$ and $b_{k}(x)$ as a consequence of the compatibility condition. 
Lemma 2.8. Suppose that the compatibility condition (2.5) is satisfied. Then

$$
\left\{\begin{array}{l}
\frac{\partial}{\partial x_{1}}\left(2 b_{2} a_{2}-3 b_{0}\right)=0 \\
\frac{\partial a_{2}}{\partial x_{2}}=2 b_{2} \frac{\partial a_{3}}{\partial x_{1}}+b_{1} \frac{\partial a_{2}}{\partial x_{1}}+3 \frac{\partial b_{2}}{\partial x_{1}} a_{3}+2 \frac{\partial b_{1}}{\partial x_{1}} a_{2} \\
\frac{\partial a_{3}}{\partial x_{2}}=b_{1} \frac{\partial a_{3}}{\partial x_{1}}+3 \frac{\partial b_{1}}{\partial x_{1}} a_{3}-\frac{\partial b_{0}}{\partial x_{1}} a_{2} .
\end{array}\right.
$$

Proof. The transformation $\Psi=T^{\dagger}(x) \Psi^{\dagger}$ with $T^{\dagger}(x)$ given by Lemma 2.3 transforms (2.1) into

$$
\begin{cases}\eta^{-1} \frac{\partial}{\partial x_{1}} \Psi^{\dagger}=P^{\dagger}(x, \eta) \Psi^{\dagger}, & P^{\dagger}(x, \eta)=\sum_{n \geq 0} \eta^{-n} P_{n}^{\dagger}(x), \\ \eta^{-1} \frac{\partial}{\partial x_{2}} \Psi^{\dagger}=Q^{\dagger}(x, \eta) \Psi^{\dagger}, & Q^{\dagger}(x, \eta)=\sum_{n \geq 0} \eta^{-n} Q_{n}^{\dagger}(x),\end{cases}
$$

where

$$
\begin{aligned}
& P^{\dagger}(x, \eta)=\left(T^{\dagger}\right)^{-1} P(x, \eta) T^{\dagger}-\eta^{-1}\left(T^{\dagger}\right)^{-1} \frac{\partial T^{\dagger}}{\partial x_{1}} \\
& Q^{\dagger}(x, \eta)=\left(T^{\dagger}\right)^{-1} Q(x, \eta) T^{\dagger}-\eta^{-1}\left(T^{\dagger}\right)^{-1} \frac{\partial T^{\dagger}}{\partial x_{2}}
\end{aligned}
$$

In particular,

$$
P_{0}^{\dagger}=\left(\begin{array}{ccc}
0 & 1 & 0 \\
0 & 0 & 1 \\
-a_{3} & -a_{2} & 0
\end{array}\right), \quad Q_{0}^{\dagger}=\left(\begin{array}{ccc}
b_{0} & b_{1} & b_{2} \\
-b_{2} a_{3} & -b_{2} a_{2}+b_{0} & b_{1} \\
-b_{1} a_{3} & -b_{2} a_{3}-b_{1} a_{2} & -b_{2} a_{2}+b_{0}
\end{array}\right)
$$

in view of Lemmas 2.3 and 2.4. Since the system (2.1) satisfies the compatibility condition, the system (2.39) also satisfies the compatibility condition

$$
\left[P^{\dagger}, Q^{\dagger}\right]+\eta^{-1} \frac{\partial P^{\dagger}}{\partial x_{2}}-\eta^{-1} \frac{\partial Q^{\dagger}}{\partial x_{1}}=0
$$

In particular,

$$
\begin{gathered}
{\left[P_{0}^{\dagger}, Q_{0}^{\dagger}\right]=0} \\
{\left[P_{1}^{\dagger}, Q_{0}^{\dagger}\right]+\left[P_{0}^{\dagger}, Q_{1}^{\dagger}\right]+\frac{\partial P_{0}^{\dagger}}{\partial x_{2}}-\frac{\partial Q_{0}^{\dagger}}{\partial x_{1}}=0}
\end{gathered}
$$

For $k=0,1,2$, we have

$$
\begin{aligned}
\operatorname{tr}\left\{\left(P_{0}^{\dagger}\right)^{k}\right. & \left.\left(\left[P_{1}^{\dagger}, Q_{0}^{\dagger}\right]+\left[P_{0}^{\dagger}, Q_{1}^{\dagger}\right]\right)\right\} \\
& =\operatorname{tr}\left\{\left(P_{0}^{\dagger}\right)^{k} P_{1}^{\dagger} Q_{0}^{\dagger}-\left(P_{0}^{\dagger}\right)^{k} Q_{0}^{\dagger} P_{1}^{\dagger}\right\}+\operatorname{tr}\left\{\left(P_{0}^{\dagger}\right)^{k} P_{0}^{\dagger} Q_{1}^{\dagger}-\left(P_{0}^{\dagger}\right)^{k} Q_{1}^{\dagger} P_{0}^{\dagger}\right\} \\
& =\operatorname{tr}\left[\left(P_{0}^{\dagger}\right)^{k} P_{1}^{\dagger}, Q_{0}^{\dagger}\right]+\operatorname{tr}\left[P_{0}^{\dagger},\left(P_{0}^{\dagger}\right)^{k} Q_{1}^{\dagger}\right]=0
\end{aligned}
$$


in view of (2.43). Hence

$$
\operatorname{tr}\left\{\left(P_{0}^{\dagger}\right)^{k}\left(\frac{\partial P_{0}^{\dagger}}{\partial x_{2}}-\frac{\partial Q_{0}^{\dagger}}{\partial x_{1}}\right)\right\}=0
$$

for $k=0,1,2$. On the other hand, using (2.42), we obtain by straightforward computations

$$
\operatorname{tr}\left\{\left(\frac{\partial P_{0}^{\dagger}}{\partial x_{2}}-\frac{\partial Q_{0}^{\dagger}}{\partial x_{1}}\right)\right\}=\frac{\partial}{\partial x_{1}}\left(2 b_{2} a_{2}-3 b_{0}\right)
$$

$$
\operatorname{tr}\left\{P_{0}^{\dagger}\left(\frac{\partial P_{0}^{\dagger}}{\partial x_{2}}-\frac{\partial Q_{0}^{\dagger}}{\partial x_{1}}\right)\right\}=-\frac{\partial a_{2}}{\partial x_{2}}+2 b_{2} \frac{\partial a_{3}}{\partial x_{1}}+b_{1} \frac{\partial a_{2}}{\partial x_{1}}+3 \frac{\partial b_{2}}{\partial x_{1}} a_{3}+2 \frac{\partial b_{1}}{\partial x_{1}} a_{2},
$$

$$
\operatorname{tr}\left\{\left(P_{0}^{\dagger}\right)^{2}\left(\frac{\partial P_{0}^{\dagger}}{\partial x_{2}}-\frac{\partial Q_{0}^{\dagger}}{\partial x_{1}}\right)\right\}=-\frac{\partial a_{3}}{\partial x_{2}}+b_{1} \frac{\partial a_{3}}{\partial x_{1}}+3 \frac{\partial b_{1}}{\partial x_{1}} a_{3}+2 a_{2} \frac{\partial}{\partial x_{1}}\left(b_{0}-b_{2} a_{2}\right) .
$$

Using (2.45)-(2.48), we obtain (2.38).

Proposition 2.9. Assumption (ii) of Theorem 2.1 follows from assumptions (i) and (iii).

Proof. This proposition is verified by using the compatibility condition for the system (2.1). If $\left(\partial a_{3} / \partial x_{1}\right)(0)=0$, then $\left(\partial a_{3} / \partial x_{2}\right)(0) \neq 0$ in view of (iii). Using this relation and (i), we get $(2.38)$ by similar arguments to those in the proofs of Lemmas 2.3, 2.4 and 2.8. By (i) and (2.38), we find

$$
\begin{aligned}
\operatorname{det}\left(\begin{array}{ll}
\frac{\partial a_{2}}{\partial x_{1}}(0) & \frac{\partial a_{2}}{\partial x_{2}}(0) \\
\frac{\partial a_{3}}{\partial x_{1}}(0) & \frac{\partial a_{3}}{\partial x_{2}}(0)
\end{array}\right) & =\operatorname{det}\left(\begin{array}{ll}
\frac{\partial a_{2}}{\partial x_{1}}(0) & 2 b_{2}(0) \frac{\partial a_{3}}{\partial x_{1}}(0)+b_{1}(0) \frac{\partial a_{2}}{\partial x_{1}}(0) \\
\frac{\partial a_{3}}{\partial x_{1}}(0) & b_{1}(0) \frac{\partial a_{3}}{\partial x_{1}}(0)
\end{array}\right) \\
& =-2 b_{2}(0)\left(\frac{\partial a_{3}}{\partial x_{1}}(0)\right)^{2}=0
\end{aligned}
$$

This contradicts (iii). Thus $\left(\partial a_{3} / \partial x_{1}\right)(0) \neq 0$, that is, (ii) holds.

Remark 2.10. By Proposition 2.9, the assumptions of Theorem 2.1 are equivalent to

(i') $a_{2}(0)=a_{3}(0)=0$.

(ii $\left.{ }^{\prime}\right) \operatorname{det}\left(\begin{array}{ll}\frac{\partial a_{2}}{\partial x_{1}}(0) & \frac{\partial a_{2}}{\partial x_{2}}(0) \\ \frac{\partial a_{3}}{\partial x_{1}}(0) & \frac{\partial a_{3}}{\partial x_{2}}(0)\end{array}\right) \neq 0$. 
Remark 2.11. By Remark 2.7, we find that

$\left(\mathrm{i}^{\prime \prime}\right) a_{2}(0)=a_{3}(0)=0$,

(ii") $\left\{x ; 27 a_{3}(x)^{2}+4 a_{2}(x)^{3}=0\right\}$ can be transformed into $\left\{z ; 27 z_{1}^{2}+8 z_{2}^{3}=0\right\}$ by some coordinate transformation $z: \mathbb{C}_{x}^{2} \rightarrow \mathbb{C}_{z}^{2}$ near $x=0$,

are necessary conditions for $\left(\mathrm{i}^{\prime}\right)$ and ( $\left.\mathrm{ii}^{\prime}\right)$ of Remark 2.10 to hold. Furthermore, we can prove that $\left(\mathrm{i}^{\prime \prime}\right)$ and $\left(\mathrm{ii}^{\prime \prime}\right)$ are necessary and sufficient conditions for $\left(\mathrm{i}^{\prime}\right)$ and $\left(\mathrm{ii}^{\prime}\right)$.

We now see that the Stokes geometry for (2.1) can be transformed into the Stokes geometry for the Pearcey system near $x=0$ by the coordinate transformation $\widetilde{x}(x)$ given by Theorem 2.1 .

Lemma 2.12. Let $\widetilde{x}(x)$ be the coordinate transformation given by Theorem 2.1. Let $\widetilde{\xi}_{1, i}(\widetilde{x})(i=1,2,3)$ be the roots of

$$
\operatorname{det}\left(\widetilde{\xi}_{1}-\widetilde{P}_{0}(\widetilde{x})\right)=\left(\widetilde{\xi}_{1}\right)^{3}+\frac{\widetilde{x}_{2}}{2} \widetilde{\xi}_{1}+\frac{\widetilde{x}_{1}}{4}=0,
$$

and let $\widetilde{\xi}_{2, i}(\widetilde{x})$ be the roots of $\operatorname{det}\left(\widetilde{\xi}_{2}-\widetilde{Q}_{0}(\widetilde{x})\right)$, that is,

$$
\widetilde{\xi}_{2, i}(\widetilde{x})=\left(\widetilde{\xi}_{1, i}(\widetilde{x})\right)^{2}+\frac{\widetilde{x}_{2}}{3}
$$

Then

$$
\begin{aligned}
& \xi_{1, i}(x)=\widetilde{\xi}_{1, i}(\widetilde{x}(x)) \frac{\partial \widetilde{x}_{1}}{\partial x_{1}}+\widetilde{\xi}_{2, i}(\widetilde{x}(x)) \frac{\partial \widetilde{x}_{2}}{\partial x_{1}} \\
& \xi_{2, i}(x)=\widetilde{\xi}_{1, i}(\widetilde{x}(x)) \frac{\partial \widetilde{x}_{1}}{\partial x_{2}}+\widetilde{\xi}_{2, i}(\widetilde{x}(x)) \frac{\partial \widetilde{x}_{2}}{\partial x_{2}}
\end{aligned}
$$

satisfy

$$
\operatorname{det}\left(\xi_{1}-P_{0}(x)\right)=\xi_{1}^{3}+a_{2}(x) \xi_{1}+a_{3}(x)=0, \quad \xi_{2}=b_{2}(x) \xi_{1}^{2}+b_{1}(x) \xi_{1}+b_{0}(x) .
$$

Conversely, any solution of (2.52) is given by (2.51).

Using this lemma, we have

Proposition 2.13. A point $c \in D_{\rho}$ is a turning point of the system (2.1) if and only if $\widetilde{x}(c)$ is a turning point of the Pearcey system (2.13).

The proof of Lemma 2.12 and Proposition 2.13 is given in Subsection 4.1. Finally, we consider the Stokes surface for the system (2.1). 
Lemma 2.14. For $i=1,2,3$, we have

$$
\frac{\partial \xi_{2, i}}{\partial x_{1}}=\frac{\partial \xi_{1, i}}{\partial x_{2}}
$$

Proof. For simplicity, $\xi_{1, i}$ and $\xi_{2, i}$ are denoted in the proof by $\xi_{1}$ and $\xi_{2}$, respectively. We show the lemma near a point $x \in \mathbb{C}^{2}$ which satisfies $3 \xi_{1}(x)^{2}+a_{2}(x) \neq 0$, that is, outside the set of turning points for the system.

Taking the partial derivative of $\xi_{1}^{3}+a_{2}(x) \xi_{1}+a_{3}(x)=0$ with respect to the variable $x_{1}$ and $x_{2}$, we obtain

$$
\begin{aligned}
& \left(3 \xi_{1}^{2}+a_{2}\right) \frac{\partial \xi_{1}}{\partial x_{1}}+\frac{\partial a_{2}}{\partial x_{1}} \xi_{1}+\frac{\partial a_{3}}{\partial x_{1}}=0, \\
& \left(3 \xi_{1}^{2}+a_{2}\right) \frac{\partial \xi_{1}}{\partial x_{2}}+\frac{\partial a_{2}}{\partial x_{2}} \xi_{1}+\frac{\partial a_{3}}{\partial x_{2}}=0 .
\end{aligned}
$$

Since $3 \xi_{1}(x)^{2}+a_{2}(x) \neq 0$, it suffices to prove that $\left(\partial \xi_{2} / \partial x_{1}\right)(x)$ also satisfies

$$
\left(3 \xi_{1}^{2}+a_{2}\right) \frac{\partial \xi_{2}}{\partial x_{1}}+\frac{\partial a_{2}}{\partial x_{2}} \xi_{1}+\frac{\partial a_{3}}{\partial x_{2}}=0 .
$$

Using (2.35), (2.54) and Lemma 2.8, we obtain

$$
\begin{aligned}
& \left(3 \xi_{1}^{2}+a_{2}\right) \frac{\partial \xi_{2}}{\partial x_{1}}+\frac{\partial a_{2}}{\partial x_{2}} \xi_{1}+\frac{\partial a_{3}}{\partial x_{2}} \\
= & \left(3 \xi_{1}^{2}+a_{2}\right)\left\{\left(2 b_{2} \xi_{1}+b_{1}\right) \frac{\partial \xi_{1}}{\partial x_{1}}+\frac{\partial b_{2}}{\partial x_{1}} \xi_{1}^{2}+\frac{\partial b_{1}}{\partial x_{1}} \xi_{1}+\frac{\partial b_{0}}{\partial x_{1}}\right\}+\frac{\partial a_{2}}{\partial x_{2}} \xi_{1}+\frac{\partial a_{3}}{\partial x_{2}} \\
= & \left(2 b_{2} \xi_{1}+b_{1}\right)\left(-\frac{\partial a_{2}}{\partial x_{1}} \xi_{1}-\frac{\partial a_{3}}{\partial x_{1}}\right)+\left(3 \xi_{1}^{2}+a_{2}\right)\left(\frac{\partial b_{2}}{\partial x_{1}} \xi_{1}^{2}+\frac{\partial b_{1}}{\partial x_{1}} \xi_{1}+\frac{\partial b_{0}}{\partial x_{1}}\right)+\frac{\partial a_{2}}{\partial x_{2}} \xi_{1}+\frac{\partial a_{3}}{\partial x_{2}} \\
= & \left(-2 b_{2} \frac{\partial a_{2}}{\partial x_{1}}-2 \frac{\partial b_{2}}{\partial x_{1}} a_{2}+3 \frac{\partial b_{0}}{\partial x_{1}}\right) \xi_{1}^{2} \\
& +\left(-2 b_{2} \frac{\partial a_{3}}{\partial x_{1}}-b_{1} \frac{\partial a_{2}}{\partial x_{1}}-3 \frac{\partial b_{2}}{\partial x_{1}} a_{3}-2 \frac{\partial b_{1}}{\partial x_{1}} a_{2}+\frac{\partial a_{2}}{\partial x_{2}}\right) \xi_{1} \\
& +\left(-b_{1} \frac{\partial a_{3}}{\partial x_{1}}-3 \frac{\partial b_{1}}{\partial x_{1}} a_{3}+\frac{\partial b_{0}}{\partial x_{1}} a_{2}+\frac{\partial a_{3}}{\partial x_{2}}\right)=0
\end{aligned}
$$

Note that Lemma 2.14 guarantees that $\xi_{1, i} d x_{1}+\xi_{2, i} d x_{2}$ is a closed 1-form. Based on this fact, the Stokes surface for (2.1) is defined as follows:

Definition 2.15. Let $c \in \mathbb{C}^{2}$ be a turning point which satisfies (2.36). A Stokes surface for the system (2.1) emanating from $x=c$ is a real 3-dimensional surface defined by

$$
\Im \int_{c}^{x}\left\{\left(\xi_{1, i} d x_{1}+\xi_{2, i} d x_{2}\right)-\left(\xi_{1, i^{\prime}} d x_{1}+\xi_{2, i^{\prime}} d x_{2}\right)\right\}=0
$$


By using the coordinate transformation $\widetilde{x}(x)$ of Theorem 2.1, we have

Proposition 2.16. Let $x_{0}$ be a point in $D_{\rho}$. Then

$$
\int_{x_{0}}^{x}\left(\xi_{1, i} d x_{1}+\xi_{2, i} d x_{2}\right)=\int_{\widetilde{x}\left(x_{0}\right)}^{\widetilde{x}(x)}\left(\widetilde{\xi}_{1, i} d \widetilde{x}_{1}+\widetilde{\xi}_{2, i} d \widetilde{x}_{2}\right) .
$$

In particular, a point $x \in D_{\rho}$ is in the Stokes surface for the system (2.1) if and only if $\widetilde{x}(x)$ is in the Stokes surface for the Pearcey system (2.13).

Proposition 2.16 is an immediate consequence of Lemma 2.12 and Proposition 2.13 .

\section{§2.2. Derivation of Theorem 2.2 from Theorem 2.1}

In this subsection, we give the proof of Theorem 2.2 .

By assumption $\left(i^{\star}\right)$ and using the arguments of [W1, Chapter VII], [W2, Chapter II] and [T], we can verify that there exist a sufficiently small positive constant $0<\rho<\rho_{0}$ and an infinite series of $m \times m$ matrices $\left\{T_{n}^{\star}(x)\right\}_{n \geq 0}$ satisfying:

- Every entry of $T_{n}^{\star}(x)$ is holomorphic in $D_{\rho}$ and $\operatorname{det} T_{0}^{\star}(x) \neq 0\left(x \in D_{\rho}\right)$.

- $T_{n}^{\star}(x)(n \geq 0)$ satisfy

$$
\left\|T_{n}^{\star}\right\|_{\rho, \rho} \leq C^{\star}\left(\alpha^{\star}\right)^{n} n !
$$

with some positive constants $C^{\star}$ and $\alpha^{\star}$.

- The formal transformation

$$
\Psi=T^{\star}(x, \eta) \Psi^{\star}, \quad T^{\star}(x, \eta)=\sum_{n \geq 0} \eta^{-n} T_{n}^{\star}(x),
$$

transforms the first equation of (2.18) into

$$
\eta^{-1} \frac{\partial}{\partial x_{1}} \Psi^{\star}=P^{\star}(x, \eta) \Psi^{\star}, \quad P^{\star}(x, \eta)=\sum_{n \geq 0} \eta^{-n} P_{n}^{\star}(x),
$$

where $P_{n}^{\star}(x)$ is a block-diagonal matrix,

$$
P_{n}^{\star}(x)=\left(\begin{array}{ll}
P_{n}^{\star(1)}(x) & \\
& P_{n}^{\star(2)}(x)
\end{array}\right),
$$

$P_{n}^{\star(1)}(x)$ is a $3 \times 3$ matrix, $P_{n}^{\star(2)}(x)$ is an $(m-3) \times(m-3)$ matrix, and all coefficients $P_{n}^{\star(1)}(x)$ and $P_{n}^{\star(2)}(x)$ are holomorphic in $D_{\rho}$ and satisfy

$$
\left\|P_{n}^{\star(j)}\right\|_{\rho, \rho} \leq \widetilde{C}^{\star}\left(\widetilde{\alpha}^{\star}\right)^{n} n ! \quad(j=1,2)
$$

with some positive constants $\widetilde{C}^{\star}$ and $\widetilde{\alpha}^{\star}$. 
- $D^{(1)}\left(x, \xi_{1}\right)=\operatorname{det}\left(\xi_{1}-P_{0}^{\star(1)}(x)\right)$ and $D^{(2)}\left(x, \xi_{1}\right)=\operatorname{det}\left(\xi_{1}-P_{0}^{\star(2)}(x)\right)$ satisfy

$$
\begin{aligned}
D\left(x, \xi_{1}\right) & =D^{(1)}\left(x, \xi_{1}\right) D^{(2)}\left(x, \xi_{1}\right), \\
D^{(1)}\left(0, \xi_{1}^{\star}\right)=\frac{\partial D^{(1)}}{\partial \xi_{1}}\left(0, \xi_{1}^{\star}\right) & =\frac{\partial^{2} D^{(1)}}{\partial \xi_{1}^{2}}\left(0, \xi_{1}^{\star}\right)=0, \quad \frac{\partial^{3} D^{(1)}}{\partial \xi_{1}^{3}}\left(0, \xi_{1}^{\star}\right) \neq 0, \\
D^{(2)}\left(0, \xi_{1}^{\star}\right) \neq 0 . &
\end{aligned}
$$

(The proof of the estimates (2.58), (2.62) is provided in [T].)

Furthermore, by using the argument of [T], the second equation of (2.18) can be transformed by (2.59) into

$$
\eta^{-1} \frac{\partial}{\partial x_{2}} \Psi^{\star}=Q^{\star}(x, \eta) \Psi^{\star}, \quad Q^{\star}(x, \eta)=\sum_{n \geq 0} \eta^{-n} Q_{n}^{\star}(x),
$$

where $Q_{n}^{\star}(x)$ is a block-diagonal matrix,

$$
Q_{n}^{\star}(x)=\left(\begin{array}{ll}
Q_{n}^{\star(1)}(x) & \\
& Q_{n}^{\star(2)}(x)
\end{array}\right),
$$

$Q_{n}^{\star(1)}(x)$ is a $3 \times 3$ matrix, $Q_{n}^{\star(2)}(x)$ is an $(m-3) \times(m-3)$ matrix, and (after changing $\rho, \widetilde{C}^{\star}$ and $\widetilde{\alpha}^{\star}$ if necessary) all coefficients $Q_{n}^{\star(1)}(x)$ and $Q_{n}^{\star(2)}(x)$ are holomorphic in $D_{\rho}$ and satisfy

$$
\left\|Q_{n}^{\star(j)}\right\|_{\rho, \rho} \leq \widetilde{C}^{\star}\left(\widetilde{\alpha}^{\star}\right)^{n} n ! \quad(j=1,2) .
$$

Thus what remains to be proved is the last claim of Theorem 2.2 for the completely integrable system

$$
\begin{cases}\eta^{-1} \frac{\partial}{\partial x_{1}} \Psi^{\star(1)}=P^{\star(1)}(x, \eta) \Psi^{\star(1)}, & P^{\star(1)}(x, \eta)=\sum_{n \geq 0} \eta^{-n} P_{n}^{\star(1)}(x), \\ \eta^{-1} \frac{\partial}{\partial x_{2}} \Psi^{\star(1)}=Q^{\star(1)}(x, \eta) \Psi^{\star(1)}, & Q^{\star(1)}(x, \eta)=\sum_{n \geq 0} \eta^{-n} Q_{n}^{\star(1)}(x) .\end{cases}
$$

Let $a_{k}^{\star}(x)(k=1,2,3)$ be holomorphic functions near $x=0$ defined by

$$
\begin{aligned}
D^{(1)}\left(x, \xi_{1}\right) & =\operatorname{det}\left(\xi_{1}-P_{0}^{\star(1)}(x)\right) \\
& =\left(\xi_{1}-\xi_{1}^{\star}\right)^{3}+a_{1}^{\star}(x)\left(\xi_{1}-\xi_{1}^{\star}\right)^{2}+a_{2}^{\star}(x)\left(\xi_{1}-\xi_{1}^{\star}\right)+a_{3}^{\star}(x) .
\end{aligned}
$$

Then, by $(2.64), a_{k}^{\star}(x)(k=1,2,3)$ satisfy

$$
a_{k}^{\star}(0)=0 .
$$


Using (2.63), (2.64) and (2.70), we have

$$
\begin{aligned}
\frac{\partial D}{\partial x_{1}}\left(0, \xi_{1}^{\star}\right) & =\frac{\partial D^{(1)}}{\partial x_{1}}\left(0, \xi_{1}^{\star}\right) D^{(2)}\left(0, \xi_{1}^{\star}\right)+D^{(1)}\left(0, \xi_{1}^{\star}\right) \frac{\partial D^{(2)}}{\partial x_{1}}\left(0, \xi_{1}^{\star}\right) \\
& =\frac{\partial a_{3}^{\star}}{\partial x_{1}}(0) D^{(2)}\left(0, \xi_{1}^{\star}\right) .
\end{aligned}
$$

Hence, by assumption ( $\left.\mathrm{ii}^{\star}\right)$ and (2.65), we find

$$
\frac{\partial a_{3}^{\star}}{\partial x_{1}}(0) \neq 0 .
$$

On the other hand, using (2.63), (2.64), (2.70) and

$$
\frac{\partial^{2} D^{(1)}}{\partial x_{j} \partial \xi_{1}}\left(0, \xi_{1}^{\star}\right)=\frac{\partial a_{2}^{\star}}{\partial x_{j}}(0),
$$

we have

$$
\left.\operatorname{det}\left(\begin{array}{cc}
\frac{\partial D}{\partial x_{1}} & \frac{\partial D}{\partial x_{2}} \\
\frac{\partial^{2} D}{\partial x_{1} \partial \xi_{1}} & \frac{\partial^{2} D}{\partial x_{2} \partial \xi_{1}}
\end{array}\right)\right|_{\left(x, \xi_{1}\right)=\left(0, \xi_{1}^{\star}\right)}=D^{(2)}\left(0, \xi_{1}^{\star}\right)^{2} \operatorname{det}\left(\begin{array}{ll}
\frac{\partial a_{3}^{\star}}{\partial x_{1}}(0) & \frac{\partial a_{3}^{\star}}{\partial x_{2}}(0) \\
\frac{\partial a_{2}^{\star}}{\partial x_{1}}(0) & \frac{\partial a_{2}^{\star}}{\partial x_{2}}(0)
\end{array}\right) .
$$

Hence, by assumption (iii $\left.{ }^{\star}\right)$ and (2.65),

$$
\operatorname{det}\left(\begin{array}{ll}
\frac{\partial a_{3}^{\star}}{\partial x_{1}}(0) & \frac{\partial a_{3}^{\star}}{\partial x_{2}}(0) \\
\frac{\partial a_{2}^{\star}}{\partial x_{1}}(0) & \frac{\partial a_{2}^{\star}}{\partial x_{2}}(0)
\end{array}\right) \neq 0
$$

The gauge transformation $\Psi^{\star(1)}=\exp \left\{\eta \int^{x} \operatorname{tr}\left(P_{0}^{\star(1)}(x) d x_{1}+Q_{0}^{\star(1)}(x) d x_{2}\right) / 3\right\} \Phi^{\star(1)}$ transforms (2.69) into

$$
\left\{\begin{array}{l}
\eta^{-1} \frac{\partial}{\partial x_{1}} \Phi^{\star(1)}=\left(P^{\star(1)}(x, \eta)-\frac{\operatorname{tr} P_{0}^{\star(1)}(x)}{3}\right) \Phi^{\star(1)}, \\
\eta^{-1} \frac{\partial}{\partial x_{2}} \Phi^{\star(1)}=\left(Q^{\star(1)}(x, \eta)-\frac{\operatorname{tr} Q_{0}^{\star(1)}(x)}{3}\right) \Phi^{\star(1)} .
\end{array}\right.
$$

Let $\widetilde{a}_{2}^{\star}(x)$ and $\widetilde{a}_{3}^{\star}(x)$ be holomorphic functions defined by

$$
\operatorname{det}\left\{\xi_{1}-\left(P_{0}^{\star(1)}(x)-\frac{\operatorname{tr} P_{0}^{\star(1)}(x)}{3}\right)\right\}=\xi_{1}^{3}+\widetilde{a}_{2}^{\star}(x) \xi_{1}+\widetilde{a}_{3}^{\star}(x) .
$$

Then

$$
\widetilde{a}_{2}^{\star}(x)=a_{2}^{\star}(x)-\frac{a_{1}^{\star}(x)^{2}}{3}, \quad \widetilde{a}_{3}^{\star}(x)=a_{3}^{\star}(x)-\frac{a_{1}^{\star}(x) a_{2}^{\star}(x)}{3}+\frac{2 a_{1}^{\star}(x)^{3}}{27} .
$$


Hence, by (2.71), (2.73) and (2.74),

$$
\widetilde{a}_{2}^{\star}(0)=\widetilde{a}_{3}^{\star}(0)=0, \quad \frac{\partial \widetilde{a}_{3}^{\star}}{\partial x_{1}}(0) \neq 0, \quad \operatorname{det}\left(\begin{array}{ll}
\frac{\partial \widetilde{a}_{2}^{\star}}{\partial x_{1}}(0) & \frac{\partial \widetilde{a}_{2}^{\star}}{\partial x_{2}}(0) \\
\frac{\partial \widetilde{a}_{3}^{\star}}{\partial x_{1}}(0) & \frac{\partial \widetilde{a}_{3}^{\star}}{\partial x_{2}}(0)
\end{array}\right) \neq 0 .
$$

That is, (2.75) satisfies assumptions (i)-(iii) of Theorem 2.1. Thus the system (2.69) can be transformed into the Pearcey system near $x=0$ thanks to Theorem 2.1. This completes the proof of Theorem 2.2.

\section{§3. Examples}

\section{$\S 3.1$. The $(1,4)$ hypergeometric system}

Let us consider the system

$$
\begin{gathered}
\left\{\begin{array}{l}
\eta^{-1} \frac{\partial}{\partial x_{1}} \Psi=P_{0}(x) \Psi \\
\eta^{-1} \frac{\partial}{\partial x_{2}} \Psi=\left(Q_{0}(x)+\eta^{-1} Q_{1}(x)\right) \Psi
\end{array}\right. \\
P_{0}=\left(\begin{array}{ccc}
2 x_{2} / 9 & 1 & 0 \\
0 & 2 x_{2} / 9 & 1 \\
\alpha / 3 & -x_{1} / 3 & -4 x_{2} / 9
\end{array}\right), \\
Q_{0}=P_{0}^{2}-\frac{4}{9} x_{2} P_{0}+\frac{2}{9} x_{1}-\frac{8}{81} x_{2}^{2}, \quad Q_{1}=\frac{\partial P_{0}}{\partial x_{1}}
\end{gathered}
$$

where $\alpha$ is a complex constant. This system is equivalent to the following holonomic system:

$$
\left\{\begin{array}{l}
\left(\frac{\partial^{3}}{\partial x_{1}^{3}}+\frac{2}{3} x_{2} \eta \frac{\partial^{2}}{\partial x_{1}^{2}}+\frac{1}{3} x_{1} \eta^{2} \frac{\partial}{\partial x_{1}}-\frac{\alpha}{3} \eta^{3}\right) \psi=0 \\
\left(\eta \frac{\partial}{\partial x_{2}}-\frac{\partial^{2}}{\partial x_{1}^{2}}\right) \psi=0
\end{array}\right.
$$

which belongs to the class of hypergeometric systems of two variables studied in $[\mathrm{OK}]$. Note that (3.3) has the following solution:

$$
\psi=\int \exp \left\{\eta\left(t^{3}+x_{2} t^{2}+x_{1} t\right)\right\} t^{-\eta \alpha-1} d t
$$

Since the system (3.3) and its solution (3.4) are determined by the partition " $(1,4)$ " of the natural number 5 , we call $(3.1)$ the $(1,4)$ hypergeometric system. In what follows, we assume $\alpha \neq 0$. 
We first study the turning points for the $(1,4)$ hypergeometric system. Let $\xi_{1}$ (resp., $\xi_{2}$ ) be an eigenvalue of $P_{0}$ (resp., $Q_{0}$ ). Then

$$
\begin{aligned}
& \operatorname{det}\left(\xi_{1}-P_{0}(x)\right)=\xi_{1}^{3}+a_{2}(x) \xi_{1}+a_{3}(x)=0, \\
& a_{2}(x)=\frac{1}{3} x_{1}-\frac{4}{27} x_{2}^{2}, \quad a_{3}(x)=\frac{16}{729} x_{2}^{3}-\frac{2}{27} x_{1} x_{2}-\frac{\alpha}{3}, \\
& \xi_{2}=\xi_{1}^{2}-\frac{4}{9} x_{2} \xi_{1}+\frac{2}{9} x_{1}-\frac{8}{81} x_{2}^{2} .
\end{aligned}
$$

In view of $(3.7)$ we find that a turning point for the $(1,4)$ hypergeometric system is given by a zero of the discriminant $27 a_{3}(x)^{2}+4 a_{2}(x)^{3}$ of (3.5). A singular point of the set of turning points for the $(1,4)$ hypergeometric system is given by

$$
a_{2}(x)=a_{3}(x)=0,
$$

that is,

$$
x=\left(3^{4 / 3} \alpha^{2 / 3} e^{4 \pi \sqrt{-1}(j-1) / 3},-\frac{3^{5 / 3}}{2} \alpha^{1 / 3} e^{2 \pi \sqrt{-1}(j-1) / 3}\right)=: c_{j} \quad(j=1,2,3) .
$$

Furthermore,

$$
\begin{aligned}
& \frac{\partial a_{3}}{\partial x_{1}}\left(c_{j}\right)=3^{-4 / 3} \alpha^{2 / 3} e^{4 \pi \sqrt{-1}(j-1) / 3} \neq 0, \\
& \operatorname{det}\left(\begin{array}{ll}
\frac{\partial a_{2}}{\partial x_{1}}\left(c_{j}\right) & \frac{\partial a_{2}}{\partial x_{2}}\left(c_{j}\right) \\
\frac{\partial a_{3}}{\partial x_{1}}\left(c_{j}\right) & \frac{\partial a_{3}}{\partial x_{2}}\left(c_{j}\right)
\end{array}\right)=-\frac{2}{81}\left(3^{4 / 3} \alpha^{2 / 3} e^{4 \pi \sqrt{-1}(j-1) / 3}\right) \neq 0 .
\end{aligned}
$$

Hence in a sufficiently small neighborhood of $x=c_{j}(j=1,2,3)$ the $(1,4)$ hypergeometric system can be transformed into the Pearcey system.

\section{$\S 3.2$. The $(2,3)$ hypergeometric system}

Next we consider the system

$$
\begin{gathered}
\left\{\begin{array}{l}
\eta^{-1} \frac{\partial}{\partial x_{1}} \Psi=\left(P_{0}(x)+\eta^{-1} P_{1}(x)\right) \Psi, \\
\eta^{-1} \frac{\partial}{\partial x_{2}} \Psi=Q_{0}(x) \Psi,
\end{array}\right. \\
P_{0}=\left(\begin{array}{ccc}
x_{1} / 6 & 1 & 0 \\
0 & x_{1} / 6 & 1 \\
x_{2} / 2 & \alpha / 2 & -x_{1} / 3
\end{array}\right), \quad P_{1}=\left(\begin{array}{ccc}
0 & 0 & 0 \\
0 & 0 & 0 \\
0 & -1 / 2 & 0
\end{array}\right), \\
Q_{0}=\frac{2}{x_{2}} P_{0}^{2}+\frac{x_{1}}{3 x_{2}} P_{0}-\frac{x_{1}^{2}}{9 x_{2}}-\frac{2 \alpha}{3 x_{2}},
\end{gathered}
$$

where $\alpha$ is a complex constant. This system is equivalent to the holonomic system 


$$
\left\{\begin{array}{l}
\left(\frac{\partial^{3}}{\partial x_{1}^{3}}+\frac{x_{1}}{2} \eta \frac{\partial^{2}}{\partial x_{1}^{2}}+\frac{-\alpha+\eta^{-1}}{2} \eta^{2} \frac{\partial}{\partial x_{1}}-\frac{x_{2}}{2} \eta^{3}\right) \psi=0, \\
\left(\frac{\partial^{2}}{\partial x_{1} \partial x_{2}}-\eta^{2}\right) \psi=0,
\end{array}\right.
$$

which also belongs to the class of hypergeometric systems in two variables studied in $[\mathrm{OK}]$. Since (3.10) has the solution

$$
\psi=\int \exp \left\{\eta\left(t^{2}+x_{1} t+x_{2} / t\right)\right\} t^{-\eta \alpha-1} d t
$$

and it is determined by the partition " $(2,3)$ " of the natural number 5 , we call (3.8) the $(2,3)$ hypergeometric system. In what follows, we assume $x_{2} \neq 0$ and $\alpha \neq 0$.

Similarly to the preceding subsection we study the turning points for the $(2,3)$ hypergeometric system. Let $\xi_{1}$ (resp., $\xi_{2}$ ) be an eigenvalue of $P_{0}$ (resp., $Q_{0}$ ). Then

$$
\begin{aligned}
& \operatorname{det}\left(\xi_{1}-P_{0}(x)\right)=\xi_{1}^{3}+a_{2}(x) \xi_{1}+a_{3}(x), \\
& a_{2}(x)=-\frac{x_{1}^{2}}{12}-\frac{\alpha}{2}, \quad a_{3}(x)=\frac{x_{1}^{3}}{108}+\frac{\alpha x_{1}}{12}-\frac{x_{2}}{2}, \\
& \xi_{2}=\frac{2}{x_{2}} \xi_{1}^{2}+\frac{x_{1}}{3 x_{2}} \xi_{1}-\frac{x_{1}^{2}}{9 x_{2}}-\frac{2 \alpha}{3 x_{2}} .
\end{aligned}
$$

Thanks to (3.14) a turning point for the $(2,3)$ hypergeometric system is given by a zero of the discriminant $27 a_{3}(x)^{2}+4 a_{2}(x)^{3}$ of (3.12). A singular point of the set of turning points for the $(2,3)$ hypergeometric system is given by

$$
a_{2}(x)=a_{3}(x)=0,
$$

that is,

$$
x= \pm\left(\sqrt{-6 \alpha}, \frac{\alpha}{18} \sqrt{-6 \alpha}\right)=: c_{ \pm} .
$$

Since

$$
\frac{\partial a_{3}}{\partial x_{1}}\left(c_{ \pm}\right)=-\frac{\alpha}{12} \neq 0, \quad \operatorname{det}\left(\begin{array}{ll}
\frac{\partial a_{2}}{\partial x_{1}}\left(c_{ \pm}\right) & \frac{\partial a_{2}}{\partial x_{2}}\left(c_{ \pm}\right) \\
\frac{\partial a_{3}}{\partial x_{1}}\left(c_{ \pm}\right) & \frac{\partial a_{3}}{\partial x_{2}}\left(c_{ \pm}\right)
\end{array}\right)= \pm \frac{\sqrt{-6 \alpha}}{12} \neq 0
$$

in a sufficiently small neighborhood of $x=c_{ \pm}$the $(2,3)$ hypergeometric system can be transformed into the Pearcey system.

\section{$\S 4$. Construction of the transformation}

In this section, we discuss the construction of $\widetilde{x}(x)$ and $\left\{T_{n}(x)\right\}_{n \geq 0}$. The estimate (2.11) for $T_{n}(x)$ will be verified in Section 5 . 
Suppose that the system (2.13) is obtained from (2.1) through the transformation

$$
\Psi(x, \eta)=T(x, \eta) \widetilde{\Psi}(\widetilde{x}(x), \eta), \quad \widetilde{x}(x)=\left(\widetilde{x}_{1}(x), \widetilde{x}_{2}(x)\right), \quad T(x, \eta)=\sum_{n \geq 0} \eta^{-n} T_{n}(x) .
$$

Then $\widetilde{x}_{i}(x)(i=1,2)$ and $T(x, \eta)$ should satisfy

$$
\left\{\begin{array}{l}
T\left(\frac{\partial \widetilde{x}_{1}}{\partial x_{1}} \widetilde{P}+\frac{\partial \widetilde{x}_{2}}{\partial x_{1}} \widetilde{Q}\right)+\eta^{-1} \frac{\partial T}{\partial x_{1}}=P T \\
T\left(\frac{\partial \widetilde{x}_{1}}{\partial x_{2}} \widetilde{P}+\frac{\partial \widetilde{x}_{2}}{\partial x_{2}} \widetilde{Q}\right)+\eta^{-1} \frac{\partial T}{\partial x_{2}}=Q T
\end{array}\right.
$$

that is,

$$
\begin{aligned}
& \left\{\begin{array}{l}
T_{0}\left(\frac{\partial \widetilde{x}_{1}}{\partial x_{1}} \widetilde{P}_{0}+\frac{\partial \widetilde{x}_{2}}{\partial x_{1}} \widetilde{Q}_{0}\right)=P_{0} T_{0}, \\
T_{0}\left(\frac{\partial \widetilde{x}_{1}}{\partial x_{2}} \widetilde{P}_{0}+\frac{\partial \widetilde{x}_{2}}{\partial x_{2}} \widetilde{Q}_{0}\right)=Q_{0} T_{0},
\end{array}\right. \\
& \left\{\begin{array}{l}
\sum_{j=0}^{n} T_{j}\left(\frac{\partial \widetilde{x}_{1}}{\partial x_{1}} \widetilde{P}_{n-j}+\frac{\partial \widetilde{x}_{2}}{\partial x_{1}} \widetilde{Q}_{n-j}\right)+\frac{\partial T_{n-1}}{\partial x_{1}}=\sum_{j=0}^{n} P_{n-j} T_{j}, \\
\sum_{j=0}^{n} T_{j}\left(\frac{\partial \widetilde{x}_{1}}{\partial x_{2}} \widetilde{P}_{n-j}+\frac{\partial \widetilde{x}_{2}}{\partial x_{2}} \widetilde{Q}_{n-j}\right)+\frac{\partial T_{n-1}}{\partial x_{2}}=\sum_{j=0}^{n} Q_{n-j} T_{j}, \quad(n \geq 1) .
\end{array}\right.
\end{aligned}
$$

\section{$\S 4.1$. Construction of the transformation, I}

In this subsection, we prove

Proposition 4.1. Let $a_{2}(x)$ and $a_{3}(x)$ be holomorphic functions defined by $\operatorname{det}\left(\xi_{1}-P_{0}(x)\right)=\xi_{1}^{3}+a_{2}(x) \xi_{1}+a_{3}(x)$. Suppose that

(i) $a_{2}(0)=a_{3}(0)=0$.

(ii) $\frac{\partial a_{3}}{\partial x_{1}}(0) \neq 0$.

(iii) $\operatorname{det}\left(\begin{array}{ll}\frac{\partial a_{2}}{\partial x_{1}}(0) & \frac{\partial a_{2}}{\partial x_{2}}(0) \\ \frac{\partial a_{3}}{\partial x_{1}}(0) & \frac{\partial a_{3}}{\partial x_{2}}(0)\end{array}\right) \neq 0$.

Then there exist a sufficiently small positive constant $0<\rho<\rho_{0}$, holomorphic functions $\widetilde{x}_{i}(x)(i=1,2)$ in $D_{\rho}$, and a $3 \times 3$ matrix $T_{0}(x)$ such that:

- $\widetilde{x}_{1}(0)=\widetilde{x}_{2}(0)=0$.

- $\widetilde{x}(x)=\left(\widetilde{x}_{1}(x), \widetilde{x}_{2}(x)\right)$ is a biholomorphic map from $D_{\rho}$ to $\widetilde{x}\left(D_{\rho}\right)$. 
- Every entry of $T_{0}(x)$ is holomorphic in $D_{\rho}$ and $\operatorname{det} T_{0}(x) \neq 0\left(x \in D_{\rho}\right)$.

- The transformation

$$
\Psi(x, \eta)=T_{0}(x) \widetilde{\Psi}(\widetilde{x}(x), \eta)
$$

transforms (2.1) into

$$
\begin{cases}\eta^{-1} \frac{\partial}{\partial \widetilde{x}_{1}} \widetilde{\Psi}=\widetilde{P}(\widetilde{x}, \eta) \widetilde{\Psi}, & \widetilde{P}(\widetilde{x}, \eta)=\sum_{n \geq 0} \eta^{-n} \widetilde{P}_{n}(\widetilde{x}) \\ \eta^{-1} \frac{\partial}{\partial \widetilde{x}_{2}} \widetilde{\Psi}=\widetilde{Q}(\widetilde{x}, \eta) \widetilde{\Psi}, & \widetilde{Q}(\widetilde{x}, \eta)=\sum_{n \geq 0} \eta^{-n} \widetilde{Q}_{n}(\widetilde{x})\end{cases}
$$

where

$$
\widetilde{P}_{0}=\left(\begin{array}{ccc}
0 & 1 & 0 \\
0 & 0 & 1 \\
-\widetilde{x}_{1} / 4 & -\widetilde{x}_{2} / 2 & 0
\end{array}\right), \quad \widetilde{Q}_{0}=\widetilde{P}_{0}^{2}+\frac{\widetilde{x}_{2}}{3} .
$$

Here $\widetilde{P}_{n}(x)$ and $\widetilde{Q}_{n}(x)$ are $3 \times 3$ matrices with holomorphic entries in $D_{\rho}$, and

$$
\left\|\widetilde{P}_{n}\right\|_{\rho, \rho},\left\|\widetilde{Q}_{n}\right\|_{\rho, \rho} \leq \widetilde{C} \widetilde{\alpha}^{n} n ! \quad(n \geq 0)
$$

with some positive constants $\widetilde{C}$ and $\widetilde{\alpha}$.

Since the system (4.2) is obtained from (2.1) through the transformation (4.1), $\widetilde{x}_{i}(x)(i=1,2)$ and $T_{0}(x)$ should satisfy

$$
\left\{\begin{array}{l}
T_{0}\left(\frac{\partial \widetilde{x}_{1}}{\partial x_{1}} \widetilde{P}+\frac{\partial \widetilde{x}_{2}}{\partial x_{1}} \widetilde{Q}\right)+\eta^{-1} \frac{\partial T_{0}}{\partial x_{1}}=P T_{0} \\
T_{0}\left(\frac{\partial \widetilde{x}_{1}}{\partial x_{2}} \widetilde{P}+\frac{\partial \widetilde{x}_{2}}{\partial x_{2}} \widetilde{Q}\right)+\eta^{-1} \frac{\partial T_{0}}{\partial x_{2}}=Q T_{0}
\end{array}\right.
$$

that is,

$$
\begin{aligned}
& \left\{\begin{array}{l}
T_{0}\left(\frac{\partial \widetilde{x}_{1}}{\partial x_{1}} \widetilde{P}_{0}+\frac{\partial \widetilde{x}_{2}}{\partial x_{1}} \widetilde{Q}_{0}\right)=P_{0} T_{0}, \\
T_{0}\left(\frac{\partial \widetilde{x}_{1}}{\partial x_{2}} \widetilde{P}_{0}+\frac{\partial \widetilde{x}_{2}}{\partial x_{2}} \widetilde{Q}_{0}\right)=Q_{0} T_{0},
\end{array}\right. \\
& \left\{\begin{array}{l}
T_{0}\left(\frac{\partial \widetilde{x}_{1}}{\partial x_{1}} \widetilde{P}_{1}+\frac{\partial \widetilde{x}_{2}}{\partial x_{1}} \widetilde{Q}_{1}\right)+\frac{\partial T_{0}}{\partial x_{1}}=P_{1} T_{0}, \\
T_{0}\left(\frac{\partial \widetilde{x}_{1}}{\partial x_{2}} \widetilde{P}_{1}+\frac{\partial \widetilde{x}_{2}}{\partial x_{2}} \widetilde{Q}_{1}\right)+\frac{\partial T_{0}}{\partial x_{2}}=Q_{1} T_{0},
\end{array}\right. \\
& \left\{\begin{array}{l}
T_{0}\left(\frac{\partial \widetilde{x}_{1}}{\partial x_{1}} \widetilde{P}_{n}+\frac{\partial \widetilde{x}_{2}}{\partial x_{1}} \widetilde{Q}_{n}\right)=P_{n} T_{0}, \\
T_{0}\left(\frac{\partial \widetilde{x}_{1}}{\partial x_{2}} \widetilde{P}_{n}+\frac{\partial \widetilde{x}_{2}}{\partial x_{2}} \widetilde{Q}_{n}\right)=Q_{n} T_{0}, \quad(n \geq 2) .
\end{array}\right.
\end{aligned}
$$


We first show

Lemma 4.2. $\widetilde{x}_{i}(x)(i=1,2)$ satisfy the following system of nonlinear partial differential equations:

$$
\left\{\begin{array}{l}
\frac{\widetilde{x}_{2}^{2}}{12}\left(\frac{\partial \widetilde{x}_{2}}{\partial x_{1}}\right)^{2}-\frac{3}{4} \widetilde{x}_{1} \frac{\partial \widetilde{x}_{1}}{\partial x_{1}} \frac{\partial \widetilde{x}_{2}}{\partial x_{1}}-\frac{\widetilde{x}_{2}}{2}\left(\frac{\partial \widetilde{x}_{1}}{\partial x_{1}}\right)^{2}+a_{2}(x)=0 \\
\frac{\widetilde{x}_{1}^{2}}{16}\left(\frac{\partial \widetilde{x}_{2}}{\partial x_{1}}\right)^{3}+\frac{\widetilde{x}_{2}^{3}}{108}\left(\frac{\partial \widetilde{x}_{2}}{\partial x_{1}}\right)^{3}-\frac{\widetilde{x}_{1}}{4}\left(\frac{\partial \widetilde{x}_{1}}{\partial x_{1}}\right)^{3} \\
\quad+\frac{\widetilde{x}_{1} \widetilde{x}_{2}}{8} \frac{\partial \widetilde{x}_{1}}{\partial x_{1}}\left(\frac{\partial \widetilde{x}_{2}}{\partial x_{1}}\right)^{2}+\frac{\widetilde{x}_{2}^{2}}{6}\left(\frac{\partial \widetilde{x}_{1}}{\partial x_{1}}\right)^{2} \frac{\partial \widetilde{x}_{2}}{\partial x_{1}}+a_{3}(x)=0 \\
\frac{\partial \widetilde{x}_{1}}{\partial x_{2}}=b_{2}(x)\left\{-\frac{\widetilde{x}_{2}}{3} \frac{\partial \widetilde{x}_{1}}{\partial x_{1}} \frac{\partial \widetilde{x}_{2}}{\partial x_{1}}-\frac{\widetilde{x}_{1}}{4}\left(\frac{\partial \widetilde{x}_{2}}{\partial x_{1}}\right)^{2}\right\}+b_{1}(x) \frac{\partial \widetilde{x}_{1}}{\partial x_{1}} \\
\frac{\partial \widetilde{x}_{2}}{\partial x_{2}}=b_{2}(x)\left\{\left(\frac{\partial \widetilde{x}_{1}}{\partial x_{1}}\right)^{2}+\frac{\widetilde{x}_{2}}{6}\left(\frac{\partial \widetilde{x}_{2}}{\partial x_{1}}\right)^{2}\right\}+b_{1}(x) \frac{\partial \widetilde{x}_{2}}{\partial x_{1}} .
\end{array}\right.
$$

Proof. It follows from (4.3) and the first equation of (4.5) that

$$
P_{0} T_{0}=T_{0}\left(\frac{\partial \widetilde{x}_{2}}{\partial x_{1}} \widetilde{P}_{0}^{2}+\frac{\partial \widetilde{x}_{1}}{\partial x_{1}} \widetilde{P}_{0}+\frac{\widetilde{x}_{2}}{3} \frac{\partial \widetilde{x}_{2}}{\partial x_{1}}\right) .
$$

Using this relation, (2.33) and (2.34), we have

$$
\begin{aligned}
0= & \left(P_{0}^{3}+a_{2}(x) P_{0}+a_{3}(x)\right) T_{0} \\
= & T_{0}\left(\frac{\partial \widetilde{x}_{2}}{\partial x_{1}} \widetilde{P}_{0}^{2}+\frac{\partial \widetilde{x}_{1}}{\partial x_{1}} \widetilde{P}_{0}+\frac{\widetilde{x}_{2}}{3} \frac{\partial \widetilde{x}_{2}}{\partial x_{1}}\right)^{3}+a_{2}(x) T_{0}\left(\frac{\partial \widetilde{x}_{2}}{\partial x_{1}} \widetilde{P}_{0}^{2}+\frac{\partial \widetilde{x}_{1}}{\partial x_{1}} \widetilde{P}_{0}+\frac{\widetilde{x}_{2}}{3} \frac{\partial \widetilde{x}_{2}}{\partial x_{1}}\right) \\
= & T_{0}\left[\left\{\frac{\widetilde{x}_{2}^{2}}{12}\left(\frac{\partial \widetilde{x}_{2}}{\partial x_{1}}\right)^{3}-\frac{3}{4} \widetilde{x}_{1} \frac{\partial \widetilde{x}_{1}}{\partial x_{1}}\left(\frac{\partial \widetilde{x}_{2}}{\partial x_{1}}\right)^{2}-\frac{\widetilde{x}_{2}}{2}\left(\frac{\partial \widetilde{x}_{1}}{\partial x_{1}}\right)^{2} \frac{\partial \widetilde{x}_{2}}{\partial x_{1}}+a_{2}(x) \frac{\partial \widetilde{x}_{2}}{\partial x_{1}}\right\} \widetilde{P}_{0}^{2}\right. \\
& +\left\{\frac{\widetilde{x}_{2}^{2}}{12} \frac{\partial \widetilde{x}_{1}}{\partial x_{1}}\left(\frac{\partial \widetilde{x}_{2}}{\partial x_{1}}\right)^{2}-\frac{3}{4} \widetilde{x}_{1}\left(\frac{\partial \widetilde{x}_{1}}{\partial x_{1}}\right)^{2} \frac{\partial \widetilde{x}_{2}}{\partial x_{1}}-\frac{\widetilde{x}_{2}}{2}\left(\frac{\partial \widetilde{x}_{1}}{\partial x_{1}}\right)^{3}+a_{2}(x) \frac{\partial \widetilde{x}_{1}}{\partial x_{1}}\right\} \widetilde{P}_{0} \\
& \left.+\left\{\left(\frac{\widetilde{x}_{1}^{2}}{16}+\frac{\widetilde{x}_{2}^{3}}{27}\right)\left(\frac{\partial \widetilde{x}_{2}}{\partial x_{1}}\right)^{3}-\frac{\widetilde{x}_{1}}{4}\left(\frac{\partial \widetilde{x}_{1}}{\partial x_{1}}\right)^{3}-\frac{\widetilde{x}_{1} \widetilde{x}_{2}}{8} \frac{\partial \widetilde{x}_{1}}{\partial x_{1}}\left(\frac{\partial \widetilde{x}_{2}}{\partial x_{1}}\right)^{2}+a_{2}(x) \frac{\widetilde{x}_{2}}{3} \frac{\partial \widetilde{x}_{2}}{\partial x_{1}}+a_{3}(x)\right\}\right] .
\end{aligned}
$$

Hence, by the same reasoning as in the proof of Lemma 2.4, we get

$$
\begin{aligned}
& \frac{\partial \widetilde{x}_{2}}{\partial x_{1}}\left\{\frac{\widetilde{x}_{2}^{2}}{12}\left(\frac{\partial \widetilde{x}_{2}}{\partial x_{1}}\right)^{2}-\frac{3}{4} \widetilde{x}_{1} \frac{\partial \widetilde{x}_{1}}{\partial x_{1}} \frac{\partial \widetilde{x}_{2}}{\partial x_{1}}-\frac{\widetilde{x}_{2}}{2}\left(\frac{\partial \widetilde{x}_{1}}{\partial x_{1}}\right)^{2}+a_{2}(x)\right\}=0, \\
& \frac{\partial \widetilde{x}_{1}}{\partial x_{1}}\left\{\frac{\widetilde{x}_{2}^{2}}{12}\left(\frac{\partial \widetilde{x}_{2}}{\partial x_{1}}\right)^{2}-\frac{3}{4} \widetilde{x}_{1} \frac{\partial \widetilde{x}_{1}}{\partial x_{1}} \frac{\partial \widetilde{x}_{2}}{\partial x_{1}}-\frac{\widetilde{x}_{2}}{2}\left(\frac{\partial \widetilde{x}_{1}}{\partial x_{1}}\right)^{2}+a_{2}(x)\right\}=0,
\end{aligned}
$$




$$
\begin{aligned}
\left(\frac{\widetilde{x}_{1}^{2}}{16}+\frac{\widetilde{x}_{2}^{3}}{27}\right)\left(\frac{\partial \widetilde{x}_{2}}{\partial x_{1}}\right)^{3}-\frac{\widetilde{x}_{1}}{4}\left(\frac{\partial \widetilde{x}_{1}}{\partial x_{1}}\right)^{3}- & \frac{\widetilde{x}_{1} \widetilde{x}_{2}}{8} \frac{\partial \widetilde{x}_{1}}{\partial x_{1}}\left(\frac{\partial \widetilde{x}_{2}}{\partial x_{1}}\right)^{2} \\
& +a_{2}(x) \frac{\widetilde{x}_{2}}{3} \frac{\partial \widetilde{x}_{2}}{\partial x_{1}}+a_{3}(x)=0 .
\end{aligned}
$$

Here, if $\left(\partial \widetilde{x}_{1} / \partial x_{1}\right)(0)$ and $\left(\partial \widetilde{x}_{2} / \partial x_{1}\right)(0)$ simultaneously vanish, then $\left(\partial a_{3} / \partial x_{1}\right)(0)$ $=0$ by the assumption $a_{2}(0)=0$ and (4.12). This contradicts the assumption, that is, $\left(\partial \widetilde{x}_{1} / \partial x_{1}\right) \neq 0$ or $\left(\partial \widetilde{x}_{2} / \partial x_{1}\right) \neq 0$ holds near $x=0$. Hence we get the first equation of (4.8) by (4.10) and (4.11). Furthermore, using the first equation of (4.8) and (4.12), we obtain the second equation of (4.8).

Next, using (2.29), (2.34) and (4.9), we obtain

$$
\begin{aligned}
Q_{0} T_{0}= & \left(b_{2}(x) P_{0}^{2}+b_{1}(x) P_{0}+b_{0}(x)\right) T_{0} \\
= & b_{2}(x) T_{0}\left(\frac{\partial \widetilde{x}_{2}}{\partial x_{1}} \widetilde{P}_{0}^{2}+\frac{\partial \widetilde{x}_{1}}{\partial x_{1}} \widetilde{P}_{0}+\frac{\widetilde{x}_{2}}{3} \frac{\partial \widetilde{x}_{2}}{\partial x_{1}}\right)^{2} \\
& +b_{1}(x) T_{0}\left(\frac{\partial \widetilde{x}_{2}}{\partial x_{1}} \widetilde{P}_{0}^{2}+\frac{\partial \widetilde{x}_{1}}{\partial x_{1}} \widetilde{P}_{0}+\frac{\widetilde{x}_{2}}{3} \frac{\partial \widetilde{x}_{2}}{\partial x_{1}}\right)+b_{0}(x) T_{0} \\
= & T_{0}\left(\left[b_{2}(x)\left\{\left(\frac{\partial \widetilde{x}_{1}}{\partial x_{1}}\right)^{2}+\frac{\widetilde{x}_{2}}{6}\left(\frac{\partial \widetilde{x}_{2}}{\partial x_{1}}\right)^{2}\right\}+b_{1}(x) \frac{\partial \widetilde{x}_{2}}{\partial x_{1}}\right] \widetilde{P}_{0}^{2}\right. \\
& +\left[b_{2}(x)\left\{-\frac{\widetilde{x}_{2}}{3} \frac{\partial \widetilde{x}_{1}}{\partial x_{1}} \frac{\partial \widetilde{x}_{2}}{\partial x_{1}}-\frac{\widetilde{x}_{1}}{4}\left(\frac{\partial \widetilde{x}_{2}}{\partial x_{1}}\right)^{2}\right\}+b_{1}(x) \frac{\partial \widetilde{x}_{1}}{\partial x_{1}}\right] \widetilde{P}_{0} \\
& \left.+\left[b_{2}(x)\left\{\frac{\widetilde{x}_{2}^{2}}{9}\left(\frac{\partial \widetilde{x}_{2}}{\partial x_{1}}\right)^{2}-\frac{\widetilde{x}_{1}}{2} \frac{\partial \widetilde{x}_{1}}{\partial x_{1}} \frac{\partial \widetilde{x}_{2}}{\partial x_{1}}\right\}+b_{1}(x) \frac{\widetilde{x}_{2}}{3} \frac{\partial \widetilde{x}_{2}}{\partial x_{1}}+b_{0}(x)\right]\right) .
\end{aligned}
$$

On the other hand, the left-hand side of this equation can be expressed as

$$
Q_{0} T_{0}=T_{0}\left(\frac{\partial \widetilde{x}_{2}}{\partial x_{2}} \widetilde{P}_{0}^{2}+\frac{\partial \widetilde{x}_{1}}{\partial x_{2}} \widetilde{P}_{0}+\frac{\widetilde{x}_{2}}{3} \frac{\partial \widetilde{x}_{2}}{\partial x_{2}}\right)
$$

in view of (4.3) and the second equation of (4.5). Hence, we get

$$
\begin{aligned}
\frac{\partial \widetilde{x}_{2}}{\partial x_{2}} & =b_{2}(x)\left\{\left(\frac{\partial \widetilde{x}_{1}}{\partial x_{1}}\right)^{2}+\frac{\widetilde{x}_{2}}{6}\left(\frac{\partial \widetilde{x}_{2}}{\partial x_{1}}\right)^{2}\right\}+b_{1}(x) \frac{\partial \widetilde{x}_{2}}{\partial x_{1}} \\
\frac{\partial \widetilde{x}_{1}}{\partial x_{2}} & =b_{2}(x)\left\{-\frac{\widetilde{x}_{2}}{3} \frac{\partial \widetilde{x}_{1}}{\partial x_{1}} \frac{\partial \widetilde{x}_{2}}{\partial x_{1}}-\frac{\widetilde{x}_{1}}{4}\left(\frac{\partial \widetilde{x}_{2}}{\partial x_{1}}\right)^{2}\right\}+b_{1}(x) \frac{\partial \widetilde{x}_{1}}{\partial x_{1}} \\
\frac{\widetilde{x}_{2}}{3} \frac{\partial \widetilde{x}_{2}}{\partial x_{2}} & =b_{2}(x)\left\{\frac{\widetilde{x}_{2}^{2}}{9}\left(\frac{\partial \widetilde{x}_{2}}{\partial x_{1}}\right)^{2}-\frac{\widetilde{x}_{1}}{2} \frac{\partial \widetilde{x}_{1}}{\partial x_{1}} \frac{\partial \widetilde{x}_{2}}{\partial x_{1}}\right\}+b_{1}(x) \frac{\widetilde{x}_{2}}{3} \frac{\partial \widetilde{x}_{2}}{\partial x_{1}}+b_{0}(x) .
\end{aligned}
$$

Thus we get the third and the fourth equations of (4.8).

Finally, we show that (4.16) follows from (4.8). Using the fourth equation of (4.8), we find that (4.16) is equivalent to

$$
b_{2}(x)\left\{\frac{\widetilde{x}_{2}^{2}}{18}\left(\frac{\partial \widetilde{x}_{2}}{\partial x_{1}}\right)^{2}-\frac{\widetilde{x}_{1}}{2} \frac{\partial \widetilde{x}_{1}}{\partial x_{1}} \frac{\partial \widetilde{x}_{2}}{\partial x_{1}}-\frac{\widetilde{x}_{2}}{3}\left(\frac{\partial \widetilde{x}_{1}}{\partial x_{1}}\right)^{2}\right\}+b_{0}(x)=0 .
$$


On the other hand, Lemmas 2.3 and 2.4 imply

$$
\begin{aligned}
0 & =\operatorname{tr} Q_{0}=\operatorname{tr}\left(b_{2}(x) P_{0}^{2}+b_{1}(x) P_{0}+b_{0}(0)\right) \\
& =\operatorname{tr}\left[b_{2}(x)\left\{\left(T^{\dagger}\right)^{-1} P_{0} T^{\dagger}\right\}^{2}+b_{1}(x)\left(T^{\dagger}\right)^{-1} P_{0} T^{\dagger}+b_{0}(0)\right] \\
& =-2 b_{2}(x) a_{2}(x)+3 b_{0}(x) .
\end{aligned}
$$

Hence (4.17) is equivalent also to

$$
b_{2}(x)\left\{\frac{\widetilde{x}_{2}^{2}}{18}\left(\frac{\partial \widetilde{x}_{2}}{\partial x_{1}}\right)^{2}-\frac{\widetilde{x}_{1}}{2} \frac{\partial \widetilde{x}_{1}}{\partial x_{1}} \frac{\partial \widetilde{x}_{2}}{\partial x_{1}}-\frac{\widetilde{x}_{2}}{3}\left(\frac{\partial \widetilde{x}_{1}}{\partial x_{1}}\right)^{2}+\frac{2}{3} a_{2}(x)\right\}=0 .
$$

This relation exactly coincides with the first equation of (4.8).

In what follows we discuss the existence of a holomorphic solution $\left(\widetilde{x}_{1}(x), \widetilde{x}_{2}(x)\right)$ of $(4.8)$ near $x=0$.

Let $F$ (resp., $G$ ) denote the left-hand side of the first (resp., second) equation of (4.8). We first prove

Lemma 4.3. Let us consider a system of ordinary differential equations

$$
\left\{\begin{array}{l}
\left.F\right|_{x_{2}=0}=0 \\
\left.G\right|_{x_{2}=0}=0
\end{array}\right.
$$

obtained by restricting $F=0$ and $G=0$ to $x_{2}=0$. Then this system has a holomorphic solution $\left(\widetilde{x}_{1}\left(x_{1}, 0\right), \widetilde{x}_{2}\left(x_{1}, 0\right)\right)$ near $x_{1}=0$ satisfying

$$
\widetilde{x}_{1}(0)=0, \quad \widetilde{x}_{2}(0)=0, \quad \frac{\partial \widetilde{x}_{1}}{\partial x_{1}}(0) \neq 0 .
$$

In the proof we use the following Briot-Bouquet type theorem for a system of ordinary differential equations, established by Kaneko and Ohyama [KO].

Theorem 4.4. Let $h(t, u, v)$ and $k(t, u, v)$ be holomorphic functions near $(t, u, v)$ $=0$ satisfying

- $h(0)=k(0)=0$.

- For any non-negative integer $n$,

$$
\operatorname{det}\left(\begin{array}{cc}
n-\frac{\partial h}{\partial u}(0) & -\frac{\partial h}{\partial v}(0) \\
-\frac{\partial k}{\partial u}(0) & n-\frac{\partial k}{\partial v}(0)
\end{array}\right) \neq 0 .
$$

Then the system of ordinary differential equations

$$
\left\{\begin{array}{l}
t \frac{d u}{d t}=h(t, u, v), \\
t \frac{d v}{d t}=k(t, u, v)
\end{array}\right.
$$

has a holomorphic solution $(u(t), v(t))$ near $t=0$ satisfying $u(0)=v(0)=0$. 
Proof of Lemma 4.3. Let $Y\left(x_{1}\right), \widetilde{Y}\left(x_{1}\right), Z\left(x_{1}\right)$ and $\widetilde{Z}\left(x_{1}\right)$ be holomorphic functions defined by

$$
\left.\widetilde{x}_{1}\right|_{x_{2}=0}=Y=x_{1} \tilde{Y},\left.\quad \widetilde{x}_{2}\right|_{x_{2}=0}=Z=x_{1} \tilde{Z} .
$$

By the assumption $a_{2}(0)=a_{3}(0)=0$, we have

$$
\left.a_{2}\right|_{x_{2}=0}=\sum_{n \geq 1} a_{2, n} x_{1}^{n},\left.\quad a_{3}\right|_{x_{2}=0}=\sum_{n \geq 1} a_{3, n} x_{1}^{n} .
$$

Using these symbols, we can write (4.19) as

$$
\left\{\begin{aligned}
f\left(x_{1}, \widetilde{Y}, \widetilde{Z}, Y_{1}, Z_{1}\right):= & -\frac{1}{2} \widetilde{Z} Y_{1}^{2}-\frac{3}{4} \widetilde{Y} Y_{1} Z_{1}+\frac{x_{1}}{12} \widetilde{Z}^{2} Z_{1}^{2}+\sum_{n \geq 1} a_{2, n} x_{1}^{n-1}=0 \\
g\left(x_{1}, \widetilde{Y}, \widetilde{Z}, Y_{1}, Z_{1}\right):= & -\frac{1}{4} \widetilde{Y} Y_{1}^{3}+\frac{x_{1}}{6} \widetilde{Z}^{2} Y_{1}^{2} Z_{1}+\frac{x_{1}}{8} \widetilde{Y} \widetilde{Z} Y_{1} Z_{1}^{2} \\
& +\frac{x_{1}}{16} \widetilde{Y}^{2} Z_{1}^{3}+\frac{x_{1}^{2}}{108} \widetilde{Z}^{3} Z_{1}^{3}+\sum_{n \geq 1} a_{3, n} x_{1}^{n-1} \\
= & 0
\end{aligned}\right.
$$

where $Y_{1}=d Y / d x_{1}$ and $Z_{1}=d Z / d x_{1}$.

First, by the assumption $\left(\partial a_{3} / \partial x_{1}\right)(0)=a_{3,1} \neq 0$, a solution of

$$
\left\{\begin{array}{l}
f(0, p, q, p, q)=0 \\
g(0, p, q, p, q)=0
\end{array}\right.
$$

is given by

$$
p=\left(4 a_{3,1}\right)^{1 / 4} \neq 0, \quad q=\frac{2 a_{2,1}}{5\left(a_{3,1}\right)^{1 / 2}} .
$$

Using this fact, we have

$$
\left.\operatorname{det}\left(\begin{array}{cc}
\frac{\partial f}{\partial Y_{1}} & \frac{\partial f}{\partial Z_{1}} \\
\frac{\partial g}{\partial Y_{1}} & \frac{\partial g}{\partial Z_{1}}
\end{array}\right)\right|_{\left(x_{1}, \widetilde{Y}, \widetilde{Z}, Y_{1}, Z_{1}\right)=(0, p, q, p, q)}=-\frac{9}{16} p^{5} \neq 0
$$

Then, thanks to the implicit function theorem, we can rewrite (4.23) as

$$
\left\{\begin{array}{l}
Y_{1}=H\left(x_{1}, \widetilde{Y}, \widetilde{Z}\right) \\
Z_{1}=K\left(x_{1}, \widetilde{Y}, \widetilde{Z}\right)
\end{array}\right.
$$

with some holomorphic functions $H\left(x_{1}, \tilde{Y}, \widetilde{Z}\right), K\left(x_{1}, \tilde{Y}, \widetilde{Z}\right)$ near $\left(x_{1}, \tilde{Y}, \widetilde{Z}\right)=$ $(0, p, q)$. Introducing new unknown functions $\widehat{Y}=\widetilde{Y}-p$ and $\widehat{Z}=\widetilde{Z}-q$, we further rewrite (4.24) as 


$$
\left\{\begin{array}{l}
x_{1} \frac{d \widehat{Y}}{d x_{1}}=H\left(x_{1}, p+\widehat{Y}, q+\widehat{Z}\right)-p-\widehat{Y}=: \widehat{H}\left(x_{1}, \widehat{Y}, \widehat{Z}\right), \\
x_{1} \frac{d \widehat{Z}}{d x_{1}}=K\left(x_{1}, p+\widehat{Y}, q+\widehat{Z}\right)-q-\widehat{Z}=: \widehat{K}\left(x_{1}, \widehat{Y}, \widehat{Z}\right),
\end{array}\right.
$$

where $\widehat{H}\left(x_{1}, \widehat{Y}, \widehat{Z}\right)$ and $\widehat{K}\left(x_{1}, \widehat{Y}, \widehat{Z}\right)$ are holomorphic functions near $\left(x_{1}, \widehat{Y}, \widehat{Z}\right)=0$. Substituting these relations into (4.23), we have

$$
\begin{array}{lll}
\widehat{H}(0)=0, & \frac{\partial \widehat{H}}{\partial \widehat{Y}}(0)=-\frac{4}{3}, & \frac{\partial \widehat{H}}{\partial \widehat{Z}}(0)=0, \\
\widehat{K}(0)=0, & \frac{\partial \widehat{K}}{\partial \widehat{Y}}(0)=-\frac{2 q}{9 p}, & \frac{\partial \widehat{K}}{\partial \widehat{Z}}(0)=-\frac{5}{3} .
\end{array}
$$

This condition means that (4.25) satisfies the assumptions of Theorem 4.4. Hence, applying Theorem 4.4, we find that (4.25) has a holomorphic solution $\left(\widehat{Y}\left(x_{1}\right), \widehat{Z}\left(x_{1}\right)\right)$ with $\widehat{Y}(0)=\widehat{Z}(0)=0$, that is, (4.19) has a holomorphic solution $\left(\widetilde{x}_{1}\left(x_{1}, 0\right), \widetilde{x}_{2}\left(x_{1}, 0\right)\right)$ near $x_{1}=0$. The construction of the solution also satisfies

$$
\widetilde{x}_{1}\left(x_{1}, 0\right)=x_{1}\left(p+\mathcal{O}\left(x_{1}\right)\right), \quad \widetilde{x}_{2}\left(x_{1}, 0\right)=x_{1}\left(q+\mathcal{O}\left(x_{1}\right)\right),
$$

that is, (4.20) holds.

For this solution $\left(\widetilde{x}_{1}\left(x_{1}, 0\right), \widetilde{x}_{2}\left(x_{1}, 0\right)\right)$, we have

Lemma 4.5. Let $\left(\widetilde{x}_{1}\left(x_{1}, 0\right), \widetilde{x}_{2}\left(x_{1}, 0\right)\right)$ be the holomorphic solution of (4.19) near $x_{1}=0$ obtained in Lemma 4.3. Then the initial value problem

$$
\left\{\begin{array}{l}
\frac{\partial \widetilde{x}_{1}}{\partial x_{2}}=b_{2}(x)\left\{-\frac{\widetilde{x}_{2}}{3} \frac{\partial \widetilde{x}_{1}}{\partial x_{1}} \frac{\partial \widetilde{x}_{2}}{\partial x_{1}}-\frac{\widetilde{x}_{1}}{4}\left(\frac{\partial \widetilde{x}_{2}}{\partial x_{1}}\right)^{2}\right\}+b_{1}(x) \frac{\partial \widetilde{x}_{1}}{\partial x_{1}} \\
\frac{\partial \widetilde{x}_{2}}{\partial x_{2}}=b_{2}(x)\left\{\left(\frac{\partial \widetilde{x}_{1}}{\partial x_{1}}\right)^{2}+\frac{\widetilde{x}_{2}}{6}\left(\frac{\partial \widetilde{x}_{2}}{\partial x_{1}}\right)^{2}\right\}+b_{1}(x) \frac{\partial \widetilde{x}_{2}}{\partial x_{1}} \\
\widetilde{x}_{\left.\right|_{x_{2}}=0}=\widetilde{x}_{i}\left(x_{1}, 0\right) \quad(i=1,2)
\end{array}\right.
$$

has a holomorphic solution near $x=0$.

This lemma is an immediate consequence of the Cauchy-Kowalevski theorem.

Lemma 4.6. The holomorphic solution $\left(\widetilde{x}_{1}(x), \widetilde{x}_{2}(x)\right)$ given by Lemma 4.5 satisfies

$$
F=G=0 .
$$

Proof. For simplicity, in this proof $\left.F\right|_{\left(\widetilde{x}_{1}, \widetilde{x}_{2}\right)=\left(\widetilde{x}_{1}(x), \widetilde{x}_{2}(x)\right)}$ and $\left.G\right|_{\left(\widetilde{x}_{1}, \widetilde{x}_{2}\right)=\left(\widetilde{x}_{1}(x), \widetilde{x}_{2}(x)\right)}$ are denoted by $F$ and $G$, respectively. We prove that $F$ and $G$ satisfy the system of partial differential equations 


$$
\left\{\begin{array}{l}
\frac{\partial F}{\partial x_{2}}=2 b_{2} \frac{\partial G}{\partial x_{1}}+b_{1} \frac{\partial F}{\partial x_{1}}+3 \frac{\partial b_{2}}{\partial x_{1}} G+2 \frac{\partial b_{1}}{\partial x_{1}} F \\
\frac{\partial G}{\partial x_{2}}=b_{1} \frac{\partial G}{\partial x_{1}}+\frac{1}{3} b_{2} \frac{\partial}{\partial x_{1}} F\left(F-2 a_{2}\right)+3 \frac{\partial b_{1}}{\partial x_{1}} G+\frac{2}{3} \frac{\partial b_{2}}{\partial x_{1}} F\left(F-2 a_{2}\right) .
\end{array}\right.
$$

Using the definition of $F$ and $G$, we have

$$
\begin{aligned}
& \frac{\partial}{\partial x_{1}}\left(F-a_{2}\right)=\left\{\frac{\widetilde{x}_{2}}{6}\left(\frac{\partial \widetilde{x}_{2}}{\partial x_{1}}\right)^{3}-\frac{5}{4}\left(\frac{\partial \widetilde{x}_{1}}{\partial x_{1}}\right)^{2} \frac{\partial \widetilde{x}_{2}}{\partial x_{1}}\right\}+\left(-\frac{3}{4} \widetilde{x}_{1} \frac{\partial \widetilde{x}_{2}}{\partial x_{1}}-\widetilde{x}_{2} \frac{\partial \widetilde{x}_{1}}{\partial x_{1}}\right) \frac{\partial^{2} \widetilde{x}_{1}}{\partial x_{1}^{2}} \\
& +\left(\frac{\widetilde{x}_{2}^{2}}{6} \frac{\partial \widetilde{x}_{2}}{\partial x_{1}}-\frac{3}{4} \widetilde{x}_{1} \frac{\partial \widetilde{x}_{1}}{\partial x_{1}}\right) \frac{\partial^{2} \widetilde{x}_{2}}{\partial x_{1}^{2}}, \\
& \frac{\partial}{\partial x_{1}}\left(G-a_{3}\right) \\
& =\left\{\frac{\widetilde{x}_{1}}{4} \frac{\partial \widetilde{x}_{1}}{\partial x_{1}}\left(\frac{\partial \widetilde{x}_{2}}{\partial x_{1}}\right)^{3}+\frac{\widetilde{x}_{2}^{2}}{36}\left(\frac{\partial \widetilde{x}_{2}}{\partial x_{1}}\right)^{4}-\frac{1}{4}\left(\frac{\partial \widetilde{x}_{1}}{\partial x_{1}}\right)^{4}+\frac{11}{24} \widetilde{x}_{2}\left(\frac{\partial \widetilde{x}_{1}}{\partial x_{1}}\right)^{2}\left(\frac{\partial \widetilde{x}_{2}}{\partial x_{1}}\right)^{2}\right\} \\
& +\left\{-\frac{3}{4} \widetilde{x}_{1}\left(\frac{\partial \widetilde{x}_{1}}{\partial x_{1}}\right)^{2}+\frac{\widetilde{x}_{1} \widetilde{x}_{2}}{8}\left(\frac{\partial \widetilde{x}_{2}}{\partial x_{1}}\right)^{2}+\frac{\widetilde{x}_{2}^{2}}{3} \frac{\partial \widetilde{x}_{1}}{\partial x_{1}} \frac{\partial \widetilde{x}_{2}}{\partial x_{1}}\right\} \frac{\partial^{2} \widetilde{x}_{1}}{\partial x_{1}^{2}} \\
& +\left\{\frac{3}{16} \widetilde{x}_{1}^{2}\left(\frac{\partial \widetilde{x}_{2}}{\partial x_{1}}\right)^{2}+\frac{\widetilde{x}_{2}^{3}}{36}\left(\frac{\partial \widetilde{x}_{2}}{\partial x_{1}}\right)^{2}+\frac{\widetilde{x}_{1} \widetilde{x}_{2}}{4} \frac{\partial \widetilde{x}_{1}}{\partial x_{1}} \frac{\partial \widetilde{x}_{2}}{\partial x_{1}}+\frac{\widetilde{x}_{2}^{2}}{6}\left(\frac{\partial \widetilde{x}_{1}}{\partial x_{1}}\right)^{2}\right\} \frac{\partial^{2} \widetilde{x}_{2}}{\partial x_{1}^{2}}, \\
& \frac{\partial}{\partial x_{2}}\left(F-a_{2}\right)=\left(-\frac{3}{4} \frac{\partial \widetilde{x}_{1}}{\partial x_{1}} \frac{\partial \widetilde{x}_{2}}{\partial x_{1}}\right) \frac{\partial \widetilde{x}_{1}}{\partial x_{2}}+\left\{\frac{\widetilde{x}_{2}}{6}\left(\frac{\partial \widetilde{x}_{2}}{\partial x_{1}}\right)^{2}-\frac{1}{2}\left(\frac{\partial \widetilde{x}_{1}}{\partial x_{1}}\right)^{2}\right\} \frac{\partial \widetilde{x}_{2}}{\partial x_{2}} \\
& +\left(-\frac{3}{4} \widetilde{x}_{1} \frac{\partial \widetilde{x}_{2}}{\partial x_{1}}-\widetilde{x}_{2} \frac{\partial \widetilde{x}_{1}}{\partial x_{1}}\right) \frac{\partial^{2} \widetilde{x}_{1}}{\partial x_{1} \partial x_{2}}+\left(\frac{\widetilde{x}_{2}^{2}}{6} \frac{\partial \widetilde{x}_{2}}{\partial x_{1}}-\frac{3}{4} \widetilde{x}_{1} \frac{\partial \widetilde{x}_{1}}{\partial x_{1}}\right) \frac{\partial^{2} \widetilde{x}_{2}}{\partial x_{1} \partial x_{2}}, \\
& \frac{\partial}{\partial x_{2}}\left(G-a_{3}\right)=\left\{\frac{\widetilde{x}_{1}}{8}\left(\frac{\partial \widetilde{x}_{2}}{\partial x_{1}}\right)^{3}-\frac{1}{4}\left(\frac{\partial \widetilde{x}_{1}}{\partial x_{1}}\right)^{3}+\frac{\widetilde{x}_{2}}{8} \frac{\partial \widetilde{x}_{1}}{\partial x_{1}}\left(\frac{\partial \widetilde{x}_{2}}{\partial x_{1}}\right)^{2}\right\} \frac{\partial \widetilde{x}_{1}}{\partial x_{2}} \\
& +\left\{\frac{\widetilde{x}_{2}^{2}}{36}\left(\frac{\partial \widetilde{x}_{2}}{\partial x_{1}}\right)^{3}+\frac{\widetilde{x}_{1}}{8} \frac{\partial \widetilde{x}_{1}}{\partial x_{1}}\left(\frac{\partial \widetilde{x}_{2}}{\partial x_{1}}\right)^{2}+\frac{\widetilde{x}_{2}}{3}\left(\frac{\partial \widetilde{x}_{1}}{\partial x_{1}}\right)^{2} \frac{\partial \widetilde{x}_{2}}{\partial x_{1}}\right\} \frac{\partial \widetilde{x}_{2}}{\partial x_{2}} \\
& +\left\{-\frac{3}{4} \widetilde{x}_{1}\left(\frac{\partial \widetilde{x}_{1}}{\partial x_{1}}\right)^{2}+\frac{\widetilde{x}_{1} \widetilde{x}_{2}}{8}\left(\frac{\partial \widetilde{x}_{2}}{\partial x_{1}}\right)^{2}+\frac{\widetilde{x}_{2}^{2}}{3} \frac{\partial \widetilde{x}_{1}}{\partial x_{1}} \frac{\partial \widetilde{x}_{2}}{\partial x_{1}}\right\} \frac{\partial^{2} \widetilde{x}_{1}}{\partial x_{1} \partial x_{2}} \\
& +\left\{\frac{3}{16} \widetilde{x}_{1}^{2}\left(\frac{\partial \widetilde{x}_{2}}{\partial x_{1}}\right)^{2}+\frac{\widetilde{x}_{2}^{3}}{36}\left(\frac{\partial \widetilde{x}_{2}}{\partial x_{1}}\right)^{2}+\frac{\widetilde{x}_{1} \widetilde{x}_{2}}{4} \frac{\partial \widetilde{x}_{1}}{\partial x_{1}} \frac{\partial \widetilde{x}_{2}}{\partial x_{1}}+\frac{\widetilde{x}_{2}^{2}}{6}\left(\frac{\partial \widetilde{x}_{1}}{\partial x_{1}}\right)^{2}\right\} \frac{\partial^{2} \widetilde{x}_{2}}{\partial x_{1} \partial x_{2}} .
\end{aligned}
$$

Here, by (4.26), we find

$$
\begin{aligned}
& \frac{\partial^{2} \widetilde{x}_{1}}{\partial x_{1} \partial x_{2}}=\frac{\partial b_{2}}{\partial x_{1}}(x)\left\{-\frac{\widetilde{x}_{2}}{3} \frac{\partial \widetilde{x}_{1}}{\partial x_{1}} \frac{\partial \widetilde{x}_{2}}{\partial x_{1}}-\frac{\widetilde{x}_{1}}{4}\left(\frac{\partial \widetilde{x}_{2}}{\partial x_{1}}\right)^{2}\right\} \\
& \quad+b_{2}(x)\left\{-\frac{\widetilde{x}_{2}}{3} \frac{\partial \widetilde{x}_{2}}{\partial x_{1}} \frac{\partial^{2} \widetilde{x}_{1}}{\partial x_{1}^{2}}-\frac{7}{12} \frac{\partial \widetilde{x}_{1}}{\partial x_{1}}\left(\frac{\partial \widetilde{x}_{2}}{\partial x_{1}}\right)^{2}-\frac{\widetilde{x}_{2}}{3} \frac{\partial \widetilde{x}_{1}}{\partial x_{1}} \frac{\partial^{2} \widetilde{x}_{2}}{\partial x_{1}^{2}}-\frac{\widetilde{x}_{1}}{2} \frac{\partial \widetilde{x}_{2}}{\partial x_{1}} \frac{\partial^{2} \widetilde{x}_{2}}{\partial x_{1}^{2}}\right\} \\
& \quad+\frac{\partial b_{1}}{\partial x_{1}}(x) \frac{\partial \widetilde{x}_{1}}{\partial x_{1}}+b_{1}(x) \frac{\partial^{2} \widetilde{x}_{1}}{\partial x_{1}^{2}},
\end{aligned}
$$




$$
\begin{aligned}
& \frac{\partial^{2} \widetilde{x}_{2}}{\partial x_{1} \partial x_{2}}=\frac{\partial b_{2}}{\partial x_{1}}(x)\left\{\left(\frac{\partial \widetilde{x}_{1}}{\partial x_{1}}\right)^{2}+\frac{\widetilde{x}_{2}}{6}\left(\frac{\partial \widetilde{x}_{2}}{\partial x_{1}}\right)^{2}\right\} \\
& \quad+b_{2}(x)\left\{2 \frac{\partial \widetilde{x}_{1}}{\partial x_{1}} \frac{\partial^{2} \widetilde{x}_{1}}{\partial x_{1}^{2}}+\frac{1}{6}\left(\frac{\partial \widetilde{x}_{2}}{\partial x_{1}}\right)^{3}+\frac{\widetilde{x}_{2}}{3} \frac{\partial \widetilde{x}_{2}}{\partial x_{1}} \frac{\partial^{2} \widetilde{x}_{2}}{\partial x_{1}^{2}}\right\}+\frac{\partial b_{1}}{\partial x_{1}}(x) \frac{\partial \widetilde{x}_{2}}{\partial x_{1}}+b_{1}(x) \frac{\partial^{2} \widetilde{x}_{2}}{\partial x_{1}^{2}} .
\end{aligned}
$$

Then, using these relations, we can confirm by a straightforward computation that

$$
\left\{\begin{array}{c}
\frac{\partial}{\partial x_{2}}\left(F-a_{2}\right)-2 b_{2} \frac{\partial}{\partial x_{1}}\left(G-a_{3}\right)-b_{1} \frac{\partial}{\partial x_{1}}\left(F-a_{2}\right) \\
-3 \frac{\partial b_{2}}{\partial x_{1}}\left(G-a_{3}\right)-2 \frac{\partial b_{1}}{\partial x_{1}}\left(F-a_{2}\right)=0 \\
\frac{\partial}{\partial x_{2}}\left(G-a_{3}\right)-b_{1} \frac{\partial}{\partial x_{1}}\left(G-a_{3}\right)-\frac{1}{3} b_{2} \frac{\partial}{\partial x_{1}}\left(F-a_{2}\right)^{2} \\
-3 \frac{\partial b_{1}}{\partial x_{1}}\left(G-a_{3}\right)-\frac{2}{3} \frac{\partial b_{2}}{\partial x_{1}}\left(F-a_{2}\right)^{2}=0
\end{array}\right.
$$

In view of (2.38) and (4.18), (4.28) immediately follows from (4.29).

On the other hand, by the construction of $\left(\widetilde{x}_{1}(x), \widetilde{x}_{2}(x)\right)$,

$$
\left.F\right|_{x_{2}=0}=\left.G\right|_{x_{2}=0}=0 .
$$

Hence, by the uniqueness of solutions for the initial value problem (4.28) and (4.30), we obtain (4.27).

We have thus confirmed that the system (4.8) has a holomorphic solution $\left(\widetilde{x}_{1}(x), \widetilde{x}_{2}(x)\right)$ near $x=0$. This solution has the following properties.

Lemma 4.7. Let $\left(\widetilde{x}_{1}(x), \widetilde{x}_{2}(x)\right)$ be the solution given by the above argument. Then

- $\widetilde{x}_{1}(0)=\widetilde{x}_{2}(0)=0$.

- The Jacobian

$$
\left|J_{\widetilde{x}}\right|(x)=\operatorname{det}\left(\begin{array}{ll}
\frac{\partial \widetilde{x}_{1}}{\partial x_{1}}(x) & \frac{\partial \widetilde{x}_{1}}{\partial x_{2}}(x) \\
\frac{\partial \widetilde{x}_{2}}{\partial x_{1}}(x) & \frac{\partial \widetilde{x}_{2}}{\partial x_{2}}(x)
\end{array}\right)
$$

of $\widetilde{x}(x)=\left(\widetilde{x}_{1}(x), \widetilde{x}_{2}(x)\right)$ is non-zero near $x=0$.

Proof. By Lemma 2.8 and the assumption $a_{2}(0)=a_{3}(0)=0$, we find

$$
\begin{aligned}
\operatorname{det}\left(\begin{array}{ll}
\frac{\partial a_{2}}{\partial x_{1}}(0) & \frac{\partial a_{2}}{\partial x_{2}}(0) \\
\frac{\partial a_{3}}{\partial x_{1}}(0) & \frac{\partial a_{3}}{\partial x_{2}}(0)
\end{array}\right) & =\operatorname{det}\left(\begin{array}{lc}
\frac{\partial a_{2}}{\partial x_{1}}(0) & 2 b_{2}(0) \frac{\partial a_{3}}{\partial x_{1}}(0)+b_{1}(0) \frac{\partial a_{2}}{\partial x_{1}}(0) \\
\frac{\partial a_{3}}{\partial x_{1}}(0) & b_{1}(0) \frac{\partial a_{3}}{\partial x_{1}}(0)
\end{array}\right) \\
& =-2 b_{2}(0)\left(\frac{\partial a_{3}}{\partial x_{1}}(0)\right)^{2}
\end{aligned}
$$


Hence, using the assumptions of Proposition 4.1, we get

$$
b_{2}(0) \neq 0 .
$$

It follows from the third and the fourth equations of (4.8) and (4.20) that

$$
\begin{aligned}
\frac{\partial \widetilde{x}_{1}}{\partial x_{2}}(0) & =b_{2}(0)\left\{-\frac{\widetilde{x}_{2}(0)}{3} \frac{\partial \widetilde{x}_{1}}{\partial x_{1}}(0) \frac{\partial \widetilde{x}_{2}}{\partial x_{1}}(0)-\frac{\widetilde{x}_{1}(0)}{4}\left(\frac{\partial \widetilde{x}_{2}}{\partial x_{1}}(0)\right)^{2}\right\}+b_{1}(0) \frac{\partial \widetilde{x}_{1}}{\partial x_{1}}(0) \\
& =b_{1}(0) \frac{\partial \widetilde{x}_{1}}{\partial x_{1}}(0), \\
\frac{\partial \widetilde{x}_{2}}{\partial x_{2}}(0) & =b_{2}(0)\left\{\left(\frac{\partial \widetilde{x}_{1}}{\partial x_{1}}(0)\right)^{2}+\frac{\widetilde{x}_{2}(0)}{6}\left(\frac{\partial \widetilde{x}_{2}}{\partial x_{1}}(0)\right)^{2}\right\}+b_{1}(0) \frac{\partial \widetilde{x}_{2}}{\partial x_{1}}(0) \\
& =b_{2}(0)\left(\frac{\partial \widetilde{x}_{1}}{\partial x_{1}}(0)\right)^{2}+b_{1}(0) \frac{\partial \widetilde{x}_{2}}{\partial x_{1}}(0) .
\end{aligned}
$$

We thus obtain

$$
\left|J_{\widetilde{x}}\right|(0)=b_{2}(0)\left(\frac{\partial \widetilde{x}_{1}}{\partial x_{1}}(0)\right)^{3} \neq 0,
$$

that is, $\left|J_{\widetilde{x}}\right|(x)$ is non-zero near $x=0$.

Next, we construct $T_{0}(x)$ which satisfies (4.5).

Lemma 4.8. There exists a $3 \times 3$ matrix $T_{0}(x)$ with holomorphic entries which is invertible near $x=0$ and satisfies

$$
T_{0}\left(\frac{\partial \widetilde{x}_{1}}{\partial x_{1}} \widetilde{P}_{0}+\frac{\partial \widetilde{x}_{2}}{\partial x_{1}} \widetilde{Q}_{0}\right)=P_{0} T_{0}
$$

Proof. Using (2.34), (4.3) and the first and the second equations of (4.8), we have

$$
\begin{aligned}
& \left(\frac{\partial \widetilde{x}_{1}}{\partial x_{1}} \widetilde{P}_{0}+\frac{\partial \widetilde{x}_{2}}{\partial x_{1}} \widetilde{Q}_{0}\right)^{3}+a_{2}(x)\left(\frac{\partial \widetilde{x}_{1}}{\partial x_{1}} \widetilde{P}_{0}+\frac{\partial \widetilde{x}_{2}}{\partial x_{1}} \widetilde{Q}_{0}\right)+a_{3}(x) \\
& =\left(\frac{\partial \widetilde{x}_{2}}{\partial x_{1}} \widetilde{P}_{0}^{2}+\frac{\partial \widetilde{x}_{1}}{\partial x_{1}} \widetilde{P}_{0}+\frac{\widetilde{x}_{2}}{3} \frac{\partial \widetilde{x}_{2}}{\partial x_{1}}\right)^{3}+a_{2}(x)\left(\frac{\partial \widetilde{x}_{2}}{\partial x_{1}} \widetilde{P}_{0}^{2}+\frac{\partial \widetilde{x}_{1}}{\partial x_{1}} \widetilde{P}_{0}+\frac{\widetilde{x}_{2}}{3} \frac{\partial \widetilde{x}_{2}}{\partial x_{1}}\right)+a_{3}(x) \\
& =\left\{\frac{\widetilde{x}_{2}^{2}}{12}\left(\frac{\partial \widetilde{x}_{2}}{\partial x_{1}}\right)^{3}-\frac{3}{4} \widetilde{x}_{1} \frac{\partial \widetilde{x}_{1}}{\partial x_{1}}\left(\frac{\partial \widetilde{x}_{2}}{\partial x_{1}}\right)^{2}-\frac{\widetilde{x}_{2}}{2}\left(\frac{\partial \widetilde{x}_{1}}{\partial x_{1}}\right)^{2} \frac{\partial \widetilde{x}_{2}}{\partial x_{1}}+a_{2}(x) \frac{\partial \widetilde{x}_{2}}{\partial x_{1}}\right\} \widetilde{P}_{0}^{2} \\
& +\left\{\frac{\widetilde{x}_{2}^{2}}{12} \frac{\partial \widetilde{x}_{1}}{\partial x_{1}}\left(\frac{\partial \widetilde{x}_{2}}{\partial x_{1}}\right)^{2}-\frac{3}{4} \widetilde{x}_{1}\left(\frac{\partial \widetilde{x}_{1}}{\partial x_{1}}\right)^{2} \frac{\partial \widetilde{x}_{2}}{\partial x_{1}}-\frac{\widetilde{x}_{2}}{2}\left(\frac{\partial \widetilde{x}_{1}}{\partial x_{1}}\right)^{3}+a_{2}(x) \frac{\partial \widetilde{x}_{1}}{\partial x_{1}}\right\} \widetilde{P}_{0} \\
& +\left\{\left(\frac{\widetilde{x}_{1}^{2}}{16}+\frac{\widetilde{x}_{2}^{3}}{27}\right)\left(\frac{\partial \widetilde{x}_{2}}{\partial x_{1}}\right)^{3}-\frac{\widetilde{x}_{1}}{4}\left(\frac{\partial \widetilde{x}_{1}}{\partial x_{1}}\right)^{3}-\frac{\widetilde{x}_{1} \widetilde{x}_{2}}{8} \frac{\partial \widetilde{x}_{1}}{\partial x_{1}}\left(\frac{\partial \widetilde{x}_{2}}{\partial x_{1}}\right)^{2}+\frac{a_{2}(x)}{3} \widetilde{x}_{2} \frac{\partial \widetilde{x}_{2}}{\partial x_{1}}+a_{3}(x)\right\} \\
& =0 .
\end{aligned}
$$


Then, by the same argument as in the proof of Lemma 2.3, we can find a $3 \times 3$ invertible matrix $\widetilde{T}^{\dagger}(x)$ with holomorphic entries near $x=0$ which satisfies

$$
\left(\widetilde{T}^{\dagger}\right)^{-1}\left(\frac{\partial \widetilde{x}_{1}}{\partial x_{1}} \widetilde{P}_{0}+\frac{\partial \widetilde{x}_{2}}{\partial x_{1}} \widetilde{Q}_{0}\right) \widetilde{T}^{\dagger}=\left(\begin{array}{ccc}
0 & 1 & 0 \\
0 & 0 & 1 \\
-a_{3} & -a_{2} & 0
\end{array}\right) .
$$

If we set $T_{0}(x)=T^{\dagger}(x) \widetilde{T}^{\dagger}(x)^{-1}$, where $T^{\dagger}(x)$ is the $3 \times 3$ matrix given by Lemma 2.3 , then $T_{0}(x)$ has the desired properties.

Furthermore, we have

\section{Lemma 4.9.}

$$
T_{0}\left(\frac{\partial \widetilde{x}_{1}}{\partial x_{2}} \widetilde{P}_{0}+\frac{\partial \widetilde{x}_{2}}{\partial x_{2}} \widetilde{Q}_{0}\right)=Q_{0} T_{0} .
$$

Proof. Using (2.29), (2.34), (4.3), the third and the fourth equations of (4.8), (4.9) and $(4.16)$, we obtain

$$
\begin{aligned}
Q_{0} T_{0}= & \left.b_{2}(x) P_{0}^{2}+b_{1}(x) P_{0}+b_{0}(x)\right) T_{0} \\
= & b_{2}(x) T_{0}\left(\frac{\partial \widetilde{x}_{2}}{\partial x_{1}} \widetilde{P}_{0}^{2}+\frac{\partial \widetilde{x}_{1}}{\partial x_{1}} \widetilde{P}_{0}+\frac{\widetilde{x}_{2}}{3} \frac{\partial \widetilde{x}_{2}}{\partial x_{1}}\right)^{2} \\
& +b_{1}(x) T_{0}\left(\frac{\partial \widetilde{x}_{2}}{\partial x_{1}} \widetilde{P}_{0}^{2}+\frac{\partial \widetilde{x}_{1}}{\partial x_{1}} \widetilde{P}_{0}+\frac{\widetilde{x}_{2}}{3} \frac{\partial \widetilde{x}_{2}}{\partial x_{1}}\right)+b_{0}(x) T_{0} \\
=T_{0} & \left(\left[b_{2}(x)\left\{\left(\frac{\partial \widetilde{x}_{1}}{\partial x_{1}}\right)^{2}+\frac{\widetilde{x}_{2}}{6}\left(\frac{\partial \widetilde{x}_{2}}{\partial x_{1}}\right)^{2}\right\}+b_{1}(x) \frac{\partial \widetilde{x}_{2}}{\partial x_{1}}\right] \widetilde{P}_{0}^{2}\right. \\
& +\left[b_{2}(x)\left\{-\frac{\widetilde{x}_{2}}{3} \frac{\partial \widetilde{x}_{1}}{\partial x_{1}} \frac{\partial \widetilde{x}_{2}}{\partial x_{1}}-\frac{\widetilde{x}_{1}}{4}\left(\frac{\partial \widetilde{x}_{2}}{\partial x_{1}}\right)^{2}\right\}+b_{1}(x) \frac{\partial \widetilde{x}_{1}}{\partial x_{1}}\right] \widetilde{P}_{0} \\
& \left.+\left[b_{2}(x)\left\{\frac{\widetilde{x}_{2}^{2}}{9}\left(\frac{\partial \widetilde{x}_{2}}{\partial x_{1}}\right)^{2}-\frac{\widetilde{x}_{1}}{2} \frac{\partial \widetilde{x}_{1}}{\partial x_{1}} \frac{\partial \widetilde{x}_{2}}{\partial x_{1}}\right\}+\frac{b_{1}(x)}{3} \widetilde{x}_{2} \frac{\partial \widetilde{x}_{2}}{\partial x_{1}}+b_{0}(x)\right]\right) \\
= & T_{0}\left(\frac{\partial \widetilde{x}_{2}}{\partial x_{2}} \widetilde{P}_{0}^{2}+\frac{\partial \widetilde{x}_{1}}{\partial x_{2}} \widetilde{P}_{0}+\frac{\widetilde{x}_{2}}{3} \frac{\partial \widetilde{x}_{2}}{\partial x_{2}}\right) \\
= & T_{0}\left(\frac{\partial \widetilde{x}_{1}}{\partial x_{2}} \widetilde{P}_{0}+\frac{\partial \widetilde{x}_{2}}{\partial x_{2}} \widetilde{Q}_{0}\right) .
\end{aligned}
$$

Using these lemmas, we now prove Proposition 4.1.

Proof of Proposition 4.1. Let $\left(\widetilde{x}_{1}(x), \widetilde{x}_{2}(x)\right)$ be a holomorphic solution of (4.8) constructed in Lemmas 4.3-4.7, and let $T_{0}(x)$ be a $3 \times 3$ invertible matrix with holomorphic entries given in Lemmas 4.8 and 4.9. Let us define $3 \times 3$ matrices $\widetilde{P}_{n}(x)$ and $\widetilde{Q}_{n}(x)$ by 


$$
\begin{aligned}
& \left\{\begin{array}{l}
\widetilde{P}_{1}=\left(\left|J_{\widetilde{x}}\right| T_{0}\right)^{-1}\left\{\frac{\partial \widetilde{x}_{2}}{\partial x_{2}}\left(P_{1} T_{0}-\frac{\partial T_{0}}{\partial x_{1}}\right)-\frac{\partial \widetilde{x}_{2}}{\partial x_{1}}\left(Q_{1} T_{0}-\frac{\partial T_{0}}{\partial x_{2}}\right)\right\} \\
\widetilde{Q}_{1}=\left(\left|J_{\widetilde{x}}\right| T_{0}\right)^{-1}\left\{\frac{\partial \widetilde{x}_{1}}{\partial x_{1}}\left(Q_{1} T_{0}-\frac{\partial T_{0}}{\partial x_{2}}\right)-\frac{\partial \widetilde{x}_{1}}{\partial x_{2}}\left(P_{1} T_{0}-\frac{\partial T_{0}}{\partial x_{1}}\right)\right\}
\end{array}\right. \\
& \left\{\begin{array}{l}
\widetilde{P}_{n}=\left(\left|J_{\widetilde{x}}\right| T_{0}\right)^{-1}\left(\frac{\partial \widetilde{x}_{2}}{\partial x_{2}} P_{n}-\frac{\partial \widetilde{x}_{2}}{\partial x_{1}} Q_{n}\right) T_{0}, \\
\widetilde{Q}_{n}=\left(\left|J_{\widetilde{x}}\right| T_{0}\right)^{-1}\left(\frac{\partial \widetilde{x}_{1}}{\partial x_{1}} Q_{n}-\frac{\partial \widetilde{x}_{1}}{\partial x_{2}} P_{n}\right) T_{0}, \quad(n \geq 2) .
\end{array}\right.
\end{aligned}
$$

Then there exists a positive constant $\rho>0$ such that all the required properties in Proposition 4.1 except for the estimate (4.4) are satisfied.

We finally prove estimate (4.4). Since $\left(\partial \widetilde{x}_{i} / \partial x_{j}\right)(x)(i, j=1,2), T_{0}(x)$, $\left(\left|J_{\widetilde{x}}\right|(x) T_{0}(x)\right)^{-1}, \widetilde{P}_{i}(x)(i=0,1)$ and $\widetilde{Q}_{i}(x)(i=0,1)$ are holomorphic in $D_{\rho}$, we have

$$
\left|\frac{\partial \widetilde{x}_{i}}{\partial x_{j}}\right|_{\rho, \rho},\left\|T_{0}\right\|_{\rho, \rho},\left\|\left(\left|J_{\widetilde{x}}\right| T_{0}\right)^{-1}\right\|_{\rho, \rho},\left\|\widetilde{P}_{i}\right\|_{\rho, \rho},\left\|\widetilde{Q}_{i}\right\|_{\rho, \rho} \leq C^{\dagger}
$$

with some positive constant $C^{\dagger}$. Here we define $|f|_{\rho_{1}, \rho_{2}}$ for a holomorphic function $f(x)$ in $D_{\rho_{0}}\left(0<\rho_{1}, \rho_{2}<\rho_{0}\right)$ as follows:

$$
|f|_{\rho_{1}, \rho_{2}}=\sup _{\left|x_{1}\right| \leq \rho_{1},\left|x_{2}\right| \leq \rho_{2}}|f(x)| .
$$

Then, if we define positive constants $\widetilde{C}$ and $\widetilde{\alpha}$ by

$$
\widetilde{C}=\max \left\{C^{\dagger}, 2\left(C^{\dagger}\right)^{3} C\right\}, \quad \widetilde{\alpha}=\max \{1, \alpha\},
$$

the estimate (4.4) does really hold. In fact,

$$
\left\|\widetilde{P}_{0}\right\|_{\rho, \rho},\left\|\widetilde{Q}_{0}\right\|_{\rho, \rho} \leq C^{\dagger} \leq \widetilde{C}, \quad\left\|\widetilde{P}_{1}\right\|_{\rho, \rho},\left\|\widetilde{Q}_{1}\right\|_{\rho, \rho} \leq C^{\dagger} \leq \widetilde{C} \widetilde{\alpha}
$$

and it follows from (4.34) that

$$
\left\|\widetilde{P}_{n}\right\|_{\rho, \rho},\left\|\widetilde{Q}_{n}\right\|_{\rho, \rho} \leq 2\left(C^{\dagger}\right)^{3} C \alpha^{n} n ! \leq \widetilde{C} \widetilde{\alpha}^{n} n ! \quad \text { for } n \geq 2 .
$$

Finally, we prove Lemma 2.12 and Proposition 2.13 by using the construction of $\widetilde{x}(x)$.

Proof of Lemma 2.12. For simplicity, $\xi_{1, i}(x), \xi_{2, i}(x), \widetilde{\xi}_{1, i}(\widetilde{x})$ and $\widetilde{\xi}_{2, i}(\widetilde{x})$ are denoted by $\xi_{1}(x), \xi_{2}(x), \widetilde{\xi}_{1}(\widetilde{x})$ and $\widetilde{\xi}_{2}(\widetilde{x})$, respectively. Combining $(2.49),(2.50)$ and $(2.51)$ with (4.8), (4.12) and (4.16), we have

$$
\begin{aligned}
\xi_{1}(x)^{3}+a_{2}(x) \xi_{1}(x) & +a_{3}(x) \\
= & \left\{\widetilde{\xi}_{1}(\widetilde{x}(x)) \frac{\partial \widetilde{x}_{1}}{\partial x_{1}}+\left(\widetilde{\xi}_{1}(\widetilde{x}(x))\right)^{2} \frac{\partial \widetilde{x}_{2}}{\partial x_{1}}+\frac{\widetilde{x}_{2}}{3} \frac{\partial \widetilde{x}_{2}}{\partial x_{1}}\right\}^{3} \\
& +a_{2}(x)\left\{\widetilde{\xi}_{1}(\widetilde{x}(x)) \frac{\partial \widetilde{x}_{1}}{\partial x_{1}}+\left(\widetilde{\xi}_{1}(\widetilde{x}(x))\right)^{2} \frac{\partial \widetilde{x}_{2}}{\partial x_{1}}+\frac{\widetilde{x}_{2}}{3} \frac{\partial \widetilde{x}_{2}}{\partial x_{1}}\right\}+a_{3}(x)
\end{aligned}
$$




$$
\begin{aligned}
& =\left\{\frac{\widetilde{x}_{2}^{2}}{12}\left(\frac{\partial \widetilde{x}_{2}}{\partial x_{1}}\right)^{3}-\frac{3}{4} \widetilde{x}_{1} \frac{\partial \widetilde{x}_{1}}{\partial x_{1}}\left(\frac{\partial \widetilde{x}_{2}}{\partial x_{1}}\right)^{2}-\frac{\widetilde{x}_{2}}{2}\left(\frac{\partial \widetilde{x}_{1}}{\partial x_{1}}\right)^{2} \frac{\partial \widetilde{x}_{2}}{\partial x_{1}}+a_{2}(x) \frac{\partial \widetilde{x}_{2}}{\partial x_{1}}\right\}\left(\widetilde{\xi}_{1}(\widetilde{x}(x))\right)^{2} \\
& +\left\{\frac{\widetilde{x}_{2}^{2}}{12} \frac{\partial \widetilde{x}_{1}}{\partial x_{1}}\left(\frac{\partial \widetilde{x}_{2}}{\partial x_{1}}\right)^{2}-\frac{3}{4} \widetilde{x}_{1}\left(\frac{\partial \widetilde{x}_{1}}{\partial x_{1}}\right)^{2} \frac{\partial \widetilde{x}_{2}}{\partial x_{1}}-\frac{\widetilde{x}_{2}}{2}\left(\frac{\partial \widetilde{x}_{1}}{\partial x_{1}}\right)^{3}+a_{2}(x) \frac{\partial \widetilde{x}_{1}}{\partial x_{1}}\right\} \widetilde{\xi}_{1}(\widetilde{x}(x)) \\
& +\left\{\left(\frac{\widetilde{x}_{1}^{2}}{16}+\frac{\widetilde{x}_{2}^{3}}{27}\right)\left(\frac{\partial \widetilde{x}_{2}}{\partial x_{1}}\right)^{3}-\frac{\widetilde{x}_{1}}{4}\left(\frac{\partial \widetilde{x}_{1}}{\partial x_{1}}\right)^{3}-\frac{\widetilde{x}_{1} \widetilde{x}_{2}}{8} \frac{\partial \widetilde{x}_{1}}{\partial x_{1}}\left(\frac{\partial \widetilde{x}_{2}}{\partial x_{1}}\right)^{2}\right. \\
& \left.+\frac{a_{2}(x)}{3} \widetilde{x}_{2} \frac{\partial \widetilde{x}_{2}}{\partial x_{1}}+a_{3}(x)\right\}=0, \\
& b_{2}(x) \xi_{1}(x)^{2}+b_{1}(x) \xi_{1}(x)+b_{0}(x) \\
& =b_{2}(x)\left\{\widetilde{\xi}_{1}(\widetilde{x}(x)) \frac{\partial \widetilde{x}_{1}}{\partial x_{1}}+\left(\widetilde{\xi}_{1}(\widetilde{x}(x))\right)^{2} \frac{\partial \widetilde{x}_{2}}{\partial x_{1}}+\frac{\widetilde{x}_{2}}{3} \frac{\partial \widetilde{x}_{2}}{\partial x_{1}}\right\}^{2} \\
& +b_{1}(x)\left\{\widetilde{\xi}_{1}(\widetilde{x}(x)) \frac{\partial \widetilde{x}_{1}}{\partial x_{1}}+\left(\widetilde{\xi}_{1}(\widetilde{x}(x))\right)^{2} \frac{\partial \widetilde{x}_{2}}{\partial x_{1}}+\frac{\widetilde{x}_{2}}{3} \frac{\partial \widetilde{x}_{2}}{\partial x_{1}}\right\}+b_{0}(x) \\
& =\left[b_{2}(x)\left\{\left(\frac{\partial \widetilde{x}_{1}}{\partial x_{1}}\right)^{2}+\frac{\widetilde{x}_{2}}{6}\left(\frac{\partial \widetilde{x}_{2}}{\partial x_{1}}\right)^{2}\right\}+b_{1}(x) \frac{\partial \widetilde{x}_{2}}{\partial x_{1}}\right]\left(\widetilde{\xi}_{1}(\widetilde{x}(x))\right)^{2} \\
& +\left[b_{2}(x)\left\{-\frac{\widetilde{x}_{2}}{3} \frac{\partial \widetilde{x}_{1}}{\partial x_{1}} \frac{\partial \widetilde{x}_{2}}{\partial x_{1}}-\frac{\widetilde{x}_{1}}{4}\left(\frac{\partial \widetilde{x}_{2}}{\partial x_{1}}\right)^{2}\right\}+b_{1}(x) \frac{\partial \widetilde{x}_{1}}{\partial x_{1}}\right] \widetilde{\xi}_{1}(\widetilde{x}(x)) \\
& +\left[b_{2}(x)\left\{\frac{\widetilde{x}_{2}^{2}}{9}\left(\frac{\partial \widetilde{x}_{2}}{\partial x_{1}}\right)^{2}-\frac{\widetilde{x}_{1}}{2} \frac{\partial \widetilde{x}_{1}}{\partial x_{1}} \frac{\partial \widetilde{x}_{2}}{\partial x_{1}}\right\}+\frac{b_{1}(x)}{3} \widetilde{x}_{2} \frac{\partial \widetilde{x}_{2}}{\partial x_{1}}+b_{0}(x)\right] \\
& =\frac{\partial \widetilde{x}_{2}}{\partial x_{2}}\left(\widetilde{\xi}_{1}(\widetilde{x})\right)^{2}+\frac{\partial \widetilde{x}_{1}}{\partial x_{2}} \widetilde{\xi}_{1}(\widetilde{x}(x))+\frac{\widetilde{x}_{2}}{3} \frac{\partial \widetilde{x}_{2}}{\partial x_{2}} \\
& =\frac{\partial \widetilde{x}_{1}}{\partial x_{2}} \widetilde{\xi}_{1}(\widetilde{x}(x))+\frac{\partial \widetilde{x}_{2}}{\partial x_{2}} \widetilde{\xi}_{2}(\widetilde{x}(x))=\xi_{2}(x) .
\end{aligned}
$$

Proof of Proposition 2.13. Using (2.51), we have

$$
\begin{aligned}
\left(\xi_{1, i}(x)-\right. & \left.\xi_{1, i^{\prime}}(x), \xi_{2, i}(x)-\xi_{2, i^{\prime}}(x)\right) \\
& =\left(\widetilde{\xi}_{1, i}(\widetilde{x}(x))-\widetilde{\xi}_{1, i^{\prime}}(\widetilde{x}(x)), \widetilde{\xi}_{2, i}(\widetilde{x}(x))-\widetilde{\xi}_{2, i^{\prime}}(\widetilde{x}(x))\right)\left(\begin{array}{ll}
\frac{\partial \widetilde{x}_{1}}{\partial x_{1}}(x) & \frac{\partial \widetilde{x}_{1}}{\partial x_{2}}(x) \\
\frac{\partial \widetilde{x}_{2}}{\partial x_{1}}(x) & \frac{\partial \widetilde{x}_{2}}{\partial x_{2}}(x)
\end{array}\right) .
\end{aligned}
$$

Hence, by the definition of turning points and Lemma 4.7, we immediately obtain Proposition 2.13.

\section{§4.2. Construction of the transformation, II}

As a consequence of the argument of the previous subsection, we may assume that the completely integrable system in question has the form 


$$
\begin{cases}\eta^{-1} \frac{\partial}{\partial x_{1}} \Psi=P(x, \eta) \Psi, & P(x, \eta)=\sum_{n \geq 0} \eta^{-n} P_{n}(x), \\ \eta^{-1} \frac{\partial}{\partial x_{2}} \Psi=Q(x, \eta) \Psi, & Q(x, \eta)=\sum_{n \geq 0} \eta^{-n} Q_{n}(x) .\end{cases}
$$

Here

$$
P_{0}=\left(\begin{array}{ccc}
0 & 1 & 0 \\
0 & 0 & 1 \\
-x_{1} / 4 & -x_{2} / 2 & 0
\end{array}\right), \quad Q_{0}=P_{0}^{2}+\frac{x_{2}}{3}
$$

and $P_{n}(x)$ and $Q_{n}(x)$ are $3 \times 3$ matrices with holomorphic entries in $D_{\rho_{0}}$ satisfying

$$
\left\|P_{n}\right\|_{\rho_{0}, \rho_{0}},\left\|Q_{n}\right\|_{\rho_{0}, \rho_{0}} \leq C \alpha^{n} n ! \quad(n \geq 0)
$$

with some positive constants $C$ and $\alpha$.

In this subsection, we prove

Proposition 4.10. There exist a sufficiently small positive constant $0<\rho<\rho_{0}$ and $3 \times 3$ matrices $T_{0}(x), T_{1}(x)$ with the following properties:

- Every entry of $T_{0}(x)$ and $T_{1}(x)$ is holomorphic in $D_{\rho}$ and $\operatorname{det} T_{0}(x) \neq 0\left(x \in D_{\rho}\right)$.

- The transformation

$$
\Psi(x, \eta)=T(x, \eta) \widetilde{\Psi}(x, \eta), \quad T(x, \eta)=T_{0}(x)+\eta^{-1} T_{1}(x),
$$

transforms (4.35) into

$$
\begin{cases}\eta^{-1} \frac{\partial}{\partial x_{1}} \widetilde{\Psi}=\widetilde{P}(x, \eta) \widetilde{\Psi}, & \widetilde{P}(x, \eta)=\sum_{n \geq 0} \eta^{-n} \widetilde{P}_{n}(x), \\ \eta^{-1} \frac{\partial}{\partial x_{2}} \widetilde{\Psi}=\widetilde{Q}(x, \eta) \widetilde{\Psi}, & \widetilde{Q}(x, \eta)=\sum_{n \geq 0} \eta^{-n} \widetilde{Q}_{n}(x),\end{cases}
$$

where

$$
\widetilde{P}_{0}=P_{0}, \quad \widetilde{P}_{1}=0, \quad \widetilde{Q}_{0}=P_{0}^{2}+\frac{x_{2}}{3}, \quad \widetilde{Q}_{1}=\frac{\partial P_{0}}{\partial x_{1}},
$$

and $\widetilde{P}_{n}(x)$ and $\widetilde{Q}_{n}(x)$ are $3 \times 3$ matrices with holomorphic entries in $D_{\rho}$ satisfying

$$
\left\|\widetilde{P}_{n}\right\|_{\rho, \rho},\left\|\widetilde{Q}_{n}\right\|_{\rho, \rho} \leq \widetilde{C} \widetilde{\alpha}^{n} n ! \quad(n \geq 0)
$$

with some positive constants $\widetilde{C}$ and $\widetilde{\alpha}$.

Since the system (4.37) is obtained from (4.35) through the transformation (4.36), $T(x, \eta)$ should satisfy the relation

$$
\left\{\begin{array}{l}
T \widetilde{P}+\eta^{-1} \frac{\partial T}{\partial x_{1}}=P T \\
T \widetilde{Q}+\eta^{-1} \frac{\partial T}{\partial x_{2}}=Q T
\end{array}\right.
$$


that is,

$$
\begin{aligned}
& \left\{\begin{array}{l}
{\left[P_{0}, T_{0}\right]=0,} \\
{\left[Q_{0}, T_{0}\right]=0,}
\end{array}\right. \\
& \left\{\begin{array}{l}
{\left[P_{0}, T_{1}\right]=\frac{\partial T_{0}}{\partial x_{1}}-P_{1} T_{0},} \\
{\left[Q_{0}, T_{1}\right]=\frac{\partial T_{0}}{\partial x_{2}}+T_{0} \frac{\partial P_{0}}{\partial x_{1}}-Q_{1} T_{0},}
\end{array}\right. \\
& \left\{\begin{array}{l}
\frac{\partial T_{1}}{\partial x_{1}}+T_{0} \widetilde{P}_{2}-P_{2} T_{0}-P_{1} T_{1}=0, \\
\frac{\partial T_{1}}{\partial x_{2}}+T_{0} \widetilde{Q}_{2}-Q_{2} T_{0}+T_{1} \frac{\partial P_{0}}{\partial x_{1}}-Q_{1} T_{1}=0,
\end{array}\right. \\
& \left\{\begin{array}{l}
T_{0} \widetilde{P}_{n}-P_{n} T_{0}+T_{1} \widetilde{P}_{n-1}-P_{n-1} T_{1}=0, \\
T_{0} \widetilde{Q}_{n}-Q_{n} T_{0}+T_{1} \widetilde{Q}_{n-1}-Q_{n-1} T_{1}=0,
\end{array}\right.
\end{aligned}
$$

First, by $\left[T_{0}, P_{0}\right]=0$ and the same argument as in the proof of Lemma 2.4, we find that

$$
T_{0}=c_{0,2}(x) P_{0}^{2}+c_{0,1}(x) P_{0}+c_{0,0}(x)
$$

with some holomorphic functions $c_{0, k}(x)(k=0,1,2)$ near $x=0$. Note that $T_{0}(x)$ then automatically satisfies $\left[T_{0}, Q_{0}\right]=0$ since $\left[P_{0}, Q_{0}\right]=0$.

We next consider

$$
\left\{\begin{array}{l}
{\left[P_{0}, T_{1}\right]=\frac{\partial T_{0}}{\partial x_{1}}-P_{1} T_{0}=: F_{1}} \\
{\left[Q_{0}, T_{1}\right]=\frac{\partial T_{0}}{\partial x_{2}}+T_{0} \frac{\partial P_{0}}{\partial x_{1}}-Q_{1} T_{0}=: F_{2} .}
\end{array}\right.
$$

For this system, we have

Lemma 4.11. If $F_{1}$ and $F_{2}$ satisfy

$$
\left\{\begin{array}{l}
\operatorname{tr}\left(F_{1} P_{0}^{k}\right)=0 \quad(k=0,1,2), \\
F_{2}=P_{0} F_{1}+F_{1} P_{0},
\end{array}\right.
$$

then the system (4.45) has a solution $T_{1}$.

Proof. Let $T_{1}(x)$ be a $3 \times 3$ matrix with holomorphic entries near $x=0$ defined by

$$
T_{1}=\Lambda F_{1}+\Lambda^{2} F_{1} P_{0}, \quad \Lambda=\left(\begin{array}{ccc}
0 & 0 & 0 \\
1 & 0 & 0 \\
0 & 1 & 0
\end{array}\right)
$$


Then, by the explicit calculation of matrix entries, we find

$$
\left[P_{0}, T_{1}\right]=F_{1}-\left(\begin{array}{ccc}
0 & 0 & 0 \\
0 & 0 & 0 \\
\operatorname{tr}\left(F_{1} P_{0}^{2}\right)+\frac{x_{2}}{2} \operatorname{tr}\left(F_{1}\right) & \operatorname{tr}\left(F_{1} P_{0}\right) & \operatorname{tr}\left(F_{1}\right)
\end{array}\right) .
$$

Hence, using $\operatorname{tr}\left(F_{1} P_{0}^{k}\right)=0(k=0,1,2)$, we find that $T_{1}(x)$ satisfies the first equation of (4.45). Furthermore, using $F_{2}=P_{0} F_{1}+F_{1} P_{0}$, we obtain

$\left[Q_{0}, T_{1}\right]=\left[P_{0}^{2}, T_{1}\right]=P_{0}^{2} T_{1}-T_{1} P_{0}^{2}=P_{0}\left[P_{0}, T_{1}\right]+\left[P_{0}, T_{1}\right] P_{0}=P_{0} F_{1}+F_{1} P_{0}=F_{2}$.

In what follows, we try to determine the matrix $T_{0}$ of the form (4.44) so that the relations (4.46) are satisfied.

Sublemma 4.12. There exist holomorphic functions $d_{k}(x)(k=0,1,2)$ near $x=0$ which satisfy

$$
Q_{1}-\frac{\partial P_{0}}{\partial x_{1}}-P_{0} P_{1}-P_{1} P_{0}=d_{2}(x) P_{0}^{2}+d_{1}(x) P_{0}+d_{0}(x) .
$$

Proof. Using the compatibility condition for the system (4.35) and the explicit form of $P_{0}$ and $Q_{0}$, we have

$$
\begin{aligned}
{\left[P_{0}, Q_{1}-\frac{\partial P_{0}}{\partial x_{1}}-\left(P_{0} P_{1}+P_{1} P_{0}\right)\right] } & =\left[P_{0}, Q_{1}-\frac{\partial P_{0}}{\partial x_{1}}\right]-\left[P_{0}, P_{0} P_{1}+P_{1} P_{0}\right] \\
& =\left[P_{0}, Q_{1}\right]+\frac{\partial P_{0}}{\partial x_{2}}-\frac{\partial Q_{0}}{\partial x_{1}}-\left[Q_{0}, P_{1}\right]=0 .
\end{aligned}
$$

Then, by the same argument as in the proof of Lemma 2.4, we get (4.47).

Using this sublemma, we find

Lemma 4.13. The relation (4.46) implies that $\left(c_{0,0}(x), c_{0,1}(x), c_{0,2}(x)\right)$ satisfies the system of partial differential equations

$$
\left\{\begin{array}{c}
\left(3 x_{1}+4 x_{2} P_{0}^{T}\right) \frac{\partial}{\partial x_{1}}\left(\begin{array}{c}
c_{0,0} \\
c_{0,1} \\
c_{0,2}
\end{array}\right) \\
=\left\{\operatorname{diag}(0,-1,-2)+x_{1} e_{0}(x)-4 e_{2}(x) P_{0}-4 e_{1}(x) P_{0}^{2}\right\}^{T}\left(\begin{array}{l}
c_{0,0} \\
c_{0,1} \\
c_{0,2}
\end{array}\right), \\
\frac{\partial}{\partial x_{2}}\left(\begin{array}{l}
c_{0,0} \\
c_{0,1} \\
c_{0,2}
\end{array}\right)=2 P_{0}^{T} \frac{\partial}{\partial x_{1}}\left(\begin{array}{l}
c_{0,0} \\
c_{0,1} \\
c_{0,2}
\end{array}\right)+\left(d_{2}(x) P_{0}^{2}+d_{1}(x) P_{0}+d_{0}(x)\right)^{T}\left(\begin{array}{l}
c_{0,0} \\
c_{0,1} \\
c_{0,2}
\end{array}\right),
\end{array}\right.
$$


where $A^{T}$ designates the transposed matrix of $A, \operatorname{diag}\left(a_{1}, a_{2}, a_{3}\right)$ is a diagonal matrix whose $(i, i)$ entry is $a_{i}$, and $e_{k}(x)=\operatorname{tr}\left(P_{1} P_{0}^{k}\right)(k=0,1,2)$. Conversely, if $\left(c_{0,0}(x), c_{0,1}(x), c_{0,2}(x)\right)$ is a holomorphic solution of (4.48), then (4.46) holds.

Proof. First, we consider

$$
\operatorname{tr}\left(F_{1} P_{0}^{k}\right)=0 \quad(k=0,1,2) .
$$

By the explicit form of $P_{0}$, we have

$$
\begin{aligned}
& \operatorname{tr}\left(P_{0}\right)=0, \quad \operatorname{tr}\left(P_{0}^{2}\right)=-x_{2}, \quad \operatorname{tr}\left(\frac{\partial P_{0}}{\partial x_{1}}\right)=0, \\
& \operatorname{tr}\left(P_{0} \frac{\partial P_{0}}{\partial x_{1}}\right)=0, \quad \operatorname{tr}\left(P_{0}^{2} \frac{\partial P_{0}}{\partial x_{1}}\right)=-\frac{1}{4} .
\end{aligned}
$$

It follows from these relations that

$$
\begin{aligned}
\operatorname{tr}\left(F_{1}\right)= & -x_{2} \frac{\partial c_{0,2}}{\partial x_{1}}+3 \frac{\partial c_{0,0}}{\partial x_{1}}-e_{2}(x) c_{0,2}-e_{1}(x) c_{0,1}-e_{0}(x) c_{0,0} \\
\operatorname{tr}\left(F_{1} P_{0}\right)= & -\frac{3}{4} x_{1} \frac{\partial c_{0,2}}{\partial x_{1}}-x_{2} \frac{\partial c_{0,1}}{\partial x_{1}}-\left(\frac{1}{2}-\frac{x_{2}}{2} e_{1}(x)-\frac{x_{1}}{4} e_{0}(x)\right) c_{0,2} \\
& -e_{2}(x) c_{0,1}-e_{1}(x) c_{0,0}, \\
\operatorname{tr}\left(F_{1} P_{0}^{2}\right)= & \frac{x_{2}^{2}}{2} \frac{\partial c_{0,2}}{\partial x_{1}}-\frac{3}{4} x_{1} \frac{\partial c_{0,1}}{\partial x_{1}}-x_{2} \frac{\partial c_{0,0}}{\partial x_{1}}-\left(-\frac{x_{2}}{2} e_{2}(x)-\frac{x_{1}}{4} e_{1}(x)\right) c_{0,2} \\
& -\left(\frac{1}{4}-\frac{x_{2}}{2} e_{1}(x)-\frac{x_{1}}{4} e_{0}(x)\right) c_{0,1}-e_{2}(x) c_{0,0}
\end{aligned}
$$

If we multiply (4.50) (resp., (4.51), (4.52)) by $x_{1}$ (resp., -4, -4), we get the first equation of (4.48).

Next, we consider

$$
F_{2}=P_{0} F_{1}+F_{1} P_{0}
$$

Using (4.47), we find that this relation is equivalent to

$$
\begin{aligned}
& \frac{\partial T_{0}}{\partial x_{2}}+\left[T_{0}, \frac{\partial P_{0}}{\partial x_{1}}\right]-P_{0} \frac{\partial T_{0}}{\partial x_{1}}-\frac{\partial T_{0}}{\partial x_{1}} P_{0} \\
& \quad=\left(F_{2}+Q_{1} T_{0}-\frac{\partial P_{0}}{\partial x_{1}} T_{0}\right)-P_{0}\left(F_{1}+P_{1} T_{0}\right)-\left(F_{1}+P_{1} T_{0}\right) P_{0} \\
& \quad=\left(Q_{1}-\frac{\partial P_{0}}{\partial x_{1}}-P_{0} P_{1}-P_{1} P_{0}\right) T_{0}=\left(d_{2}(x) P_{0}^{2}+d_{1}(x) P_{0}+d_{0}(x)\right) T_{0} .
\end{aligned}
$$

Note that

$$
\frac{\partial P_{0}}{\partial x_{2}}-2 \frac{\partial P_{0}}{\partial x_{1}} P_{0}=0
$$


by the explicit form of $P_{0}$. Hence, as

$$
P_{0}^{3}+\frac{x_{2}}{2} P_{0}+\frac{x_{1}}{4}=0
$$

the left-hand side of (4.54) becomes

$$
\begin{aligned}
& \text { (4.57) } \quad \frac{\partial T_{0}}{\partial x_{2}}+\left[T_{0}, \frac{\partial P_{0}}{\partial x_{1}}\right]-P_{0} \frac{\partial T_{0}}{\partial x_{1}}-\frac{\partial T_{0}}{\partial x_{1}} P_{0} \\
& =\left(\frac{\partial c_{0,2}}{\partial x_{2}}-2 \frac{\partial c_{0,1}}{\partial x_{1}}\right) P_{0}^{2}+\left(\frac{\partial c_{0,1}}{\partial x_{2}}+x_{2} \frac{\partial c_{0,2}}{\partial x_{1}}-2 \frac{\partial c_{0,0}}{\partial x_{1}}\right) P_{0}+\left(\frac{\partial c_{0,0}}{\partial x_{2}}+\frac{x_{1}}{2} \frac{\partial c_{0,2}}{\partial x_{1}}\right) \\
& \quad+c_{0,2}(x)\left\{\left(\frac{\partial P_{0}}{\partial x_{2}}-2 \frac{\partial P_{0}}{\partial x_{1}} P_{0}\right) P_{0}+P_{0}\left(\frac{\partial P_{0}}{\partial x_{2}}-2 \frac{\partial P_{0}}{\partial x_{1}} P_{0}\right)\right\} \\
& \quad+c_{0,1}(x)\left(\frac{\partial P_{0}}{\partial x_{2}}-2 \frac{\partial P_{0}}{\partial x_{1}} P_{0}\right) \\
& =\left(\frac{\partial c_{0,2}}{\partial x_{2}}-2 \frac{\partial c_{0,1}}{\partial x_{1}}\right) P_{0}^{2}+\left(\frac{\partial c_{0,1}}{\partial x_{2}}+x_{2} \frac{\partial c_{0,2}}{\partial x_{1}}-2 \frac{\partial c_{0,0}}{\partial x_{1}}\right) P_{0}+\left(\frac{\partial c_{0,0}}{\partial x_{2}}+\frac{x_{1}}{2} \frac{\partial c_{0,2}}{\partial x_{1}}\right) .
\end{aligned}
$$

On the other hand, in view of (4.56), we can rewrite the right-hand side of (4.54) as

$$
\begin{aligned}
& \left(d_{2}(x) P_{0}^{2}+d_{1}(x) P_{0}+d_{0}(x)\right) T_{0} \\
= & \left\{\left(d_{0}(x)-\frac{x_{2}}{2} d_{2}(x)\right) c_{0,2}+d_{1}(x) c_{0,1}+d_{2}(x) c_{0,0}\right\} P_{0}^{2} \\
+ & \left\{\left(-\frac{x_{2}}{2} d_{1}(x)-\frac{x_{1}}{4} d_{2}(x)\right) c_{0,2}+\left(d_{0}(x)-\frac{x_{2}}{2} d_{2}(x)\right) c_{0,1}+d_{1}(x) c_{0,0}\right\} P_{0} \\
+ & \left\{\left(-\frac{x_{1}}{4} d_{1}(x)\right) c_{0,2}+\left(-\frac{x_{1}}{4} d_{2}(x)\right) c_{0,1}+d_{0}(x) c_{0,0}\right\} .
\end{aligned}
$$

Comparing (4.57) and (4.58), we obtain

$$
\begin{aligned}
\frac{\partial c_{0,2}}{\partial x_{2}}-2 \frac{\partial c_{0,1}}{\partial x_{1}}= & \left(d_{0}(x)-\frac{x_{2}}{2} d_{2}(x)\right) c_{0,2}+d_{1}(x) c_{0,1}+d_{2}(x) c_{0,0}, \\
\frac{\partial c_{0,1}}{\partial x_{2}}+x_{2} \frac{\partial c_{0,2}}{\partial x_{1}}-2 \frac{\partial c_{0,0}}{\partial x_{1}}= & \left(-\frac{x_{2}}{2} d_{1}(x)-\frac{x_{1}}{4} d_{2}(x)\right) c_{0,2}+\left(d_{0}(x)-\frac{x_{2}}{2} d_{2}(x)\right) c_{0,1} \\
& +d_{1}(x) c_{0,0}, \\
\frac{\partial c_{0,0}}{\partial x_{2}}+\frac{x_{1}}{2} \frac{\partial c_{0,2}}{\partial x_{1}}= & \left(-\frac{x_{1}}{4} d_{1}(x)\right) c_{0,2}+\left(-\frac{x_{1}}{4} d_{2}(x)\right) c_{0,1}+d_{0}(x) c_{0,0} .
\end{aligned}
$$

Thus we also get the second equation of (4.48).

The "converse" part is clear from the above argument.

We now prove the existence of a holomorphic solution of the system (4.48). 
Lemma 4.14. Let

$$
\begin{aligned}
& 3 x_{1} \frac{d}{d x_{1}}\left(\begin{array}{c}
c_{0,0} \\
c_{0,1} \\
c_{0,2}
\end{array}\right) \\
& =\left.\left\{\operatorname{diag}(0,-1,-2)+x_{1} e_{0}(x)-4 e_{2}(x) P_{0}-4 e_{1}(x) P_{0}^{2}\right\}^{T}\right|_{x_{2}=0}\left(\begin{array}{c}
c_{0,0} \\
c_{0,1} \\
c_{0,2}
\end{array}\right)
\end{aligned}
$$

be a system of ordinary differential equations obtained by restricting the first equation of (4.48) to $x_{2}=0$. Then (4.59) has a holomorphic solution $\left(c_{0,0}\left(x_{1}, 0\right), c_{0,1}\left(x_{1}, 0\right), c_{0,2}\left(x_{1}, 0\right)\right)$ near $x_{1}=0$ satisfying

$$
\left(\begin{array}{c}
c_{0,0}(0) \\
c_{0,1}(0) \\
c_{0,2}(0)
\end{array}\right)=\left(\begin{array}{c}
1 \\
-4 e_{2}(0) \\
-2 e_{1}(0)+8 e_{2}(0)^{2}
\end{array}\right) .
$$

In the proof of this lemma we use the following

Theorem 4.15 ([W1]). Let $A(t)$ be an $n \times n$ matrix with holomorphic entries near $t=0$. Suppose that $A(0)$ has no eigenvalues that differ from each other by positive integers. Then there exists an $n \times n$ matrix $B(t)$ with holomorphic entries near $t=0$ with $B(0)=I$, such that the transformation

$$
W=B(t) \widetilde{W}
$$

reduces the system of ordinary differential equations

$$
t \frac{d W}{d t}=A(t) W
$$

to the system

$$
t \frac{d \widetilde{W}}{d t}=A(0) \widetilde{W}
$$

Proof of Lemma 4.14. By the explicit form of $P_{0}$, the eigenvalues of $\left.(1 / 3)\left\{\operatorname{diag}(0,-1,-2)+x_{1} e_{0}(x)-4 e_{2}(x) P_{0}-4 e_{1}(x) P_{0}^{2}\right\}^{T}\right|_{x=0}$ are given by $0,-1 / 3$ and $-2 / 3$. Hence, there exists a $3 \times 3$ matrix $B\left(x_{1}\right)$ which has the properties of Theorem 4.15. The transformation

$$
\left(\begin{array}{l}
c_{0,0} \\
c_{0,1} \\
c_{0,2}
\end{array}\right)=B\left(x_{1}\right) \widetilde{\left(\begin{array}{l}
c_{0,0} \\
c_{0,1} \\
c_{0,2}
\end{array}\right)}
$$


transforms the system (4.59) into

$$
x_{1} \frac{d}{d x_{1}} \widetilde{\left(\begin{array}{l}
c_{0,0} \\
c_{0,1} \\
c_{0,2}
\end{array}\right)}=\frac{1}{3}\left(\begin{array}{ccc}
0 & 0 & 0 \\
-4 e_{2}(0) & -1 & 0 \\
-4 e_{1}(0) & -4 e_{2}(0) & -2
\end{array}\right) \widetilde{\left(\begin{array}{l}
c_{0,0} \\
c_{0,1} \\
c_{0,2}
\end{array}\right)} .
$$

Since

$$
\widetilde{\left(\begin{array}{c}
c_{0,0} \\
c_{0,1} \\
c_{0,2}
\end{array}\right)}=\left(\begin{array}{c}
1 \\
-4 e_{2}(0) \\
-2 e_{1}(0)+8 e_{2}(0)^{2}
\end{array}\right)
$$

is a solution of (4.64), it follows that

$$
\left(\begin{array}{l}
c_{0,0} \\
c_{0,1} \\
c_{0,2}
\end{array}\right)=B\left(x_{1}\right)\left(\begin{array}{c}
1 \\
-4 e_{2}(0) \\
-2 e_{1}(0)+8 e_{2}(0)^{2}
\end{array}\right)
$$

is a solution of (4.59). Since $B(0)=I,(4.65)$ also satisfies (4.60).

Lemma 4.16. Let $\left(c_{0,0}\left(x_{1}, 0\right), c_{0,1}\left(x_{1}, 0\right), c_{0,2}\left(x_{1}, 0\right)\right)$ be a holomorphic solution near $x_{1}=0$ given in Lemma 4.14. Then the initial value problem

$$
\left\{\begin{array}{l}
\frac{\partial}{\partial x_{2}}\left(\begin{array}{l}
c_{0,0} \\
c_{0,1} \\
c_{0,2}
\end{array}\right)=2 P_{0}^{T} \frac{\partial}{\partial x_{1}}\left(\begin{array}{l}
c_{0,0} \\
c_{0,1} \\
c_{0,2}
\end{array}\right)+\left(d_{2}(x) P_{0}^{2}+d_{1}(x) P_{0}+d_{0}(x)\right)^{T}\left(\begin{array}{l}
c_{0,0} \\
c_{0,1} \\
c_{0,2}
\end{array}\right) \\
\left.\left(\begin{array}{l}
c_{0,0} \\
c_{0,1} \\
c_{0,2}
\end{array}\right)\right|_{x_{2}=0}=\left(\begin{array}{l}
c_{0,0}\left(x_{1}, 0\right) \\
c_{0,1}\left(x_{1}, 0\right) \\
c_{0,2}\left(x_{1}, 0\right)
\end{array}\right)
\end{array}\right.
$$

has a holomorphic solution near $x=0$.

This lemma is an immediate consequence of the Cauchy-Kowalevski theorem. Furthermore, we can verify

Lemma 4.17. The holomorphic solution $\left(c_{0,0}(x), c_{0,1}(x), c_{0,2}(x)\right)$ given by Lemma 4.16 satisfies the first equation of (4.48).

Proof. By the argument of Lemma 4.13, it suffices to prove that

$$
T_{0}=c_{0,2}(x) P_{0}^{2}+c_{0,1}(x) P_{0}+c_{0,0}(x)
$$

satisfies

$$
\operatorname{tr}\left\{\left(\frac{\partial T_{0}}{\partial x_{1}}-P_{1} T_{0}\right) P_{0}^{k}\right\}=0 \quad(k=0,1,2)
$$


Let $f_{0, k}(x)(k=0,1,2)$ be holomorphic functions defined by

$$
f_{0, k}=\operatorname{tr}\left\{\left(\frac{\partial T_{0}}{\partial x_{1}}-P_{1} T_{0}\right) P_{0}^{k}\right\} \quad(k=0,1,2) .
$$

We will prove that $\left(f_{0,0}(x), f_{0,1}(x), f_{0,2}(x)\right)$ satisfies the system of partial differential equations

$$
\frac{\partial}{\partial x_{2}}\left(\begin{array}{l}
f_{0,0} \\
f_{0,1} \\
f_{0,2}
\end{array}\right)=2 \frac{\partial}{\partial x_{1}}\left\{P_{0}\left(\begin{array}{l}
f_{0,0} \\
f_{0,1} \\
f_{0,2}
\end{array}\right)\right\}+\left(d_{2}(x) P_{0}^{2}+d_{1}(x) P_{0}+d_{0}(x)\right)\left(\begin{array}{l}
f_{0,0} \\
f_{0,1} \\
f_{0,2}
\end{array}\right) .
$$

For $k=0,1,2$, we have

$$
\begin{aligned}
& \operatorname{tr}\left(\left[P_{2}, Q_{0}\right] T_{0} P_{0}^{k}\right)=\operatorname{tr}\left(P_{2} Q_{0} T_{0} P_{0}^{k}-Q_{0} P_{2} T_{0} P_{0}^{k}\right)=\operatorname{tr}\left[P_{2} T_{0} P_{0}^{k}, Q_{0}\right]=0, \\
& \operatorname{tr}\left(\left[P_{0}, Q_{2}\right] T_{0} P_{0}^{k}\right)=\operatorname{tr}\left(P_{0} Q_{2} T_{0} P_{0}^{k}-Q_{2} P_{0} T_{0} P_{0}^{k}\right)=\operatorname{tr}\left[P_{0}, Q_{2} T_{0} P_{0}^{k}\right]=0
\end{aligned}
$$

in view of $\left[P_{0}, Q_{0}\right]=0$ and (4.44). Furthermore, it follows from (4.47) that

$$
\begin{aligned}
\operatorname{tr} & \left(\left[P_{1}, Q_{1}\right] T_{0} P_{0}^{k}\right) \\
= & \operatorname{tr}\left(\left[P_{1}, \frac{\partial P_{0}}{\partial x_{1}}+P_{0} P_{1}+P_{1} P_{0}+d_{2}(x) P_{0}^{2}+d_{1}(x) P_{0}+d_{0}(x)\right] T_{0} P_{0}^{k}\right) \\
= & \operatorname{tr}\left(\left[P_{1}, \frac{\partial P_{0}}{\partial x_{1}}+d_{2}(x) P_{0}^{2}+d_{1}(x) P_{0}+d_{0}(x)\right] T_{0} P_{0}^{k}\right) \\
& +\operatorname{tr}\left\{\left(P_{1} P_{0} P_{1}+P_{1}^{2} P_{0}-P_{0} P_{1}^{2}-P_{1} P_{0} P_{1}\right) T_{0} P_{0}^{k}\right\} \\
= & \operatorname{tr}\left(\left[P_{1}, \frac{\partial P_{0}}{\partial x_{1}}+d_{2}(x) P_{0}^{2}+d_{1}(x) P_{0}+d_{0}(x)\right] T_{0} P_{0}^{k}\right)+\operatorname{tr}\left[P_{1}^{2} T_{0} P_{0}^{k}, P_{0}\right] \\
= & \operatorname{tr}\left(\left[P_{1}, \frac{\partial P_{0}}{\partial x_{1}}+d_{2}(x) P_{0}^{2}+d_{1}(x) P_{0}+d_{0}(x)\right] T_{0} P_{0}^{k}\right) .
\end{aligned}
$$

By using these relations, the compatibility conditions, (4.47), (4.54) and (4.55), we obtain

$$
\begin{aligned}
\frac{\partial f_{0,0}}{\partial x_{2}}= & \operatorname{tr}\left(\frac{\partial^{2} T_{0}}{\partial x_{1} \partial x_{2}}-\frac{\partial P_{1}}{\partial x_{2}} T_{0}-P_{1} \frac{\partial T_{0}}{\partial x_{2}}\right) \\
= & \operatorname{tr}\left[\frac{\partial}{\partial x_{1}}\left\{-\left[T_{0}, \frac{\partial P_{0}}{\partial x_{1}}\right]+P_{0} \frac{\partial T_{0}}{\partial x_{1}}+\frac{\partial T_{0}}{\partial x_{1}} P_{0}+\left(Q_{1}-\frac{\partial P_{0}}{\partial x_{1}}-P_{0} P_{1}-P_{1} P_{0}\right) T_{0}\right\}\right. \\
& +\left\{\left[P_{2}, Q_{0}\right]+\left[P_{1}, Q_{1}\right]+\left[P_{0}, Q_{2}\right]-\frac{\partial Q_{1}}{\partial x_{1}}\right\} T_{0} \\
& \left.-P_{1}\left\{-\left[T_{0}, \frac{\partial P_{0}}{\partial x_{1}}\right]+P_{0} \frac{\partial T_{0}}{\partial x_{1}}+\frac{\partial T_{0}}{\partial x_{1}} P_{0}+\left(Q_{1}-\frac{\partial P_{0}}{\partial x_{1}}-P_{0} P_{1}-P_{1} P_{0}\right) T_{0}\right\}\right]
\end{aligned}
$$




$$
\begin{aligned}
& =2 \frac{\partial}{\partial x_{1}} \operatorname{tr}\left\{\left(\frac{\partial T_{0}}{\partial x_{1}}-P_{1} T_{0}\right) P_{0}\right\}+\operatorname{tr}\left\{\left(\frac{\partial T_{0}}{\partial x_{1}}-P_{1} T_{0}\right)\left(d_{2}(x) P_{0}^{2}+d_{1}(x) P_{0}+d_{0}(x)\right)\right\} \\
& =2 \frac{\partial f_{0,1}}{\partial x_{1}}+d_{2}(x) f_{0,2}+d_{1}(x) f_{0,1}+d_{0}(x) f_{0,0}, \\
& \frac{\partial f_{0,1}}{\partial x_{2}}=\operatorname{tr}\left\{\left(\frac{\partial^{2} T_{0}}{\partial x_{1} \partial x_{2}}-\frac{\partial P_{1}}{\partial x_{2}} T_{0}-P_{1} \frac{\partial T_{0}}{\partial x_{2}}\right) P_{0}+\left(\frac{\partial T_{0}}{\partial x_{1}}-P_{1} T_{0}\right) \frac{\partial P_{0}}{\partial x_{2}}\right\} \\
& =\operatorname{tr}\left[\frac{\partial}{\partial x_{1}}\left\{-\left[T_{0}, \frac{\partial P_{0}}{\partial x_{1}}\right]+P_{0} \frac{\partial T_{0}}{\partial x_{1}}+\frac{\partial T_{0}}{\partial x_{1}} P_{0}+\left(Q_{1}-\frac{\partial P_{0}}{\partial x_{1}}-P_{0} P_{1}-P_{1} P_{0}\right) T_{0}\right\} P_{0} .\right. \\
& +\left\{\left[P_{2}, Q_{0}\right]+\left[P_{1}, Q_{1}\right]+\left[P_{0}, Q_{2}\right]-\frac{\partial Q_{1}}{\partial x_{1}}\right\} T_{0} P_{0} \\
& -P_{1}\left\{-\left[T_{0}, \frac{\partial P_{0}}{\partial x_{1}}\right]+P_{0} \frac{\partial T_{0}}{\partial x_{1}}+\frac{\partial T_{0}}{\partial x_{1}} P_{0}+\left(Q_{1}-\frac{\partial P_{0}}{\partial x_{1}}-P_{0} P_{1}-P_{1} P_{0}\right) T_{0}\right\} P_{0} \\
& \left.+\left(\frac{\partial T_{0}}{\partial x_{1}}-P_{1} T_{0}\right)\left(2 \frac{\partial P_{0}}{\partial x_{1}} P_{0}\right)\right] \\
& =2 \frac{\partial}{\partial x_{1}} \operatorname{tr}\left\{\left(\frac{\partial T_{0}}{\partial x_{1}}-P_{1} T_{0}\right) P_{0}^{2}\right\} \\
& +\operatorname{tr}\left\{\left(\frac{\partial T_{0}}{\partial x_{1}}-P_{1} T_{0}\right)\left(d_{2}(x) P_{0}^{3}+d_{1}(x) P_{0}^{2}+d_{0}(x) P_{0}\right)\right\} \\
& =2 \frac{\partial f_{0,2}}{\partial x_{1}}+d_{1}(x) f_{0,2}+\left(-\frac{x_{2}}{2} d_{2}(x)+d_{0}(x)\right) f_{0,1}+\left(-\frac{x_{1}}{4} d_{2}(x)\right) f_{0,0}, \\
& \frac{\partial f_{0,2}}{\partial x_{2}}=\operatorname{tr}\left\{\left(\frac{\partial^{2} T_{0}}{\partial x_{1} \partial x_{2}}-\frac{\partial P_{1}}{\partial x_{2}} T_{0}-P_{1} \frac{\partial T_{0}}{\partial x_{2}}\right) P_{0}^{2}+\left(\frac{\partial T_{0}}{\partial x_{1}}-P_{1} T_{0}\right)\left(\frac{\partial P_{0}}{\partial x_{2}} P_{0}+P_{0} \frac{\partial P_{0}}{\partial x_{2}}\right)\right\} \\
& =\operatorname{tr}\left[\frac{\partial}{\partial x_{1}}\left\{-\left[T_{0}, \frac{\partial P_{0}}{\partial x_{1}}\right]+P_{0} \frac{\partial T_{0}}{\partial x_{1}}+\frac{\partial T_{0}}{\partial x_{1}} P_{0}+\left(Q_{1}-\frac{\partial P_{0}}{\partial x_{1}}-P_{0} P_{1}-P_{1} P_{0}\right) T_{0}\right\} P_{0}^{2}\right. \\
& +\left\{\left[P_{2}, Q_{0}\right]+\left[P_{1}, Q_{1}\right]+\left[P_{0}, Q_{2}\right]-\frac{\partial Q_{1}}{\partial x_{1}}\right\} T_{0} P_{0}^{2} \\
& -P_{1}\left\{-\left[T_{0}, \frac{\partial P_{0}}{\partial x_{1}}\right]+P_{0} \frac{\partial T_{0}}{\partial x_{1}}+\frac{\partial T_{0}}{\partial x_{1}} P_{0}+\left(Q_{1}-\frac{\partial P_{0}}{\partial x_{1}}-P_{0} P_{1}-P_{1} P_{0}\right) T_{0}\right\} P_{0}^{2} \\
& \left.+\left(\frac{\partial T_{0}}{\partial x_{1}}-P_{1} T_{0}\right)\left(2 \frac{\partial P_{0}}{\partial x_{1}} P_{0}^{2}+2 P_{0} \frac{\partial P_{0}}{\partial x_{1}} P_{0}\right)\right] \\
& =2 \frac{\partial}{\partial x_{1}} \operatorname{tr}\left\{\left(\frac{\partial T_{0}}{\partial x_{1}}-P_{1} T_{0}\right) P_{0}^{3}\right\} \\
& +\operatorname{tr}\left\{\left(\frac{\partial T_{0}}{\partial x_{1}}-P_{1} T_{0}\right)\left(d_{2}(x) P_{0}^{4}+d_{1}(x) P_{0}^{3}+d_{0}(x) P_{0}^{2}\right)\right\} \\
& =-x_{2} \frac{\partial f_{0,1}}{\partial x_{1}}-\frac{x_{1}}{2} \frac{\partial f_{0,0}}{\partial x_{1}}+\left(-\frac{x_{2}}{2} d_{2}(x)+d_{0}(x)\right) f_{0,2} \\
& +\left(-\frac{x_{1}}{4} d_{2}(x)-\frac{x_{2}}{2} d_{1}(x)\right) f_{0,1}+\left(-\frac{x_{1}}{4} d_{1}(x)-\frac{1}{2}\right) f_{0,0} .
\end{aligned}
$$

Thus we get (4.68). 
Note that the system (4.59) means $f_{0, k}(x)(k=0,1,2)$ vanishes on $\left\{\left(x_{1}, 0\right)\right.$; $\left.x_{1} \neq 0\right\}$. Thus, by Lemmas 4.14 and 4.16 and the holomorphy of $f_{0, k}(x)$, we find that

$$
\left.\left(\begin{array}{l}
f_{0,0} \\
f_{0,1} \\
f_{0,2}
\end{array}\right)\right|_{x_{2}=0}=0
$$

Hence, by the uniqueness of solutions for the initial value problem (4.68) and (4.69), we obtain (4.67).

We have thus obtained a holomorphic solution of (4.48) near $x=0$, that is, we have constructed a $3 \times 3$ matrix $T_{0}(x)$ with holomorphic entries satisfying (4.46). Using it, we now prove Proposition 4.10.

Proof of Proposition 4.10. Let $T_{0}(x)$ be the $3 \times 3$ matrix thus obtained. By (4.60), we have

$$
\operatorname{det} T_{0}(0)=\operatorname{det}\left(c_{0,2}(0) P_{0}(0)^{2}+c_{0,1}(0) P_{0}(0)+c_{0,0}(0)\right)=1 .
$$

Hence, there exists some positive constant $0<\rho<\rho_{0}$ such that $T_{0}(x)$ is holomorphic in $D_{\rho}$ and $\operatorname{det} T_{0}(x) \neq 0\left(x \in D_{\rho}\right)$.

Next, define a $3 \times 3$ matrix $T_{1}(x)$ by

$$
T_{1}=\Lambda\left(\frac{\partial T_{0}}{\partial x_{1}}-P_{1} T_{0}\right)+\Lambda^{2}\left(\frac{\partial T_{0}}{\partial x_{1}}-P_{1} T_{0}\right) P_{0}, \quad \Lambda=\left(\begin{array}{lll}
0 & 0 & 0 \\
1 & 0 & 0 \\
0 & 1 & 0
\end{array}\right)
$$

Then $T_{1}(x)$ is holomorphic in $D_{\rho}$ and it follows from the argument in the proof of Lemma 4.11 that $T_{1}(x)$ satisfies (4.41).

Furthermore, define $3 \times 3$ matrices $\widetilde{P}_{n}(x)$ and $\widetilde{Q}_{n}(x)$ as follows:

$$
\begin{aligned}
& \left\{\begin{array}{l}
\widetilde{P}_{2}=T_{0}^{-1}\left(P_{2} T_{0}+P_{1} T_{1}-\frac{\partial T_{1}}{\partial x_{1}}\right), \\
\widetilde{Q}_{2}=T_{0}^{-1}\left(Q_{2} T_{0}+Q_{1} T_{1}-T_{1} \frac{\partial P_{0}}{\partial x_{1}}-\frac{\partial T_{1}}{\partial x_{2}}\right),
\end{array}\right. \\
& \left\{\begin{array}{l}
\widetilde{P}_{n}=T_{0}^{-1}\left(P_{n} T_{0}-T_{1} \widetilde{P}_{n-1}+P_{n-1} T_{1}\right), \quad(n \geq 3) . \\
\widetilde{Q}_{n}=T_{0}^{-1}\left(Q_{n} T_{0}-T_{1} \widetilde{Q}_{n-1}+Q_{n-1} T_{1}\right), \quad
\end{array}\right.
\end{aligned}
$$

These matrices are holomorphic in $D_{\rho}$ and satisfy (4.42) and (4.43). 
Finally, we prove the estimate $(4.39)$ for $\widetilde{P}_{n}(x)$ and $\widetilde{Q}_{n}(x)$. Since $T_{i}(x)(i=$ $0,1), T_{0}(x)^{-1}, \widetilde{P}_{i}(x)(i=0,1,2)$ and $\widetilde{Q}_{i}(x)(i=0,1,2)$ are holomorphic in $D_{\rho}$, we have

$$
\left\|T_{i}\right\|_{\rho, \rho},\left\|T_{0}^{-1}\right\|_{\rho, \rho},\left\|\widetilde{P}_{i}\right\|_{\rho, \rho},\left\|\widetilde{Q}_{i}\right\|_{\rho, \rho} \leq C^{\dagger}
$$

with some positive constant $C^{\dagger}>0$. Then, if we define positive constants $\widetilde{C}$ and $\widetilde{\alpha}$ by

$$
\widetilde{C}=\max \left\{C^{\dagger}, C(\alpha+1)\right\}, \quad \widetilde{\alpha}=\max \left\{1, \alpha, 2\left(C^{\dagger}\right)^{2}\right\},
$$

the estimate (4.39) holds. In fact, we have

$$
\begin{aligned}
&\left\|\widetilde{P}_{0}\right\|_{\rho, \rho},\left\|\widetilde{Q}_{0}\right\|_{\rho, \rho} \leq C^{\dagger} \leq \widetilde{C} \\
&\left\|\widetilde{P}_{1}\right\|_{\rho, \rho},\left\|\widetilde{Q}_{1}\right\|_{\rho, \rho} \leq C^{\dagger} \leq \widetilde{C} \widetilde{\alpha} \\
&\left\|\widetilde{P}_{2}\right\|_{\rho, \rho},\left\|\widetilde{Q}_{2}\right\|_{\rho, \rho} \leq C^{\dagger} \leq \widetilde{C} \widetilde{\alpha}^{2} 2 !
\end{aligned}
$$

and, using the definition (4.70) of $\widetilde{P}_{n}(x)$ and $\widetilde{Q}_{n}(x)$ and induction on $n \geq 3$, we obtain

$$
\begin{aligned}
\left\|\widetilde{P}_{n}\right\|_{\rho, \rho},\left\|\widetilde{Q}_{n}\right\|_{\rho, \rho} & \leq\left(C^{\dagger}\right)^{2}\left(C \alpha^{n} n !+\widetilde{C} \widetilde{\alpha}^{n-1}(n-1) !+C \alpha^{n-1}(n-1) !\right) \\
& \leq \frac{\widetilde{\alpha}}{2}(C \alpha+\widetilde{C}+C) \widetilde{\alpha}^{n-1} n ! \leq \widetilde{C} \widetilde{\alpha}^{n} n !
\end{aligned}
$$

\section{$\S 4.3$. Construction of the transformation, III}

Thanks to the argument so far, we may assume that the completely integrable system in question has the form

$$
\begin{cases}\eta^{-1} \frac{\partial}{\partial x_{1}} \Psi=P(x, \eta) \Psi, & P(x, \eta)=\sum_{n \geq 0} \eta^{-n} P_{n}(x) \\ \eta^{-1} \frac{\partial}{\partial x_{2}} \Psi=Q(x, \eta) \Psi, & Q(x, \eta)=\sum_{n \geq 0} \eta^{-n} Q_{n}(x) .\end{cases}
$$

Here

$$
P_{0}=\left(\begin{array}{ccc}
0 & 1 & 0 \\
0 & 0 & 1 \\
-x_{1} / 4 & -x_{2} / 2 & 0
\end{array}\right), \quad P_{1}=0, \quad Q_{0}=P_{0}^{2}+\frac{x_{2}}{3}, \quad Q_{1}=\frac{\partial P_{0}}{\partial x_{1}}
$$

and $P_{n}(x)$ and $Q_{n}(x)$ are $3 \times 3$ matrices with holomorphic entries in $D_{\rho}$ satisfying

$$
\left\|P_{n}\right\|_{\rho, \rho},\left\|Q_{n}\right\|_{\rho, \rho} \leq C \alpha^{n} n ! \quad(n \geq 0)
$$

with some positive constants $C$ and $\alpha$. 
In this subsection, we prove

Proposition 4.18. There exists an infinite sequence $\left\{T_{n}(x)\right\}_{n \geq 0}$ of $3 \times 3$ matrices with the following properties:

- Every entry of $T_{n}(x)$ is holomorphic in $D_{\rho}$ and $\operatorname{det} T_{0}(x) \neq 0\left(x \in D_{\rho}\right)$.

- $T_{n}(x)$ satisfies

$$
\left\|T_{n}\right\|_{\rho, \rho} \leq \widetilde{C} \widetilde{\alpha}^{n} n !
$$

with some positive constants $\widetilde{C}$ and $\widetilde{\alpha}$.

- The formal transformation

$$
\Psi(x, \eta)=T(x, \eta) \widetilde{\Psi}(x, \eta), \quad T(x, \eta)=\sum_{n \geq 0} \eta^{-n} T_{n}(x),
$$

transforms (4.71) into

$$
\begin{cases}\eta^{-1} \frac{\partial}{\partial x_{1}} \widetilde{\Psi}=\widetilde{P}(x, \eta) \widetilde{\Psi}, & \widetilde{P}(x, \eta)=\sum_{n \geq 0} \eta^{-n} \widetilde{P}_{n}(x), \\ \eta^{-1} \frac{\partial}{\partial x_{2}} \widetilde{\Psi}=\widetilde{Q}(x, \eta) \widetilde{\Psi}, & \widetilde{Q}(x, \eta)=\sum_{n \geq 0} \eta^{-n} \widetilde{Q}_{n}(x),\end{cases}
$$

where

$$
\begin{aligned}
& \widetilde{P}_{0}=P_{0}, \quad \widetilde{P}_{n}=0 \quad(n \geq 1), \\
& \widetilde{Q}_{0}=Q_{0}, \quad \widetilde{Q}_{1}=Q_{1}, \quad \widetilde{Q}_{n}=0 \quad(n \geq 2) .
\end{aligned}
$$

In this subsection we only discuss the construction of $\left\{T_{n}(x)\right\}_{n \geq 0}$. The estimate (4.74) will be proved in the next section.

Since the system (4.76) is obtained from (4.71) through the transformation (4.75), $T(x, \eta)$ should satisfy

$$
\left\{\begin{array}{l}
T \widetilde{P}+\eta^{-1} \frac{\partial T}{\partial x_{1}}=P T \\
T \widetilde{Q}+\eta^{-1} \frac{\partial T}{\partial x_{2}}=Q T
\end{array}\right.
$$

that is,

$$
\left\{\begin{array}{l}
{\left[P_{0}, T_{n}\right]=F_{n, 1}, \quad(n \geq 0),} \\
{\left[Q_{0}, T_{n}\right]=F_{n, 2},}
\end{array}\right.
$$

where

$$
F_{0,1}=F_{0,2}=0
$$




$$
\begin{aligned}
& \left\{\begin{array}{l}
F_{1,1}=\frac{\partial T_{0}}{\partial x_{1}}, \\
F_{1,2}=\frac{\partial T_{0}}{\partial x_{2}}+\left[T_{0}, \frac{\partial P_{0}}{\partial x_{1}}\right],
\end{array}\right. \\
& \left\{\begin{array}{l}
F_{n, 1}=\frac{\partial T_{n-1}}{\partial x_{1}}-\sum_{i=0}^{n-2} P_{n-i} T_{i}, \\
F_{n, 2}=\frac{\partial T_{n-1}}{\partial x_{2}}-\sum_{i=0}^{n-2} Q_{n-i} T_{i}+\left[T_{n-1}, \frac{\partial P_{0}}{\partial x_{1}}\right],
\end{array} \quad(n \geq 2) .\right.
\end{aligned}
$$

We now discuss the construction of $\left\{T_{n}(x)\right\}_{n \geq 0}$ satisfying the relations $\left(E_{n}\right)$. Having Lemma 4.11 in mind, we prove the following assertions $\left(\mathcal{S}_{n}\right)(n=0,1,2, \ldots)$ by induction on $n$ :

$\left(\mathcal{S}_{n}\right)$ There exist $3 \times 3$ matrices $T_{i}(x)$ for $i=0,1, \ldots, n$ that satisfy:

- $T_{i}(x)(i=0,1, \ldots, n)$ are holomorphic in $D_{\rho}$.

- $\left(E_{j}\right)$ holds for $j=0,1, \ldots, n$.

- $\operatorname{tr}\left(F_{n+1,1} P_{0}^{k}\right)=0(k=0,1,2)$.

- $F_{n+1,2}=P_{0} F_{n+1,1}+F_{n+1,1} P_{0}$.

We trivially have $\left(\mathcal{S}_{0}\right)$ by setting $T_{0}(x)=I$. Let us prove $\left(\mathcal{S}_{n}\right)$ provided that $\left(\mathcal{S}_{n-1}\right)$ holds. For this purpose it suffices to construct $T_{n}(x)$ such that:

- $T_{n}(x)$ is holomorphic in $D_{\rho}$.

- $\left(E_{n}\right)$ holds.

- $\operatorname{tr}\left(F_{n+1,1} P_{0}^{k}\right)=0(k=0,1,2)$.

- $F_{n+1,2}=P_{0} F_{n+1,1}+F_{n+1,1} P_{0}$.

It follows from the induction hypothesis that $F_{n, 1}$ is holomorphic in $D_{\rho}$ and

$$
\left\{\begin{array}{l}
\operatorname{tr}\left(F_{n, 1} P_{0}^{k}\right)=0 \quad(k=0,1,2), \\
F_{n, 2}=P_{0} F_{n, 1}+F_{n, 1} P_{0} .
\end{array}\right.
$$

We now define a $3 \times 3$ matrix $T_{n}(x)$ as follows:

$$
\begin{aligned}
T_{n} & =T_{n, 1}+T_{n, 2}, \\
T_{n, 1} & =c_{n, 2}(x) P_{0}^{2}+c_{n, 1}(x) P_{0}+c_{n, 0}(x), \\
T_{n, 2} & =\Lambda F_{n, 1}+\Lambda^{2} F_{n, 1} P_{0},
\end{aligned}
$$

where $c_{n, k}(x)(k=0,1,2)$ are holomorphic functions in $D_{\rho}$ which will be determined later. Then $T_{n}(x)$ is holomorphic in $D_{\rho}$ and satisfies

$$
\left[P_{0}, T_{n}\right]=\left[P_{0}, T_{n, 2}\right]=F_{n, 1},
$$




$$
\left[Q_{0}, T_{n}\right]=\left[Q_{0}, T_{n, 2}\right]=F_{n, 2}
$$

by the same argument as in the proof of Lemma 4.11. Hence $\left(E_{n}\right)$ is satisfied. Thus, in what follows, we determine holomorphic functions $c_{n, k}(x)(k=0,1,2)$ in $D_{\rho}$ so that

$$
\left\{\begin{array}{l}
\operatorname{tr}\left(F_{n+1,1} P_{0}^{k}\right)=0 \quad(k=0,1,2), \\
F_{n+1,2}=P_{0} F_{n+1,1}+F_{n+1,1} P_{0} .
\end{array}\right.
$$

Let $F_{n+1,1}^{\dagger}$ and $F_{n+1,2}^{\dagger}$ be the $3 \times 3$ matrices defined by

$$
\begin{aligned}
& F_{n+1,1}^{\dagger}=F_{n+1,1}-\frac{\partial T_{n, 1}}{\partial x_{1}}, \\
& F_{n+1,2}^{\dagger}=F_{n+1,2}-\frac{\partial T_{n, 1}}{\partial x_{2}}-\left[T_{n, 1}, \frac{\partial P_{0}}{\partial x_{1}}\right]
\end{aligned}
$$

that is, $F_{n+1,1}^{\dagger}$ (resp., $F_{n+1,2}^{\dagger}$ ) is the part of $F_{n+1,1}$ (resp., $F_{n+1,2}$ ) which is independent of $T_{n, 1}$. For these matrices, we have

Sublemma 4.19. There exist holomorphic functions $g_{n, k}(x)(k=0,1,2)$ in $D_{\rho}$ which satisfy

$$
-F_{n+1,2}^{\dagger}+F_{n+1,1}^{\dagger} P_{0}+P_{0} F_{n+1,1}^{\dagger}=g_{n, 2}(x) P_{0}^{2}+g_{n, 1}(x) P_{0}+g_{n, 0}(x) .
$$

Proof. By the definition,

$$
\begin{aligned}
& F_{n+1,1}^{\dagger}=\frac{\partial T_{n, 2}}{\partial x_{1}}-\sum_{i=0}^{n-1} P_{n+1-i} T_{i} \\
& F_{n+1,2}^{\dagger}=\frac{\partial T_{n, 2}}{\partial x_{2}}-\sum_{i=0}^{n-1} Q_{n+1-i} T_{i}+\left[T_{n, 2}, \frac{\partial P_{0}}{\partial x_{1}}\right] .
\end{aligned}
$$

Using the compatibility condition, $\left(E_{j}\right)(j=0,1, \ldots, n-1),(4.77)$ and (4.78), we have

$$
\begin{aligned}
{[-} & \left.F_{n+1,2}^{\dagger}+F_{n+1,1}^{\dagger} P_{0}+P_{0} F_{n+1,1}^{\dagger}, P_{0}\right] \\
= & {\left[P_{0}, \frac{\partial T_{n, 2}}{\partial x_{2}}\right]+\left[\frac{\partial T_{n, 2}}{\partial x_{1}}, Q_{0}\right]+P_{0}\left[T_{n, 2}, \frac{\partial P_{0}}{\partial x_{1}}\right]-\left[T_{n, 2}, \frac{\partial P_{0}}{\partial x_{1}}\right] P_{0} } \\
& \quad-\sum_{i=0}^{n-1} P_{0} Q_{n+1-i} T_{i}+\sum_{i=0}^{n-1} Q_{0} P_{n+1-i} T_{i}-\sum_{i=0}^{n-1} P_{n+1-i} T_{i} Q_{0}+\sum_{i=0}^{n-1} Q_{n+1-i} T_{i} P_{0}
\end{aligned}
$$




$$
\begin{aligned}
& =\left(-\left[\frac{\partial P_{0}}{\partial x_{2}}, T_{n, 2}\right]+\frac{\partial^{2} T_{n-1}}{\partial x_{1} \partial x_{2}}-\sum_{i=0}^{n-2} \frac{\partial P_{n-i}}{\partial x_{2}} T_{i}-\sum_{i=0}^{n-2} P_{n-i} \frac{\partial T_{i}}{\partial x_{2}}\right) \\
& +\left(\left[\frac{\partial Q_{0}}{\partial x_{1}}, T_{n, 2}\right]-\frac{\partial^{2} T_{n-1}}{\partial x_{1} \partial x_{2}}+\sum_{i=0}^{n-2} \frac{\partial Q_{n-i}}{\partial x_{1}} T_{i}+\sum_{i=0}^{n-2} Q_{n-i} \frac{\partial T_{i}}{\partial x_{1}}-\left[\frac{\partial T_{n-1}}{\partial x_{1}}, \frac{\partial P_{0}}{\partial x_{1}}\right]\right) \\
& +P_{0}\left[T_{n, 2}, \frac{\partial P_{0}}{\partial x_{1}}\right]-\left[T_{n, 2}, \frac{\partial P_{0}}{\partial x_{1}}\right] P_{0}-\sum_{i=0}^{n-1} P_{0} Q_{n+1-i} T_{i}+\sum_{i=0}^{n-1} Q_{0} P_{n+1-i} T_{i} \\
& +\left(-\sum_{i=0}^{n-1} P_{n+1-i} Q_{0} T_{i}+\sum_{i=0}^{n-2} P_{n-i} \frac{\partial T_{i}}{\partial x_{2}}+\sum_{i=0}^{n-2} P_{n-i}\left[T_{i}, \frac{\partial P_{0}}{\partial x_{1}}\right]\right. \\
& \left.-\sum_{i=2}^{n-1} \sum_{k=0}^{i-2} P_{n+1-i} Q_{i-k} T_{k}\right) \\
& +\left(\sum_{i=0}^{n-1} Q_{n+1-i} P_{0} T_{i}-\sum_{i=0}^{n-2} Q_{n-i} \frac{\partial T_{i}}{\partial x_{1}}+\sum_{i=2}^{n-1} \sum_{k=0}^{i-2} Q_{n+1-i} P_{i-k} T_{k}\right) \\
& =\left[T_{n, 2}, \frac{\partial P_{0}}{\partial x_{2}}-\frac{\partial Q_{0}}{\partial x_{1}}+\left[P_{0}, \frac{\partial P_{0}}{\partial x_{1}}\right]\right]+\left[\left[P_{0}, T_{n, 2}\right]-\frac{\partial T_{n-1}}{\partial x_{1}}, \frac{\partial P_{0}}{\partial x_{1}}\right] \\
& -\sum_{i=0}^{n-2}\left(\frac{\partial P_{n-i}}{\partial x_{2}}-\frac{\partial Q_{n-i}}{\partial x_{1}}\right) T_{i}-\sum_{i=0}^{n-1}\left(\left[P_{0}, Q_{n+1-i}\right]+\left[P_{n+1-i}, Q_{0}\right]\right) T_{i} \\
& +\sum_{i=0}^{n-2} P_{n-i}\left[T_{i}, \frac{\partial P_{0}}{\partial x_{1}}\right]-\sum_{i=2}^{n-1} \sum_{k=2}^{i-2}\left(P_{n+1-i} Q_{i-k}-Q_{n+1-i} P_{i-k}\right) T_{k} \\
& =\left[\left[P_{0}, T_{n, 2}\right]-\frac{\partial T_{n-1}}{\partial x_{1}}, \frac{\partial P_{0}}{\partial x_{1}}\right]+\sum_{i=0}^{n-2} \sum_{k=0}^{n+1-i}\left[P_{k}, Q_{n+1-i-k}\right] T_{i} \\
& -\sum_{i=0}^{n-1}\left(\left[P_{0}, Q_{n+1-i}\right]+\left[P_{n+1-i}, Q_{0}\right]\right) T_{i}+\sum_{i=0}^{n-2} P_{n-i}\left[T_{i}, \frac{\partial P_{0}}{\partial x_{1}}\right] \\
& -\sum_{i=0}^{n-3} \sum_{k=2}^{n-i-1}\left[P_{k}, Q_{n+1-i-k}\right] T_{i} \\
& =\left[-\sum_{i=0}^{n-2} P_{n-i} T_{i}, \frac{\partial P_{0}}{\partial x_{1}}\right]+\sum_{i=0}^{n-2} P_{n-i}\left[T_{i}, \frac{\partial P_{0}}{\partial x_{1}}\right]+\sum_{i=0}^{n-2}\left[P_{n-i}, Q_{1}\right] T_{i} \\
& =-\sum_{i=0}^{n-2}\left[P_{n-i}, \frac{\partial P_{0}}{\partial x_{1}}\right] T_{i}+\sum_{i=0}^{n-2}\left[P_{n-i}, Q_{1}\right] T_{i}=0 \text {. }
\end{aligned}
$$

Hence, by the same argument as in the proof of Lemma 2.4, we get (4.80).

Using this lemma, we find

Lemma 4.20. The relation (4.79) implies that $\left(c_{n, 0}(x), c_{n, 1}(x), c_{n, 2}(x)\right)$ satisfies the system of partial differential equations 


$$
\left\{\begin{array}{l}
\left(3 x_{1}+4 x_{2} P_{0}^{T}\right) \frac{\partial}{\partial x_{1}}\left(\begin{array}{l}
c_{n, 0} \\
c_{n, 1} \\
c_{n, 2}
\end{array}\right)=\operatorname{diag}(0,-1,-2)\left(\begin{array}{l}
c_{n, 0} \\
c_{n, 1} \\
c_{n, 2}
\end{array}\right)+\left(\begin{array}{c}
-x_{1} h_{n, 0}(x) \\
4 h_{n, 1}(x) \\
4 h_{n, 2}(x)
\end{array}\right) \\
\frac{\partial}{\partial x_{2}}\left(\begin{array}{l}
c_{n, 0} \\
c_{n, 1} \\
c_{n, 2}
\end{array}\right)=2 P_{0}^{T} \frac{\partial}{\partial x_{1}}\left(\begin{array}{l}
c_{n, 0} \\
c_{n, 1} \\
c_{n, 2}
\end{array}\right)+\left(\begin{array}{l}
g_{n, 0}(x) \\
g_{n, 1}(x) \\
g_{n, 2}(x)
\end{array}\right)
\end{array}\right.
$$

where

$$
h_{n, 0}(x)=\operatorname{tr}\left(F_{n+1,1}^{\dagger}\right), \quad h_{n, 1}(x)=\operatorname{tr}\left(F_{n+1,1}^{\dagger} P_{0}^{2}\right), \quad h_{n, 2}(x)=\operatorname{tr}\left(F_{n+1,1}^{\dagger} P_{0}\right) .
$$

Conversely, if $\left(c_{n, 0}(x), c_{n, 1}(x), c_{n, 2}(x)\right)$ is a holomorphic solution of (4.81), then the relation (4.79) holds.

Proof. First, we consider

$$
\operatorname{tr}\left(F_{n+1,1} P_{0}^{k}\right)=0 \quad(k=0,1,2) .
$$

By the relations (4.49), we obtain

$$
\begin{aligned}
\operatorname{tr}\left(F_{n+1,1}\right) & =-x_{2} \frac{\partial c_{n, 2}}{\partial x_{1}}+3 \frac{\partial c_{n, 0}}{\partial x_{1}}+h_{n, 0}(x), \\
\operatorname{tr}\left(F_{n+1,1} P_{0}\right) & =-\frac{3}{4} x_{1} \frac{\partial c_{n, 2}}{\partial x_{1}}-x_{2} \frac{\partial c_{n, 1}}{\partial x_{1}}-\frac{1}{2} c_{n, 2}+h_{n, 2}(x), \\
\operatorname{tr}\left(F_{n+1,1} P_{0}^{2}\right) & =\frac{x_{2}^{2}}{2} \frac{\partial c_{n, 2}}{\partial x_{1}}-\frac{3}{4} x_{1} \frac{\partial c_{n, 1}}{\partial x_{1}}-x_{2} \frac{\partial c_{n, 0}}{\partial x_{1}}-\frac{1}{4} c_{n, 1}+h_{n, 1}(x) .
\end{aligned}
$$

If we multiply (4.82) (resp., (4.83), (4.84)) by $x_{1}$ (resp., $(-4),(-4)$ ), we get the first equation of (4.81).

Next, we consider

$$
F_{n+1,2}=P_{0} F_{n+1,1}+F_{n+1,1} P_{0} .
$$

By (4.80), this is equivalent to

$$
\begin{aligned}
\frac{\partial T_{n, 1}}{\partial x_{2}}-\left[\frac{\partial P_{0}}{\partial x_{1}}, T_{n, 1}\right]-P_{0} \frac{\partial T_{n, 1}}{\partial x_{1}} & -\frac{\partial T_{n, 1}}{\partial x_{1}} P_{0} \\
& =g_{n, 2}(x) P_{0}^{2}+g_{n, 1}(x) P_{0}+g_{n, 0}(x) .
\end{aligned}
$$

By (4.55) and (4.56), we have

$$
\begin{aligned}
& \frac{\partial T_{n, 1}}{\partial x_{2}}+\left[T_{n, 1}, \frac{\partial P_{0}}{\partial x_{1}}\right]-P_{0} \frac{\partial T_{n, 1}}{\partial x_{1}}-\frac{\partial T_{n, 1}}{\partial x_{1}} P_{0} \\
& =\left(\frac{\partial c_{n, 2}}{\partial x_{2}}-2 \frac{\partial c_{n, 1}}{\partial x_{1}}\right) P_{0}^{2}+\left(\frac{\partial c_{n, 1}}{\partial x_{2}}+x_{2} \frac{\partial c_{n, 2}}{\partial x_{1}}-2 \frac{\partial c_{n, 0}}{\partial x_{1}}\right) P_{0}+\left(\frac{\partial c_{n, 0}}{\partial x_{2}}+\frac{x_{1}}{2} \frac{\partial c_{n, 2}}{\partial x_{1}}\right) .
\end{aligned}
$$

Comparing the left-hand and right-hand sides of (4.85), we obtain

$$
\frac{\partial c_{n, 2}}{\partial x_{2}}-2 \frac{\partial c_{n, 1}}{\partial x_{1}}=g_{n, 2}(x),
$$




$$
\begin{aligned}
\frac{\partial c_{n, 1}}{\partial x_{2}}+x_{2} \frac{\partial c_{n, 2}}{\partial x_{1}}-2 \frac{\partial c_{n, 0}}{\partial x_{1}} & =g_{n, 1}(x) \\
\frac{\partial c_{n, 0}}{\partial x_{2}}+\frac{x_{1}}{2} \frac{\partial c_{n, 2}}{\partial x_{1}} & =g_{n, 0}(x)
\end{aligned}
$$

Thus we also get the second equation of (4.81).

The "converse" part is clear from the above argument.

We now prove the existence of a holomorphic solution of the system (4.81).

Lemma 4.21. Let

$$
3 x_{1} \frac{d}{d x_{1}}\left(\begin{array}{c}
c_{n, 0} \\
c_{n, 1} \\
c_{n, 2}
\end{array}\right)=\operatorname{diag}(0,-1,-2)\left(\begin{array}{c}
c_{n, 0} \\
c_{n, 1} \\
c_{n, 2}
\end{array}\right)+\left.\left(\begin{array}{c}
-x_{1} h_{n, 0}(x) \\
4 h_{n, 1}(x) \\
4 h_{n, 2}(x)
\end{array}\right)\right|_{x_{2}=0}
$$

be the system of ordinary differential equation obtained by restricting the first equation of (4.81) to $x_{2}=0$. Then (4.86) has a holomorphic solution $\left(c_{n, 0}\left(x_{1}, 0\right), c_{n, 1}\left(x_{1}, 0\right), c_{n, 2}\left(x_{1}, 0\right)\right)$ near $x_{1}=0$ satisfying

$$
c_{n, 0}(0)=0
$$

Proof. By the method of variation of constants, we find that

$$
\left\{\begin{array}{l}
c_{n, 0}\left(x_{1}, 0\right)=\frac{1}{3} \int_{0}^{1}\left\{-x_{1} h_{n, 0}\left(x_{1} s, 0\right)\right\} d s \\
c_{n, 1}\left(x_{1}, 0\right)=\frac{1}{3} \int_{0}^{1}\left\{4 s^{-2 / 3} h_{n, 1}\left(x_{1} s, 0\right)\right\} d s \\
c_{n, 2}\left(x_{1}, 0\right)=\frac{1}{3} \int_{0}^{1}\left\{4 s^{-1 / 3} h_{n, 2}\left(x_{1} s, 0\right)\right\} d s
\end{array}\right.
$$

is a holomorphic solution of the system (4.86) satisfying (4.87).

Lemma 4.22. Let $\left(c_{n, 0}\left(x_{1}, 0\right), c_{n, 1}\left(x_{1}, 0\right), c_{n, 2}\left(x_{1}, 0\right)\right)$ be a holomorphic solution near $x_{1}=0$ given in Lemma 4.21. Then the initial value problem

$$
\left\{\begin{array}{l}
\frac{\partial}{\partial x_{2}}\left(\begin{array}{l}
c_{n, 0} \\
c_{n, 1} \\
c_{n, 2}
\end{array}\right)=2 P_{0}^{T} \frac{\partial}{\partial x_{1}}\left(\begin{array}{l}
c_{n, 0} \\
c_{n, 1} \\
c_{n, 2}
\end{array}\right)+\left(\begin{array}{l}
g_{n, 0}(x) \\
g_{n, 1}(x) \\
g_{n, 2}(x)
\end{array}\right) \\
\left.\left(\begin{array}{l}
c_{n, 0} \\
c_{n, 1} \\
c_{n, 2}
\end{array}\right)\right|_{x_{2}=0}=\left(\begin{array}{l}
c_{n, 0}\left(x_{1}, 0\right) \\
c_{n, 1}\left(x_{1}, 0\right) \\
c_{n, 2}\left(x_{1}, 0\right)
\end{array}\right)
\end{array}\right.
$$

has a holomorphic solution near $x=0$.

This lemma immediately follows from the Cauchy-Kowalevski theorem.

Furthermore, we can prove 
Lemma 4.23. The holomorphic solution $\left(c_{n, 0}(x), c_{n, 1}(x), c_{n, 2}(x)\right)$ given by Lemma 4.22 satisfies the first equation of (4.81).

In the proof of this lemma we use

Sublemma 4.24. For $k=0,1,2$, we have

$$
\begin{aligned}
\operatorname{tr}\left[\left\{-\frac{\partial}{\partial x_{2}}\left(\sum_{i=0}^{n-1} P_{n+1-i} T_{i}\right)+\frac{\partial}{\partial x_{1}}\right.\right. & \left.\left.\left(\sum_{i=0}^{n-1} Q_{n+1-i} T_{i}\right)\right\} P_{0}^{k}\right] \\
& =\operatorname{tr}\left\{\frac{\partial P_{0}}{\partial x_{1}}\left[P_{0}^{k}, \sum_{i=0}^{n-1} P_{n+1-i} T_{i}\right]\right\} .
\end{aligned}
$$

Proof. By the compatibility condition, (4.72) and $\left(E_{j}\right)(j=0,1, \ldots, n)$, we have

$$
\begin{aligned}
& \operatorname{tr}\left\{\sum_{i=0}^{n}\left(-\frac{\partial P_{n+1-i}}{\partial x_{2}}+\frac{\partial Q_{n+1-i}}{\partial x_{1}}\right) T_{i} P_{0}^{k}\right\}=\operatorname{tr}\left(\sum_{i=0}^{n} \sum_{j=0}^{n+2-i}\left[P_{j}, Q_{n+2-i-j}\right] T_{i} P_{0}^{k}\right) \\
& =\operatorname{tr}\left\{\sum_{i=0}^{n}\left(\sum_{j=1}^{n+1-i}\left[P_{j}, Q_{n+2-i-j}\right] T_{i}+P_{n+2-i}\left[Q_{0}, T_{i}\right]-Q_{n+2-i}\left[P_{0}, T_{i}\right]\right) P_{0}^{k}\right\} \\
& \operatorname{tr}\left(\sum_{i=0}^{n} \sum_{j=1}^{n+1-i}\left[P_{j}, Q_{n+2-i-j}\right] T_{i} P_{0}^{k}\right) \\
& +\operatorname{tr}\left[\sum _ { i = 1 } ^ { n } \left\{P_{n+2-i}\left(\frac{\partial T_{i-1}}{\partial x_{2}}-\sum_{j=0}^{i-1} Q_{i-j} T_{j}+T_{i-1} \frac{\partial P_{0}}{\partial x_{1}}\right)\right.\right. \\
& \left.\left.-Q_{n+2-i}\left(\frac{\partial T_{i-1}}{\partial x_{1}}-\sum_{j=0}^{i-1} P_{i-j} T_{j}\right)\right\} P_{0}^{k}\right] \\
& \operatorname{tr}\left(\sum_{i=0}^{n-1} \sum_{j=2}^{n+1-i}\left[P_{j}, Q_{n+2-i-j}\right] T_{i} P_{0}^{k}\right) \\
& +\operatorname{tr}\left\{\sum_{i=1}^{n}\left(P_{n+2-i} \frac{\partial T_{i-1}}{\partial x_{2}}+P_{n+2-i} T_{i-1} \frac{\partial P_{0}}{\partial x_{1}}-Q_{n+2-i} \frac{\partial T_{i-1}}{\partial x_{1}}\right) P_{0}^{k}\right\} \\
& +\operatorname{tr}\left\{\sum_{i=0}^{n-1}\left(\sum_{j=2}^{n-i}\left[Q_{n+2-i-j}, P_{j}\right]-P_{n+1-i} Q_{1}\right) T_{i} P_{0}^{k}\right\} \\
& =\operatorname{tr}\left(\sum_{i=0}^{n-1}\left[P_{n+1-i}, Q_{1}\right] T_{i} P_{0}^{k}\right) \\
& +\operatorname{tr}\left\{\sum_{i=0}^{n-1}\left(P_{n+1-i} \frac{\partial T_{i}}{\partial x_{2}}+P_{n+1-i} T_{i} \frac{\partial P_{0}}{\partial x_{1}}-Q_{n+1-i} \frac{\partial T_{i}}{\partial x_{1}}\right) P_{0}^{k}\right\} \\
& -\operatorname{tr}\left(\sum_{i=0}^{n-1} P_{n+1-i} Q_{1} T_{i} P_{0}^{k}\right)
\end{aligned}
$$




$$
\begin{aligned}
= & \operatorname{tr}\left\{\sum_{i=0}^{n-1}\left(P_{n+1-i} \frac{\partial T_{i}}{\partial x_{2}}-Q_{n+1-i} \frac{\partial T_{i}}{\partial x_{1}}\right) P_{0}^{k}\right\} \\
& +\operatorname{tr}\left\{\sum_{i=0}^{n-1}\left(P_{n+1-i} T_{i} \frac{\partial P_{0}}{\partial x_{1}}-Q_{1} P_{n+1-i} T_{i}\right) P_{0}^{k}\right\} \\
= & \operatorname{tr}\left\{\sum_{i=0}^{n-1}\left(P_{n+1-i} \frac{\partial T_{i}}{\partial x_{2}}-Q_{n+1-i} \frac{\partial T_{i}}{\partial x_{1}}\right) P_{0}^{k}\right\}+\operatorname{tr}\left\{\frac{\partial P_{0}}{\partial x_{1}}\left[P_{0}^{k}, \sum_{i=0}^{n-1} P_{n+1-i} T_{i}\right]\right\} .
\end{aligned}
$$

Proof of Lemma 4.23. By the argument of Lemma 4.20, it suffices to prove that

$$
T_{n, 1}=c_{n, 2}(x) P_{0}^{2}+c_{n, 1}(x) P_{0}+c_{n, 0}(x)
$$

satisfies

$$
\operatorname{tr}\left(F_{n+1,1} P_{0}^{k}\right)=0 \quad(k=0,1,2) .
$$

Let $f_{n, k}(x)(k=0,1,2)$ be holomorphic functions defined by

$$
f_{n, k}=\operatorname{tr}\left(F_{n+1,1} P_{0}^{k}\right) \quad(k=0,1,2) .
$$

We will prove that $\left(f_{n, 0}(x), f_{n, 1}(x), f_{n, 2}(x)\right)$ satisfies the system of partial differential equations

$$
\frac{\partial}{\partial x_{2}}\left(\begin{array}{l}
f_{n, 0} \\
f_{n, 1} \\
f_{n, 2}
\end{array}\right)=2 \frac{\partial}{\partial x_{1}}\left\{P_{0}\left(\begin{array}{l}
f_{n, 0} \\
f_{n, 1} \\
f_{n, 2}
\end{array}\right)\right\} .
$$

By the second equation of (4.79), we have

$$
\begin{aligned}
\operatorname{tr}\left(F_{n+1,2}\right) & =2 \operatorname{tr}\left(F_{n+1,1} P_{0}\right)=2 f_{n, 1}, \\
\operatorname{tr}\left(F_{n+1,2} P_{0}\right) & =2 \operatorname{tr}\left(F_{n+1,1} P_{0}^{2}\right)=2 f_{n, 2}, \\
\operatorname{tr}\left(F_{n+1,2} P_{0}^{2}\right) & =2 \operatorname{tr}\left(F_{n+1,1} P_{0}^{3}\right)=-x_{2} f_{n, 1}-\frac{x_{1}}{2} f_{n, 0},
\end{aligned}
$$

that is,

$$
\left(\begin{array}{c}
\operatorname{tr}\left(F_{n+1,2}\right) \\
\operatorname{tr}\left(F_{n+1,2} P_{0}\right) \\
\operatorname{tr}\left(F_{n+1,2} P_{0}^{2}\right)
\end{array}\right)=2 P_{0}\left(\begin{array}{l}
f_{n, 0} \\
f_{n, 1} \\
f_{n, 2}
\end{array}\right) .
$$

Hence (4.91) is equivalent to

$$
\frac{\partial}{\partial x_{2}}\left\{\operatorname{tr}\left(F_{n+1,1} P_{0}^{k}\right)\right\}=\frac{\partial}{\partial x_{1}}\left\{\operatorname{tr}\left(F_{n+1,2} P_{0}^{k}\right)\right\} \quad(k=0,1,2) .
$$

By using Sublemma 4.24, we can confirm these relations as follows: 


$$
\begin{aligned}
& \frac{\partial}{\partial x_{2}}\left\{\operatorname{tr}\left(F_{n+1,1}\right)\right\}-\frac{\partial}{\partial x_{1}}\left\{\operatorname{tr}\left(F_{n+1,2}\right)\right\}=\operatorname{tr}\left(\frac{\partial F_{n+1,1}}{\partial x_{2}}-\frac{\partial F_{n+1,2}}{\partial x_{1}}\right) \\
& =\operatorname{tr}\left[\left\{\frac{\partial^{2} T_{n}}{\partial x_{2} \partial x_{1}}-\frac{\partial}{\partial x_{2}}\left(\sum_{i=0}^{n-1} P_{n+1-i} T_{i}\right)\right\}\right. \\
& \left.-\left\{\frac{\partial^{2} T_{n}}{\partial x_{1} \partial x_{2}}-\frac{\partial}{\partial x_{1}}\left(\sum_{i=0}^{n-1} Q_{n+1-i} T_{i}\right)+\left[\frac{\partial T_{n}}{\partial x_{1}}, \frac{\partial P_{0}}{\partial x_{1}}\right]\right\}\right] \\
& =\operatorname{tr}\left\{\frac{\partial P_{0}}{\partial x_{1}}\left[I, \sum_{i=0}^{n-1} P_{n+1-i} T_{i}\right]-\left[\frac{\partial T_{n}}{\partial x_{1}}, \frac{\partial P_{0}}{\partial x_{1}}\right]\right\}=0, \\
& \frac{\partial}{\partial x_{2}}\left\{\operatorname{tr}\left(F_{n+1,1} P_{0}\right)\right\}-\frac{\partial}{\partial x_{1}}\left\{\operatorname{tr}\left(F_{n+1,2} P_{0}\right)\right\} \\
& =\operatorname{tr}\left(\frac{\partial F_{n+1,1}}{\partial x_{2}} P_{0}-\frac{\partial F_{n+1,2}}{\partial x_{1}} P_{0}+F_{n+1,1} \frac{\partial P_{0}}{\partial x_{2}}-F_{n+1,2} \frac{\partial P_{0}}{\partial x_{1}}\right) \\
& =\operatorname{tr}\left[\left\{\frac{\partial^{2} T_{n}}{\partial x_{2} \partial x_{1}}-\frac{\partial}{\partial x_{2}}\left(\sum_{i=0}^{n-1} P_{n+1-i} T_{i}\right)\right\} P_{0}\right. \\
& -\left\{\frac{\partial^{2} T_{n}}{\partial x_{1} \partial x_{2}}-\frac{\partial}{\partial x_{1}}\left(\sum_{i=0}^{n-1} Q_{n+1-i} T_{i}\right)+\left[\frac{\partial T_{n}}{\partial x_{1}}, \frac{\partial P_{0}}{\partial x_{1}}\right]\right\} P_{0} \\
& \left.+2 F_{n+1,1} \frac{\partial P_{0}}{\partial x_{1}} P_{0}-\left(P_{0} F_{n+1,1}+F_{n+1,1} P_{0}\right) \frac{\partial P_{0}}{\partial x_{1}}\right] \\
& =\operatorname{tr}\left\{\frac{\partial P_{0}}{\partial x_{1}}\left[P_{0}, \sum_{i=0}^{n-1} P_{n+1-i} T_{i}\right]-\left[\frac{\partial T_{n}}{\partial x_{1}}, \frac{\partial P_{0}}{\partial x_{1}}\right] P_{0}+\left[P_{0}, F_{n+1,1}\right] \frac{\partial P_{0}}{\partial x_{1}}\right\} \\
& =\operatorname{tr}\left\{\frac{\partial P_{0}}{\partial x_{1}}\left[P_{0}, \frac{\partial T_{n}}{\partial x_{1}}\right]-\left[\frac{\partial T_{n}}{\partial x_{1}}, \frac{\partial P_{0}}{\partial x_{1}}\right] P_{0}\right\}=\operatorname{tr}\left[\frac{\partial P_{0}}{\partial x_{1}} P_{0}, \frac{\partial T_{n}}{\partial x_{1}}\right]=0,
\end{aligned}
$$

$\frac{\partial}{\partial x_{2}}\left\{\operatorname{tr}\left(F_{n+1,1} P_{0}^{2}\right)\right\}-\frac{\partial}{\partial x_{1}}\left\{\operatorname{tr}\left(F_{n+1,2} P_{0}^{2}\right)\right\}$

$$
\begin{aligned}
= & \operatorname{tr}\left(\frac{\partial F_{n+1,1}}{\partial x_{2}} P_{0}^{2}-\frac{\partial F_{n+1,2}}{\partial x_{1}} P_{0}^{2}+F_{n+1,1} \frac{\partial}{\partial x_{2}}\left(P_{0}^{2}\right)-F_{n+1,2} \frac{\partial}{\partial x_{1}}\left(P_{0}^{2}\right)\right) \\
= & \operatorname{tr}\left[\left\{\frac{\partial^{2} T_{n}}{\partial x_{2} \partial x_{1}}-\frac{\partial}{\partial x_{2}}\left(\sum_{i=0}^{n-1} P_{n+1-i} T_{i}\right)\right\} P_{0}^{2}\right. \\
& -\left\{\frac{\partial^{2} T_{n}}{\partial x_{1} \partial x_{2}}-\frac{\partial}{\partial x_{1}}\left(\sum_{i=0}^{n-1} Q_{n+1-i} T_{i}\right)+\left[\frac{\partial T_{n}}{\partial x_{1}}, \frac{\partial P_{0}}{\partial x_{1}}\right]\right\} P_{0}^{2} \\
& +F_{n+1,1}\left(2 P_{0} \frac{\partial P_{0}}{\partial x_{1}} P_{0}+2 \frac{\partial P_{0}}{\partial x_{1}} P_{0}^{2}\right) \\
& \left.-\left(P_{0} F_{n+1,1}+F_{n+1,1} P_{0}\right)\left(\frac{\partial P_{0}}{\partial x_{1}} P_{0}+P_{0} \frac{\partial P_{0}}{\partial x_{1}}\right)\right]
\end{aligned}
$$




$$
\begin{aligned}
& =\operatorname{tr}\left\{\frac{\partial P_{0}}{\partial x_{1}}\left[P_{0}^{2}, \sum_{i=0}^{n-1} P_{n+1-i} T_{i}\right]-\left[\frac{\partial T_{n}}{\partial x_{1}}, \frac{\partial P_{0}}{\partial x_{1}}\right] P_{0}^{2}+\left[P_{0}^{2}, F_{n+1,1}\right] \frac{\partial P_{0}}{\partial x_{1}}\right\} \\
& =\operatorname{tr}\left\{\frac{\partial P_{0}}{\partial x_{1}}\left[P_{0}^{2}, \frac{\partial T_{n}}{\partial x_{1}}\right]-\left[\frac{\partial T_{n}}{\partial x_{1}}, \frac{\partial P_{0}}{\partial x_{1}}\right] P_{0}^{2}\right\}=\operatorname{tr}\left[\frac{\partial P_{0}}{\partial x_{1}} P_{0}^{2}, \frac{\partial T_{n}}{\partial x_{1}}\right]=0 .
\end{aligned}
$$

Thus we obtain (4.91).

The system (4.86) means that $f_{n, k}(x) \quad(k=0,1,2)$ vanishes on $\left\{\left(x_{1}, 0\right) ; x_{1} \neq 0\right\}$. Thus, by Lemmas 4.21 and 4.22 and the holomorphy of $f_{n, k}(x)$, we find that

$$
\left.\left(\begin{array}{l}
f_{n, 0} \\
f_{n, 1} \\
f_{n, 2}
\end{array}\right)\right|_{x_{2}=0}=0
$$

Hence, by the uniqueness of solutions for the initial value problem (4.91) and (4.92), we obtain (4.90).

We have thus obtained a holomorphic solution of (4.81) near $x=0$, that is, we have constructed $T_{n}(x)$ satisfying (4.79).

The solution $\left(c_{n, 0}(x), c_{n, 1}(x), c_{n, 2}(x)\right)$ of (4.81) thus obtained enjoys the following property:

Lemma 4.25. The solution $\left(c_{n, 0}(x), c_{n, 1}(x), c_{n, 2}(x)\right)$ of the system $(4.81)$ is holomorphic in $D_{\rho}$ and has the integral representation

$$
\left\{\begin{array}{l}
c_{n, 0}(x)=\frac{1}{3} \int_{0}^{1}\left\{-x_{1} h_{n, 0}\left(x_{1} s, x_{2} s^{2 / 3}\right)+2 x_{2} s^{-1 / 3} g_{n, 0}\left(x_{1} s, x_{2} s^{2 / 3}\right)\right\} d s \\
c_{n, 1}(x)=\frac{1}{3} \int_{0}^{1}\left\{4 s^{-2 / 3} h_{n, 1}\left(x_{1} s, x_{2} s^{2 / 3}\right)+2 x_{2} g_{n, 1}\left(x_{1} s, x_{2} s^{2 / 3}\right)\right\} d s \\
c_{n, 2}(x)=\frac{1}{3} \int_{0}^{1}\left\{4 s^{-1 / 3} h_{n, 2}\left(x_{1} s, x_{2} s^{2 / 3}\right)+2 x_{2} s^{1 / 3} g_{n, 2}\left(x_{1} s, x_{2} s^{2 / 3}\right)\right\} d s .
\end{array}\right.
$$

Proof. Using (4.81), we find that $\left(c_{n, 0}(x), c_{n, 1}(x), c_{n, 2}(x)\right)$ satisfies

$$
\left\{3 x_{1} \frac{\partial}{\partial x_{1}}+2 x_{2} \frac{\partial}{\partial x_{2}}+\operatorname{diag}(0,1,2)\right\}\left(\begin{array}{c}
c_{n, 0} \\
c_{n, 1} \\
c_{n, 2}
\end{array}\right)=\left(\begin{array}{c}
-x_{1} h_{n, 0}(x)+2 x_{2} g_{n, 0}(x) \\
4 h_{n, 1}(x)+2 x_{2} g_{n, 1}(x) \\
4 h_{n, 2}(x)+2 x_{2} g_{n, 2}(x)
\end{array}\right) .
$$

Since a unique holomorphic solution of this system near $x=0$ with $c_{n, 0}(0)=0$ is represented by the right-hand side of (4.93), we obtain the integral representation.

Furthermore, by (4.93) and the holomorphy of $g_{n, k}(x), h_{n, k}(x)$ in $D_{\rho}$, we also find that $\left(c_{n, 0}(x), c_{n, 1}(x), c_{n, 2}(x)\right)$ is holomorphic in $D_{\rho}$. 
This completes the proof of the assertion $\left(\mathcal{S}_{n}\right)$. In other words, our induction on $n$ is now completed.

\section{$\S 5$. Estimate of the transformation}

In this section we prove the estimate (4.74) of the transformation constructed in Subsection 4.3.

Before proving the estimate, we prepare the following

Lemma 5.1. Let $A(x)$ be a $3 \times 3$ matrix with holomorphic entries in $D_{\rho}$. Suppose that there exist a positive constant $C$ and an integer $n \geq 1$ such that for any $\rho / 2<\sigma<\rho$,

$$
\|A\|_{\sigma, \sigma} \leq C n !(\rho-\sigma)^{-n}
$$

Then, for any $\rho / 2<\sigma<\rho$,

$$
\left\|\frac{\partial A}{\partial x_{j}}\right\|_{\sigma, \sigma} \leq e C(n+1) !(\rho-\sigma)^{-n-1} \quad(j=1,2) .
$$

Proof. Since the proof is similar for $j=1,2$, we show (5.2) only for $j=1$. For $\left|x_{1}\right|,\left|x_{2}\right| \leq \sigma$ we have

$$
\begin{aligned}
\frac{\partial A}{\partial x_{1}}(x) & =\frac{1}{2 \pi \sqrt{-1}} \int_{\left|t-x_{1}\right|=\frac{\rho-\sigma}{n+1}} \frac{A\left(t, x_{2}\right)}{\left(t-x_{1}\right)^{2}} d t \\
& =\frac{n+1}{2 \pi(\rho-\sigma)} \int_{0}^{2 \pi} A\left(x_{1}+\frac{\rho-\sigma}{n+1} e^{\sqrt{-1} \theta}, x_{2}\right) e^{-\sqrt{-1} \theta} d \theta .
\end{aligned}
$$

Hence

$$
\begin{aligned}
\left\|\frac{\partial A}{\partial x_{1}}\right\|_{\sigma, \sigma} & \leq \frac{n+1}{2 \pi(\rho-\sigma)} \int_{0}^{2 \pi}\|A\|_{\frac{n \sigma+\rho}{n+1}, \sigma} d \theta \leq \frac{n+1}{\rho-\sigma}\|A\|_{\frac{n \sigma+\rho}{n+1}, \frac{n \sigma+\rho}{n+1}} \\
& \leq\left(1-\frac{1}{n+1}\right)^{-n} C(n+1) !(\rho-\sigma)^{-n-1} \leq e C(n+1) !(\rho-\sigma)^{-n-1} .
\end{aligned}
$$

Combining (4.73) and Lemma 5.1, we can prove

Lemma 5.2. Replacing $C$ and $\alpha$ in (4.73) by new ones, we have the following estimates for any $\rho / 2<\sigma<\rho$ :

$$
\begin{aligned}
& \left\|P_{0}\right\|_{\sigma, \sigma},\left\|\frac{\partial P_{0}}{\partial x_{j}}\right\|_{\sigma, \sigma},\left\|Q_{0}\right\|_{\sigma, \sigma},\left\|\frac{\partial Q_{0}}{\partial x_{j}}\right\|_{\sigma, \sigma} \leq C, \\
& \left\|P_{1}\right\|_{\sigma, \sigma},\left\|\frac{\partial P_{1}}{\partial x_{j}}\right\|_{\sigma, \sigma},\left\|Q_{1}\right\|_{\sigma, \sigma},\left\|\frac{\partial Q_{1}}{\partial x_{j}}\right\|_{\sigma, \sigma} \leq C(\rho-\sigma)^{-1},
\end{aligned}
$$




$$
\begin{aligned}
\left\|P_{n}\right\|_{\sigma, \sigma},\left\|\frac{\partial P_{n}}{\partial x_{j}}\right\|_{\sigma, \sigma},\left\|Q_{n}\right\|_{\sigma, \sigma},\left\|\frac{\partial Q_{n}}{\partial x_{j}}\right\|_{\sigma, \sigma} & \\
\leq & C \alpha^{n-2}(n-1) !(\rho-\sigma)^{-n+1} \quad(n \geq 2) .
\end{aligned}
$$

Proof. Since $n \leq 2^{n}$ and $(\rho-\sigma)^{n-1} \leq \rho^{n-1}$ for any $n \geq 2$, we have

$$
\begin{aligned}
\left\|P_{n}\right\|_{\sigma, \sigma} & \leq C \alpha^{n} n ! \leq C \alpha^{2} \alpha^{n-2} n(n-1) !(\rho-\sigma)^{n-1}(\rho-\sigma)^{-n+1} \\
& \leq 4 C \alpha^{2} \rho(2 \alpha \rho)^{n-2}(n-1) !(\rho-\sigma)^{-n+1} .
\end{aligned}
$$

Similarly, since $n(n-1) \leq 4^{n}$ and $(\rho-\sigma)^{n-2} \leq \rho^{n-2}$ for any $n \geq 2$, we also have

$$
\begin{aligned}
\left\|P_{n}\right\|_{\sigma, \sigma} & \leq C \alpha^{n} n ! \leq C \alpha^{2} \alpha^{n-2} n(n-1)(n-2) !(\rho-\sigma)^{n-2}(\rho-\sigma)^{-n+2} \\
& \leq 16 C \alpha^{2}(4 \alpha \rho)^{n-2}(n-2) !(\rho-\sigma)^{-n+2}
\end{aligned}
$$

Hence, by Lemma 5.1, we obtain

$$
\left\|\frac{\partial P_{n}}{\partial x_{j}}\right\|_{\sigma, \sigma} \leq e 16 C \alpha^{2}(4 \alpha \rho)^{n-2}(n-1) !(\rho-\sigma)^{-n+1} .
$$

The estimates for $Q_{n}(x)$ and $\left(\partial Q_{n} / \partial x_{j}\right)(x)$ can be obtained in a similar way.

Furthermore, we may assume that

$$
\left\|T_{1}\right\|_{\sigma, \sigma},\left\|\frac{\partial T_{1}}{\partial x_{j}}\right\|_{\sigma, \sigma} \leq C(\rho-\sigma)^{-1} .
$$

Note that, since $T_{0}(x)=I$, we also have

$$
\left\|T_{0}\right\|_{\sigma, \sigma}=3, \quad\left\|\frac{\partial T_{0}}{\partial x_{j}}\right\|_{\sigma, \sigma}=0 .
$$

Making use of these lemmas, we prove the estimate (4.74) for $\left\{T_{n}(x)\right\}_{n \geq 0}$. In what follows, we may assume (by changing $\rho$ if necessary) $0<\rho<27 / 8$.

Proposition 5.3. There exist some positive constants $\widetilde{C} \geq C$ and $\widetilde{\alpha} \geq \alpha$ such that for any $n \geq 2$ and $\rho / 2<\sigma<\rho$,

$$
\left\|T_{n}\right\|_{\sigma, \sigma},\left\|\frac{\partial T_{n}}{\partial x_{j}}\right\|_{\sigma, \sigma} \leq \widetilde{C} \widetilde{\alpha}^{n-2} n !(\rho-\sigma)^{-n} .
$$

We prove Proposition 5.3 by induction on $n$. We assume that (5.8) holds up to $n-1$, that is, for any $i=2, \ldots, n-1$ and $\rho / 2<\sigma<\rho$ we have

$$
\left\|T_{i}\right\|_{\sigma, \sigma},\left\|\frac{\partial T_{i}}{\partial x_{j}}\right\|_{\sigma, \sigma} \leq \widetilde{C} \widetilde{\alpha}^{i-2} i !(\rho-\sigma)^{-i} .
$$


Under these assumptions we prove that, for any $\rho / 2<\sigma<\rho$,

$$
\left\|T_{n}\right\|_{\sigma, \sigma},\left\|\frac{\partial T_{n}}{\partial x_{j}}\right\|_{\sigma, \sigma} \leq \widetilde{C} \widetilde{\alpha}^{n-2} n !(\rho-\sigma)^{-n}
$$

(by choosing $\widetilde{C}$ and $\widetilde{\alpha}$ suitably).

We first show

Lemma 5.4. For any $\rho / 2<\sigma<\rho$, we have

$$
\begin{gathered}
\left\|T_{n, 2}\right\|_{\sigma, \sigma} \leq(2+C)\left(\widetilde{C} \widetilde{\alpha}^{-1}+C \widetilde{C} \widetilde{\alpha}^{-2}+C^{2} \widetilde{\alpha}^{-1}+C\right) \widetilde{\alpha}^{n-2}(n-1) !(\rho-\sigma)^{-n+1}, \\
(5.12)\left\|\frac{\partial T_{n, 2}}{\partial x_{j}}\right\|_{\sigma, \sigma} \leq e(2+C)\left(\widetilde{C} \widetilde{\alpha}^{-1}+C \widetilde{C} \widetilde{\alpha}^{-2}+C^{2} \widetilde{\alpha}^{-1}+C\right) \widetilde{\alpha}^{n-2} n !(\rho-\sigma)^{-n} .
\end{gathered}
$$

Proof. First, we estimate $F_{n, 1}$ :

$$
\begin{aligned}
& \left\|F_{n, 1}\right\|_{\sigma, \sigma}=\left\|\frac{\partial T_{n-1}}{\partial x_{1}}-\sum_{i=0}^{n-2} P_{n-i} T_{i}\right\|_{\sigma, \sigma} \\
& \leq\left\|\frac{\partial T_{n-1}}{\partial x_{1}}\right\|_{\sigma, \sigma}+\sum_{i=2}^{n-2}\left\|P_{n-i}\right\|_{\sigma, \sigma}\left\|T_{i}\right\|_{\sigma, \sigma}+\left\|P_{n-1}\right\|_{\sigma, \sigma}\left\|T_{1}\right\|_{\sigma, \sigma}+\left\|P_{n}\right\|_{\sigma, \sigma} \\
& \leq \widetilde{C} \widetilde{\alpha}^{n-3}(n-1) !(\rho-\sigma)^{-n+1} \\
& \quad+\sum_{i=2}^{n-2}\left\{C \alpha^{n-i-2}(n-i-1) !(\rho-\sigma)^{-n+i+1}\right\} \times\left\{\widetilde{C} \widetilde{\alpha}^{i-2} i !(\rho-\sigma)^{-i}\right\} \\
& \quad+\left\{C \alpha^{n-3}(n-2) !(\rho-\sigma)^{-n+2}\right\} \times\left\{C(\rho-\sigma)^{-1}\right\}+C \alpha^{n-2}(n-1) !(\rho-\sigma)^{-n+1} \\
& \leq \widetilde{C} \widetilde{\alpha}^{n-3}(n-1) !(\rho-\sigma)^{-n+1}+C \widetilde{C} \widetilde{\alpha}^{n-4}(\rho-\sigma)^{-n+1} \sum_{i=2}^{n-2}(n-i-1) ! i ! \\
& \quad+C^{2} \widetilde{\alpha}^{n-3}(n-2) !(\rho-\sigma)^{-n+1}+C \widetilde{\alpha} \widetilde{\alpha}^{n-2}(n-1) !(\rho-\sigma)^{-n+1} \\
& \leq\left(\widetilde{C} \widetilde{\alpha}^{-1}+C \widetilde{C} \widetilde{\alpha}^{-2}+C^{2} \widetilde{\alpha}^{-1}+C\right) \widetilde{\alpha}^{n-2}(n-1) !(\rho-\sigma)^{-n+1} .
\end{aligned}
$$

Hence we get

$$
\begin{aligned}
& \left\|T_{n, 2}\right\|_{\sigma, \sigma}=\left\|\Lambda F_{n, 1}+\Lambda^{2} F_{n, 1} P_{0}\right\|_{\sigma, \sigma} \\
& \quad \leq\|\Lambda\|_{\sigma, \sigma}\left\|F_{n, 1}\right\|_{\sigma, \sigma}+\left\|\Lambda^{2}\right\|_{\sigma, \sigma}\left\|F_{n, 1}\right\|_{\sigma, \sigma}\left\|P_{0}\right\|_{\sigma, \sigma} \leq(2+C)\left\|F_{n, 1}\right\|_{\sigma, \sigma} \\
& \quad \leq(2+C)\left(\widetilde{C} \widetilde{\alpha}^{-1}+C \widetilde{C} \widetilde{\alpha}^{-2}+C^{2} \widetilde{\alpha}^{-1}+C\right) \widetilde{\alpha}^{n-2}(n-1) !(\rho-\sigma)^{-n+1} .
\end{aligned}
$$

The estimate (5.12) can be obtained by (5.13) and Lemma 5.1. 
Next, we estimate $T_{n, 1}(x)$ and $\left(\partial T_{n, 1} / \partial x_{j}\right)(x)$. For this purpose, we first prove

Lemma 5.5. Let $C_{1}$ be the positive constant defined by

$$
\begin{aligned}
& C_{1}=\max \left\{1+2 C, C^{2}\right\} \\
& \times\left\{(e+2 C \rho)(2+C)\left(\widetilde{C} \widetilde{\alpha}^{-1}+C \widetilde{C} \widetilde{\alpha}^{-2}+C^{2} \widetilde{\alpha}^{-1}+C\right)+C \widetilde{C} \widetilde{\alpha}^{-1}+C^{2}+C \alpha\right\} .
\end{aligned}
$$

Then

$$
\left|g_{n, k}\right|_{\sigma, \sigma},\left|h_{n, k}\right|_{\sigma, \sigma} \leq C_{1} \widetilde{\alpha}^{n-2} n !(\rho-\sigma)^{-n}
$$

for any $k=0,1,2$ and $\rho / 2<\sigma<\rho$.

Proof. We first estimate $F_{n+1,1}^{\dagger}$ and $F_{n+1,2}^{\dagger}$ :

$$
\begin{aligned}
\left\|F_{n+1,1}^{\dagger}\right\|_{\sigma, \sigma}= & \left\|\frac{\partial T_{n, 2}}{\partial x_{1}}-\sum_{i=0}^{n-1} P_{n+1-i} T_{i}\right\|_{\sigma, \sigma} \\
\leq & \left\|\frac{\partial T_{n, 2}}{\partial x_{1}}\right\|_{\sigma, \sigma}+\sum_{i=2}^{n-1}\left\|P_{n+1-i}\right\|_{\sigma, \sigma}\left\|T_{i}\right\|_{\sigma, \sigma}+\left\|P_{n}\right\|_{\sigma, \sigma}\left\|T_{1}\right\|_{\sigma, \sigma}+\left\|P_{n+1}\right\|_{\sigma, \sigma} \\
\leq & e(2+C)\left(\widetilde{C} \widetilde{\alpha}^{-1}+C \widetilde{C} \widetilde{\alpha}^{-2}+C^{2} \widetilde{\alpha}^{-1}+C\right) \widetilde{\alpha}^{n-2} n !(\rho-\sigma)^{-n} \\
& +\sum_{i=2}^{n-2}\left\{C \alpha^{n-i-1}(n-i) !(\rho-\sigma)^{-n+i}\right\} \times\left\{\widetilde{C} \widetilde{\alpha}^{i-2} i !(\rho-\sigma)^{-i}\right\} \\
& +\left\{C \alpha^{n-2}(n-1) !(\rho-\sigma)^{-n+1}\right\} \times\left\{C(\rho-\sigma)^{-1}\right\}+C \alpha^{n-1} n !(\rho-\sigma)^{-n} \\
\leq & e(2+C)\left(\widetilde{C} \widetilde{\alpha}^{-1}+C \widetilde{C} \widetilde{\alpha}^{-2}+C^{2} \widetilde{\alpha}^{-1}+C\right) \widetilde{\alpha}^{n-2} n !(\rho-\sigma)^{-n} \\
& +C \widetilde{C} \widetilde{\alpha}^{n-3}(\rho-\sigma)^{-n} \sum_{i=2}^{n-1}(n-i) ! i !+C^{2} \widetilde{\alpha}^{n-2}(n-1) !(\rho-\sigma)^{-n} \\
& +C \widetilde{\alpha}^{n-2} \alpha n !(\rho-\sigma)^{-n} \\
\leq & \left\{e(2+C)\left(\widetilde{C} \widetilde{\alpha}^{-1}+C \widetilde{C} \widetilde{\alpha}^{-2}+C^{2} \widetilde{\alpha}^{-1}+C\right)+C \widetilde{C} \widetilde{\alpha}^{-1}+C^{2}+C \alpha\right\} \\
& \times \widetilde{\alpha}^{n-2} n !(\rho-\sigma)^{-n}, \\
\left\|F_{n+1,2}^{\dagger}\right\|_{\sigma, \sigma} & =\left\|\frac{\partial T_{n, 2}}{\partial x_{2}}+\left[T_{n, 2}, \frac{\partial P_{0}}{\partial x_{1}}\right]-\sum_{i=0}^{n-1} Q_{n+1-i} T_{i}\right\|_{\sigma, \sigma} \\
& \leq\left\|\frac{\partial T_{n, 2}}{\partial x_{2}}\right\|\left\|_{\sigma, \sigma}+2\right\| T_{n, 2}\left\|_{\sigma, \sigma}\right\| \frac{\partial P_{0}}{\partial x_{1}} \|_{\sigma, \sigma} \\
& +\sum_{i=2}^{n-1}\left\|Q_{n+1-i}\right\|_{\sigma, \sigma}\left\|T_{i}\right\|_{\sigma, \sigma}+\left\|Q_{n}\right\|_{\sigma, \sigma}\left\|T_{1}\right\|_{\sigma, \sigma}+\left\|Q_{n+1}\right\|_{\sigma, \sigma}
\end{aligned}
$$




$$
\begin{aligned}
\leq & e(2+C)\left(\widetilde{C} \widetilde{\alpha}^{-1}+C \widetilde{C} \widetilde{\alpha}^{-2}+C^{2} \widetilde{\alpha}^{-1}+C\right) \widetilde{\alpha}^{n-2} n !(\rho-\sigma)^{-n} \\
& +2 C(2+C)\left(\widetilde{C} \widetilde{\alpha}^{-1}+C \widetilde{C} \widetilde{\alpha}^{-2}+C^{2} \widetilde{\alpha}^{-1}+C\right) \widetilde{\alpha}^{n-2}(n-1) !(\rho-\sigma)^{-n+1} \\
& +\sum_{i=2}^{n-2}\left\{C \alpha^{n-i-1}(n-i) !(\rho-\sigma)^{-n+i}\right\} \times\left\{\widetilde{C} \widetilde{\alpha}^{i-2} i !(\rho-\sigma)^{-i}\right\} \\
& +\left\{C \alpha^{n-2}(n-1) !(\rho-\sigma)^{-n+1}\right\} \times\left\{C(\rho-\sigma)^{-1}\right\}+C \alpha^{n-1} n !(\rho-\sigma)^{-n} \\
\leq & \left(e+2 C \frac{\rho-\sigma}{n}\right)(2+C)\left(\widetilde{C} \widetilde{\alpha}^{-1}+C \widetilde{C} \widetilde{\alpha}^{-2}+C^{2} \widetilde{\alpha}^{-1}+C\right) \widetilde{\alpha}^{n-2} n !(\rho-\sigma)^{-n} \\
& +C \widetilde{C} \widetilde{\alpha}^{n-3}(\rho-\sigma)^{-n} \sum_{i=2}^{n-1}(n-i) ! i !+C^{2} \widetilde{\alpha}^{n-2}(n-1) !(\rho-\sigma)^{-n} \\
& +C \widetilde{\alpha}^{n-2} \alpha n !(\rho-\sigma)^{-n} \\
\leq & \left\{(e+2 C \rho)(2+C)\left(\widetilde{C} \widetilde{\alpha}^{-1}+C \widetilde{C} \widetilde{\alpha}^{-2}+C^{2} \widetilde{\alpha}^{-1}+C\right)+C \widetilde{C} \widetilde{\alpha}^{-1}+C^{2}+C \alpha\right\} \\
& \times \widetilde{\alpha}^{n-2} n !(\rho-\sigma)^{-n} .
\end{aligned}
$$

Hence, if we define

$$
C_{1}^{\dagger}=(e+2 C \rho)(2+C)\left(\widetilde{C} \widetilde{\alpha}^{-1}+C \widetilde{C} \widetilde{\alpha}^{-2}+C^{2} \widetilde{\alpha}^{-1}+C\right)+C \widetilde{C} \widetilde{\alpha}^{-1}+C^{2}+C \alpha,
$$

we obtain

$$
\left\|F_{n+1,1}^{\dagger}\right\|_{\sigma, \sigma},\left\|F_{n+1,2}^{\dagger}\right\|_{\sigma, \sigma} \leq C_{1}^{\dagger} \widetilde{\alpha}^{n-2} n !(\rho-\sigma)^{-n} .
$$

Using these estimates, we now prove the estimates (5.15). Since the $(1,1),(1,2)$ and $(1,3)$ entries of $g_{n, 2}(x) P_{0}^{2}+g_{n, 1}(x) P_{0}+g_{n, 0}(x)$ are $g_{n, 0}(x), g_{n, 1}(x), g_{n, 2}(x)$, respectively, we get

$$
\begin{aligned}
\left|g_{n, k}\right|_{\sigma, \sigma} & \leq\left\|g_{n, 2} P_{0}^{2}+g_{n, 1} P_{0}+g_{n, 0}\right\|_{\sigma, \sigma} \leq\left\|-F_{n+1,2}^{\dagger}+F_{n+1,1}^{\dagger} P_{0}+P_{0} F_{n+1,1}^{\dagger}\right\|_{\sigma, \sigma} \\
& \leq\left\|F_{n+1,2}^{\dagger}\right\|_{\sigma, \sigma}+2\left\|F_{n+1,1}^{\dagger}\right\|_{\sigma, \sigma}\left\|P_{0}\right\|_{\sigma, \sigma} \leq(1+2 C) C_{1}^{\dagger} \widetilde{\alpha}^{n-2} n !(\rho-\sigma)^{-n} .
\end{aligned}
$$

Furthermore,

$$
\begin{aligned}
\left|h_{n, 0}\right|_{\sigma, \sigma} & \leq\left|\operatorname{tr}\left(F_{n+1,1}^{\dagger}\right)\right|_{\sigma, \sigma} \leq\left\|F_{n+1,1}^{\dagger}\right\|_{\sigma, \sigma} \leq C_{1}^{\dagger} \widetilde{\alpha}^{n-2} n !(\rho-\sigma)^{-n}, \\
\left|h_{n, 1}\right|_{\sigma, \sigma} & \leq\left|\operatorname{tr}\left(F_{n+1,1}^{\dagger} P_{0}^{2}\right)\right|_{\sigma, \sigma} \leq\left\|F_{n+1,1}^{\dagger} P_{0}^{2}\right\|_{\sigma, \sigma} \\
& \leq\left\|F_{n+1,1}^{\dagger}\right\|_{\sigma, \sigma}\left\|P_{0}\right\|_{\sigma, \sigma}^{2} \leq C^{2} C_{1}^{\dagger} \widetilde{\alpha}^{n-2} n !(\rho-\sigma)^{-n}, \\
\left|h_{n, 2}\right|_{\sigma, \sigma} & \leq\left|\operatorname{tr}\left(F_{n+1,1}^{\dagger} P_{0}\right)\right|_{\sigma, \sigma} \leq\left\|F_{n+1,1}^{\dagger} P_{0}\right\|_{\sigma, \sigma} \\
& \leq\left\|F_{n+1,1}^{\dagger}\right\|_{\sigma, \sigma}\left\|P_{0}\right\|_{\sigma, \sigma} \leq C C_{1}^{\dagger} \widetilde{\alpha}^{n-2} n !(\rho-\sigma)^{-n} .
\end{aligned}
$$

Thus the estimates (5.15) immediately follow if we define $C_{1}$ by (5.14).

We next estimate $c_{n, k}(x)(k=0,1,2)$. 
Lemma 5.6. Let $C_{2}$ be the positive constant defined by

$$
\max \left\{4, \frac{1000}{27 \rho-8 \rho^{2}}, \frac{1000}{27(\rho / 2)-8(\rho / 2)^{2}}, \frac{6000 C}{27 \rho-8 \rho^{2}}+1, \frac{6000 C}{27(\rho / 2)-8(\rho / 2)^{2}}+1\right\} .
$$

Then

$$
\left|c_{n, k}\right|_{\sigma, \sigma},\left|\frac{\partial c_{n, k}}{\partial x_{j}}\right|_{\sigma, \sigma} \leq C_{2} \sum_{i=0}^{2}\left(\left|g_{n, i}\right|_{\sigma, \sigma}+\left|h_{n, i}\right|_{\sigma, \sigma}\right)
$$

for any $k=0,1,2$ and $\rho / 2<\sigma<\rho$.

In the proof of Lemma 5.6, we need the following two sublemmas.

Sublemma 5.7. For any $\rho / 2<\sigma<\rho, c_{n, k}(x)(k=0,1,2)$ satisfy

$$
\begin{aligned}
\left|c_{n, 0}\right|_{\sigma, \sigma} & \leq \frac{\sigma}{3}\left|h_{n, 0}\right|_{\sigma, \sigma}+\sigma\left|g_{n, 0}\right|_{\sigma, \sigma}, \\
\left|c_{n, 1}\right|_{\sigma, \sigma} & \leq 4\left|h_{n, 1}\right|_{\sigma, \sigma}+\frac{2 \sigma}{3}\left|g_{n, 1}\right|_{\sigma, \sigma}, \\
\left|c_{n, 2}\right|_{\sigma, \sigma} & \leq 2\left|h_{n, 2}\right|_{\sigma, \sigma}+\frac{\sigma}{2}\left|g_{n, 2}\right|_{\sigma, \sigma} .
\end{aligned}
$$

Proof. Thanks to the integral representations (4.93) for $c_{n, k}(x)$, we obtain the following estimates for $\left|x_{j}\right| \leq \sigma(j=1,2)$ :

$$
\begin{aligned}
\left|c_{n, 0}(x)\right| & =\left|\frac{1}{3} \int_{0}^{1}\left\{-x_{1} h_{n, 0}\left(x_{1} s, x_{2} s^{2 / 3}\right)+2 x_{2} s^{-1 / 3} g_{n, 0}\left(x_{1} s, x_{2} s^{2 / 3}\right)\right\} d s\right| \\
& \leq \frac{1}{3} \int_{0}^{1}\left\{\sigma\left|h_{n, 0}\left(x_{1} s, x_{2} s^{2 / 3}\right)\right|+2 \sigma s^{-1 / 3}\left|g_{n, 0}\left(x_{1} s, x_{2} s^{2 / 3}\right)\right|\right\} d s \\
& \leq \frac{1}{3} \int_{0}^{1}\left\{\sigma\left|h_{n, 0}\right|_{\sigma, \sigma}+2 \sigma s^{-1 / 3}\left|g_{n, 0}\right|_{\sigma, \sigma}\right\} d s \\
& =\frac{\sigma}{3}\left|h_{n, 0}\right|_{\sigma, \sigma}+\sigma\left|g_{n, 0}\right|_{\sigma, \sigma}, \\
\left|c_{n, 1}(x)\right| & =\left|\frac{1}{3} \int_{0}^{1}\left\{4 s^{-2 / 3} h_{n, 1}\left(x_{1} s, x_{2} s^{2 / 3}\right)+2 x_{2} g_{n, 1}\left(x_{1} s, x_{2} s^{2 / 3}\right)\right\} d s\right| \\
& \leq \frac{1}{3} \int_{0}^{1}\left\{4 s^{-2 / 3}\left|h_{n, 1}\left(x_{1} s, x_{2} s^{2 / 3}\right)\right|+2 \sigma\left|g_{n, 1}\left(x_{1} s, x_{2} s^{2 / 3}\right)\right|\right\} d s \\
& \leq \frac{1}{3} \int_{0}^{1}\left\{4 s^{-2 / 3}\left|h_{n, 1}\right|_{\sigma, \sigma}+2 \sigma\left|g_{n, 1}\right|_{\sigma, \sigma}\right\} d s \\
& =4\left|h_{n, 1}\right|_{\sigma, \sigma}+\frac{2 \sigma}{3}\left|g_{n, 1}\right|_{\sigma, \sigma},
\end{aligned}
$$




$$
\begin{aligned}
\left|c_{n, 2}(x)\right| & =\left|\frac{1}{3} \int_{0}^{1}\left\{4 s^{-1 / 3} h_{n, 2}\left(x_{1} s, x_{2} s^{2 / 3}\right)+2 x_{2} s^{1 / 3} g_{n, 2}\left(x_{1} s, x_{2} s^{2 / 3}\right)\right\} d s\right| \\
& \leq \frac{1}{3} \int_{0}^{1}\left\{4 s^{-1 / 3}\left|h_{n, 2}\left(x_{1} s, x_{2} s^{2 / 3}\right)\right|+2 \sigma s^{1 / 3}\left|g_{n, 2}\left(x_{1} s, x_{2} s^{2 / 3}\right)\right|\right\} d s \\
& \leq \frac{1}{3} \int_{0}^{1}\left\{4 s^{-1 / 3}\left|h_{n, 2}\right|_{\sigma, \sigma}+2 \sigma s^{1 / 3}\left|g_{n, 2}\right|_{\sigma, \sigma}\right\} d s \\
& =2\left|h_{n, 2}\right|_{\sigma, \sigma}+\frac{\sigma}{2}\left|g_{n, 2}\right|_{\sigma, \sigma} .
\end{aligned}
$$

Sublemma 5.8. Let $f(x)$ be a holomorphic function in $D_{\rho}$. For any $\rho / 2<\sigma<\rho$,

$$
\sup _{\left|x_{1}\right|,\left|x_{2}\right| \leq \sigma}|f(x)|=\sup _{\left|x_{1}\right|=\left|x_{2}\right|=\sigma}|f(x)| .
$$

Sublemma 5.8 is an immediate consequence of the maximum modulus principle for holomorphic functions.

Using these sublemmas, we prove Lemma 5.6.

Proof of Lemma 5.6. Let $R(x)$ be an adjugate matrix of $3 x_{1}+4 x_{2} P_{0}^{T}$, that is,

$$
R(x)=16 x_{2}^{2}\left(P_{0}^{T}\right)^{2}-12 x_{1} x_{2} P_{0}^{T}+9 x_{1}^{2}+8 x_{2}^{3}=\left(\begin{array}{ccc}
9 x_{1}^{2}+8 x_{2}^{3} & -4 x_{1} x_{2}^{2} & 3 x_{1}^{2} x_{2} \\
-12 x_{1} x_{2} & 9 x_{1}^{2} & 2 x_{1} x_{2}^{2} \\
16 x_{2}^{2} & -12 x_{1} x_{2} & 9 x_{1}^{2}
\end{array}\right) .
$$

Then

$$
R\left(3 x_{1}+4 x_{2} P_{0}^{T}\right)=\left(3 x_{1}+4 x_{2} P_{0}^{T}\right) R=x_{1}\left(27 x_{1}^{2}+8 x_{2}^{3}\right) .
$$

It follows from this relation and the first equation of (4.81) that

$$
\begin{aligned}
x_{1}\left(27 x_{1}^{2}+8 x_{2}^{3}\right) \frac{\partial}{\partial x_{1}}\left(\begin{array}{c}
c_{n, 0} \\
c_{n, 1} \\
c_{n, 2}
\end{array}\right) & =R\left(3 x_{1}+4 x_{2} P_{0}^{T}\right) \frac{\partial}{\partial x_{1}}\left(\begin{array}{c}
c_{n, 0} \\
c_{n, 1} \\
c_{n, 2}
\end{array}\right) \\
& =R\left(\begin{array}{c}
-x_{1} h_{n, 0} \\
-c_{n, 1}+4 h_{n, 1} \\
-2 c_{n, 2}+4 h_{n, 2}
\end{array}\right) .
\end{aligned}
$$

Furthermore, by the explicit form of $R(x)$,

$$
\|R\|_{\sigma, \sigma} \leq 67 \sigma^{2}+17 \sigma^{3} \text {. }
$$

Combining these properties of $R(x)$ with Sublemmas 5.7 and 5.8, we find

$$
\begin{aligned}
\left|\frac{\partial c_{n, k}}{\partial x_{1}}\right|_{\sigma, \sigma} & =\sup _{\left|x_{1}\right|=\left|x_{2}\right|=\sigma}\left|\frac{\partial c_{n, k}}{\partial x_{1}}(x)\right| \\
& =\sup _{\left|x_{1}\right|=\left|x_{2}\right|=\sigma}\left|\frac{1}{x_{1}\left(27 x_{1}^{2}+8 x_{2}^{3}\right)}\right| \cdot\left|x_{1}\left(27 x_{1}^{2}+8 x_{2}^{3}\right) \frac{\partial c_{n, k}}{\partial x_{1}}\right|_{\sigma, \sigma} \\
& \leq \frac{\|R\|_{\sigma, \sigma}}{27 \sigma^{3}-8 \sigma^{4}}\left(\left|x_{1} h_{n, 0}\right|_{\sigma, \sigma}+\left|-c_{n, 1}+4 h_{n, 1}\right|_{\sigma, \sigma}+\left|-2 c_{n, 2}+4 h_{n, 2}\right|_{\sigma, \sigma}\right)
\end{aligned}
$$




$$
\begin{aligned}
& \leq \frac{67+17 \sigma}{27 \sigma-8 \sigma^{2}}\left(\sigma\left|h_{n, 0}\right|_{\sigma, \sigma}+8\left|h_{n, 1}\right|_{\sigma, \sigma}+8\left|h_{n, 2}\right|_{\sigma, \sigma}+\frac{2 \sigma}{3}\left|g_{n, 1}\right|_{\sigma, \sigma}+\sigma\left|g_{n, 2}\right|_{\sigma, \sigma}\right) \\
& \leq \frac{1000}{27 \sigma-8 \sigma^{2}} \sum_{i=0}^{2}\left(\left|g_{n, i}\right|_{\sigma, \sigma}+\left|h_{n, i}\right|_{\sigma, \sigma}\right)
\end{aligned}
$$

in view of $\sigma<\rho<27 / 8$.

On the other hand, by the second equation of (4.81), we find

$$
\begin{aligned}
\left|\frac{\partial c_{n, k}}{\partial x_{2}}\right|_{\sigma, \sigma} & \leq 2\left\|P_{0}\right\|_{\sigma, \sigma} \sum_{i=0}^{2}\left|\frac{\partial c_{n, i}}{\partial x_{1}}\right|_{\sigma, \sigma}+\sum_{i=0}^{2}\left|g_{n, i}\right|_{\sigma, \sigma} \\
& \leq\left(\frac{6000 C}{27 \sigma-8 \sigma^{2}}+1\right) \sum_{i=0}^{2}\left(\left|g_{n, i}\right|_{\sigma, \sigma}+\left|h_{n, i}\right|_{\sigma, \sigma}\right) .
\end{aligned}
$$

Hence we obtain the estimates (5.17) for $C_{2}$ defined by (5.16).

Using Lemmas 5.5 and 5.6, we can estimate $T_{n, 1}(x)$ and $\left(\partial T_{n, 1} / \partial x_{j}\right)(x)$.

Lemma 5.9. For any $\rho / 2<\sigma<\rho$, we have

$$
\begin{aligned}
\left\|T_{n, 1}\right\|_{\sigma, \sigma} & \leq 6\left(C^{2}+C+3\right) C_{1} C_{2} \widetilde{\alpha}^{n-2} n !(\rho-\sigma)^{-n} \\
\left\|\frac{\partial T_{n, 1}}{\partial x_{j}}\right\|_{\sigma, \sigma} & \leq 6\left(3 C^{2}+2 C+3\right) C_{1} C_{2} \widetilde{\alpha}^{n-2} n !(\rho-\sigma)^{-n} .
\end{aligned}
$$

Proof. Using Lemmas 5.5 and 5.6, we find

$$
\begin{aligned}
\left\|T_{n, 1}\right\|_{\sigma, \sigma} \leq & \left\|c_{n, 2} P_{0}^{2}+c_{n, 1} P_{0}+c_{n, 0}\right\|_{\sigma, \sigma} \\
\leq & \left|c_{n, 2}\right|_{\sigma, \sigma}\left\|P_{0}\right\|_{\sigma, \sigma}^{2}+\left|c_{n, 1}\right|_{\sigma, \sigma}\left\|P_{0}\right\|_{\sigma, \sigma}+3\left|c_{n, 0}\right|_{\sigma, \sigma} \\
\leq & \left(C^{2}+C+3\right) C_{2} \sum_{i=0}^{2}\left(\left|g_{n, i}\right|_{\sigma, \sigma}+\left|h_{n, i}\right|_{\sigma, \sigma}\right) \\
\leq & 6\left(C^{2}+C+3\right) C_{1} C_{2} \widetilde{\alpha}^{n-2} n !(\rho-\sigma)^{-n}, \\
\left\|\frac{\partial T_{n, 1}}{\partial x_{j}}\right\|_{\sigma, \sigma} \leq & \left\|\frac{\partial c_{n, 2}}{\partial x_{j}} P_{0}^{2}+\frac{\partial c_{n, 1}}{\partial x_{j}} P_{0}+\frac{\partial c_{n, 0}}{\partial x_{j}}+c_{n, 2} \frac{\partial}{\partial x_{j}} P_{0}^{2}+c_{n, 1} \frac{\partial}{\partial x_{j}} P_{0}\right\|_{\sigma, \sigma} \\
\leq & \left|\frac{\partial c_{n, 2}}{\partial x_{j}}\right|_{\sigma, \sigma}\left\|P_{0}\right\|_{\sigma, \sigma}^{2}+\left|\frac{\partial c_{n, 1}}{\partial x_{j}}\right|_{\sigma, \sigma}\left\|P_{0}\right\|_{\sigma, \sigma}+3\left|\frac{\partial c_{n, 0}}{\partial x_{j}}\right|_{\sigma, \sigma} \\
& +2\left|c_{n, 2}\right|_{\sigma, \sigma}\left\|P_{0}\right\|_{\sigma, \sigma}\left\|\frac{\partial P_{0}}{\partial x_{j}}\right\|_{\sigma, \sigma}+\left|c_{n, 1}\right|_{\sigma, \sigma}\left\|\frac{\partial P_{0}}{\partial x_{j}}\right\|_{\sigma, \sigma} \\
\leq & \left(3 C^{2}+2 C+3\right) C_{2} \sum_{i=0}^{2}\left(\left|g_{n, i}\right|_{\sigma, \sigma}+\left|h_{n, i}\right|_{\sigma, \sigma}\right) \\
\leq & 6\left(3 C^{2}+2 C+3\right) C_{1} C_{2} \widetilde{\alpha}^{n-2} n !(\rho-\sigma)^{-n} .
\end{aligned}
$$


Using these lemmas, we now prove Proposition 5.3.

Proof of Proposition 5.3. By Lemmas 5.4 and 5.9, we obtain

$$
\begin{aligned}
\left\|T_{n}\right\|_{\sigma, \sigma} & \leq\left\|T_{n, 1}\right\|_{\sigma, \sigma}+\left\|T_{n, 2}\right\|_{\sigma, \sigma} \leq \widehat{C}_{1} \widetilde{\alpha}^{n-2} n !(\rho-\sigma)^{-n}, \\
\left\|\frac{\partial T_{n}}{\partial x_{j}}\right\|_{\sigma, \sigma} & \leq\left\|\frac{\partial T_{n, 1}}{\partial x_{j}}\right\|_{\sigma, \sigma}+\left\|\frac{\partial T_{n, 2}}{\partial x_{j}}\right\|_{\sigma, \sigma} \leq \widehat{C}_{2} \widetilde{\alpha}^{n-2} n !(\rho-\sigma)^{-n},
\end{aligned}
$$

where

$$
\begin{array}{r}
\widehat{C}_{1}=\left(\frac{\widehat{C}_{1,1}}{\widetilde{\alpha}}+\frac{\widehat{C}_{1,2}}{\widetilde{\alpha}^{2}}\right) \widetilde{C}+\left(\widehat{C}_{1,3}+\frac{\widehat{C}_{1,4}}{\widetilde{\alpha}}\right), \\
\widehat{C}_{2}=\left(\frac{\widehat{C}_{2,1}}{\widetilde{\alpha}}+\frac{\widehat{C}_{2,2}}{\widetilde{\alpha}^{2}}\right) \widetilde{C}+\left(\widehat{C}_{2,3}+\frac{\widehat{C}_{2,4}}{\widetilde{\alpha}}\right)
\end{array}
$$

with some positive constants $\widehat{C}_{i, j}(i=1,2, j=1,2,3,4)$ independent of $\widetilde{C}, \widetilde{\alpha}$, $\sigma, n$. Take a constant $\widetilde{\alpha}$ large enough so that

$$
\frac{\widehat{C}_{1,1}}{\widetilde{\alpha}}+\frac{\widehat{C}_{1,2}}{\widetilde{\alpha}^{2}}, \frac{\widehat{C}_{2,1}}{\widetilde{\alpha}}+\frac{\widehat{C}_{2,2}}{\widetilde{\alpha}^{2}}<1 .
$$

Then there exists a positive constant $\widetilde{C}$ satisfying $\widehat{C}_{1}, \widehat{C}_{2} \leq \widetilde{C}$. This completes the proof of Proposition 5.3.

Using Proposition 5.3, we can verify the estimate (4.74). In fact, taking $\sigma=$ $(3 / 4) \rho$, we obtain from $(5.8)$

$$
\left\|T_{n}\right\|_{(3 / 4) \rho,(3 / 4) \rho} \leq \widetilde{C} \widetilde{\alpha}^{n-2} n !(\rho / 4)^{-n}=\widetilde{C} \widetilde{\alpha}^{-2}(4 \widetilde{\alpha} / \rho)^{n} n ! .
$$

Hence, if we define positive constants $\widetilde{C}^{\dagger}, \widetilde{\alpha}^{\dagger}$ and $\rho^{\dagger}$ by

$$
\widetilde{C}^{\dagger}=\widetilde{C} \widetilde{\alpha}^{-2}, \quad \widetilde{\alpha}^{\dagger}=4 \widetilde{\alpha} / \rho, \quad \rho^{\dagger}=3 \rho / 4,
$$

the estimate (4.74) holds for $\widetilde{C}=\widetilde{C}^{\dagger}, \widetilde{\alpha}=\widetilde{\alpha}^{\dagger}$ and $\rho=\rho^{\dagger}$.

\section{Acknowledgements}

The author sincerely thanks Professor Yoshitsugu Takei, Professor Takahiro Kawai, Professor Tatsuya Koike and Dr. Shingo Kamimoto for their valuable advice and encouragement. The author also thanks Dr. Kazuo Kaneko for his comments on Theorem 4.4 .

\section{References}

[A] T. Aoki, Toward the exact WKB analysis for holonomic systems, RIMS Kôkyûroku 1433 (2005), 1-8 (in Japanese). 
S. Hirose

[AKT] T. Aoki, T. Kawai and Y. Takei, The Bender-Wu analysis and the Voros theory, in Special functions (Okayama, 1990), Springer, 1991, 1-29. Zbl 0782.35060 MR 1166808

[BNR] H. L. Berk, W. M. Nevins and K. V. Roberts, New Stokes' line in WKB theory, J. Math. Phys. 23 (1982), 988-1002. Zbl 0488.34050 MR 0659998

$[\mathrm{H}] \quad$ S. Hirose, On the Stokes geometry for the Pearcey system and the $(1,4)$ hypergeometric system, RIMS Kôkyûroku Bessatsu B40, 2013, 243-292.

[KO] K. Kaneko and Y. Ohyama, Fifth Painlevé transcendents which are analytic at the origin, Funkcial. Ekvac. 50 (2007), 187-212. Zbl 1162.34074 MR 2351213

[OK] K. Okamoto and H. Kimura, On particular solutions of the Garnier systems and the hypergeometric functions of several variables, Quart. J. Math. 37 (1986), 61-80. Zbl 0597.35114 MR 0830631

[P] T. Pearcey, The structure of an electromagnetic field in the neighbourhood of a cusp of a caustic, Philos. Mag. 37 (1946), 311-317. MR 0020857

[T] Y. Takei, On a double turning point problem for systems of linear ordinary differential equations, preprint.

[W1] W. Wasow, Asymptotic expansions for ordinary differential equations, Interscience Publ., 1965. Zbl 0133.35301 MR 0203188

[W2] , Linear turning point theory, Appl. Math. Sci. 54, Springer, 1985. Zbl 0558.34049 MR 0771669 